\title{
String diagrams for traced and compact categories are oriented 1-cobordisms
}

\author{
David I. Spivak* Patrick Schultz* \\ Massachusetts Institute of Technology, Cambridge, MA 02139 \\ Dylan Rupel ${ }^{\dagger, \ddagger}$ \\ Northeastern University, Boston, MA 02115
}

\begin{abstract}
We give an alternate conception of string diagrams as labeled 1-dimensional oriented cobordisms, the operad of which we denote by $\mathbf{C o b} / \mathcal{O}$, where $\mathcal{O}$ is the set of string labels. The axioms of traced (symmetric monoidal) categories are fully encoded by $\mathbf{C o b}_{/ \mathcal{O}}$ in the sense that there is an equivalence between $\mathbf{C o b}_{/ \mathcal{O}}$-algebras, for varying $\mathcal{O}$, and traced categories with varying object set. The same holds for compact (closed) categories, the difference being in terms of variance in $\mathcal{O}$. As a consequence of our main theorem, we give a characterization of the 2-category of traced categories solely in terms of those of monoidal and compact categories, without any reference to the usual structures or axioms of traced categories. In an appendix we offer a complete proof of the well-known relationship between the 2-category of monoidal categories with strong monoidal functors and the 2-category of monoidal categories whose object set is free with strict functors; similarly for traced and compact categories.
\end{abstract}

Keywords: Traced monoidal categories, compact closed categories, monoidal categories, lax functors, equipments, operads, factorization systems.

\section{Contents}

1 Introduction $\quad 2$

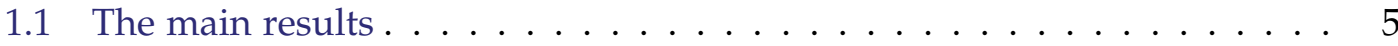

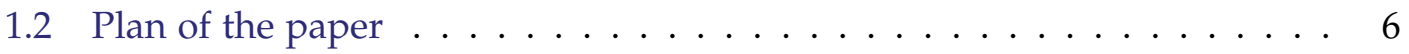

2 Background on equipments $\quad 7$

2.1 Equipments . . . . . . . . . . . . . . . . . 7

2.2 Monoids and bimodules . . . . . . . . . . . . . . . . . . . 12

2.3 Exact equipments and bo, ff factorization . . . . . . . . . . . . 14

*Supported by AFOSR grant FA9550-14-1-0031, ONR grant N000141310260, and NASA grant NNL14AA05C.

†Corresponding author

${ }^{\ddagger}$ Present address: University of Notre Dame, Notre Dame, IN 46556

Email addresses: dspivak@math.mit.edu,schultzp@mit.edu,drupel@nd.edu

(C) 2016. This manuscript version is made available under the Elsevier user license http://www.elsevier.com/open-access/userlicense/1.0/ 
3 Equipments of monoidal profunctors $\quad 21$

3.1 Monoidal, Compact, and Traced Categories . . . . . . . . . . . . . . . 21

3.2 Monoidal profunctors . . . . . . . . . . . . . . . 23

$3.3 \mathbb{M n P r o f}, \mathbb{C}$ pProf, and TrProf are exact $\ldots \ldots \ldots \ldots \ldots$

3.4 Special properties of $\mathbb{C}$ pProf $\ldots \ldots \ldots \ldots \ldots$

3.5 Objectwise-freeness . . . . . . . . . . . . . . . . . . . . . . . . . . . . .

3.6 A traceless characterization of $\widetilde{\mathcal{T r C a t}^{2}} \ldots \ldots \ldots . \ldots . \ldots 40$

$\begin{array}{ll}\text { A Appendix } & 41\end{array}$

A.1 Arrow objects and mapping path objects . . . . . . . . . . . . . . 41

A.2 Strict vs. strong morphisms . . . . . . . . . . . . . . . 46

A.3 Objectwise-free monoidal, traced, and compact categories . . . . . . 51

Bibliography

\section{Introduction}

Traced (symmetric monoidal) categories have been used to model processes with feedback [1] or operators with fixed points [17]. A graphical calculus for traced categories was developed by Joyal, Street, and Verity [12] in which string diagrams of the form

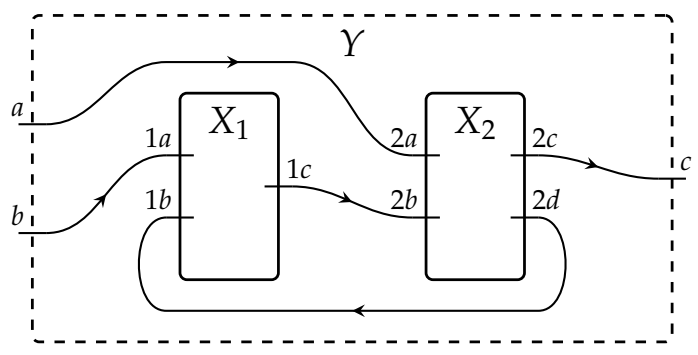

represent compositions in a traced category $\mathscr{T}$. That is, new morphisms are constructed from old by specifying which outputs will be fed back into which inputs. These are related to Penrose diagrams in Vect and the word traced originates in this vector space terminology.

The string diagrams of [12] typically do not explicitly include the outer box $Y$. If we include it, as in (1), the resulting wiring diagram can be given a seemingly new interpretation: it represents a 1-dimensional cobordism between oriented 0-manifolds. Indeed, the objects in Cob are signed sets $X=\left(X^{-}, X^{+}\right)$, each of which can be drawn as a box with input wires $X^{-}$entering on the left and output wires $X^{+}$exiting on the right.

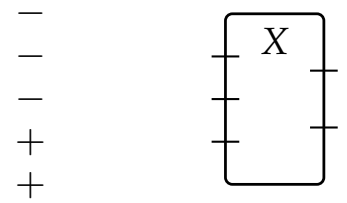

Moreover, the wiring diagram itself in which boxes $X_{1}, \ldots, X_{n}$ are wired together inside a larger box $Y$ can be interpreted as an oriented cobordism from $X_{1} \sqcup \cdots \sqcup X_{n}$ to $Y$. In 
fact, this is more appropriately interpreted as a morphism in the (colored) operad Cob underlying the symmetric monoidal category of oriented 1-cobordisms. The following shows the two approaches to drawing a 2-ary morphism $X_{1}, X_{2} \rightarrow Y$ in Cob:
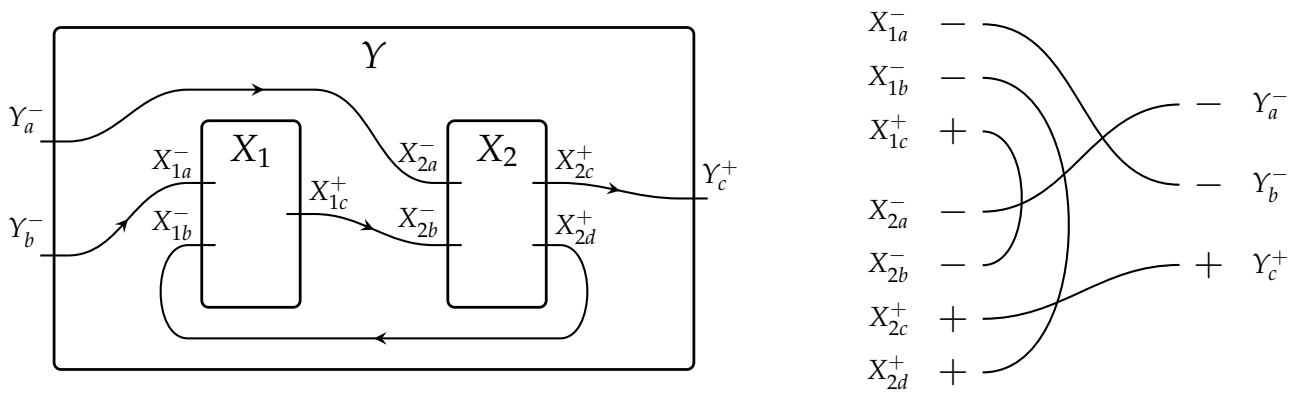

There is actually a bit more data in a string (or wiring) diagram for a traced category $\mathscr{T}$ than in a cobordism. Namely, each input and output of a box must be labeled by an object of $\mathscr{T}$ and the wires connecting boxes must respect the labels (e.g. in (1) objects $1 c$ and $2 b$ must be equal). We will thus consider the operad $\mathbf{C o b}_{/ \mathcal{O}}$ of oriented 1 -dimensional cobordisms over a fixed set of labels $\mathcal{O}$. We also write $\mathbf{C o b} / \mathcal{O}$ to denote the corresponding symmetric monoidal category.

In the table below, we record these two interpretations of a string diagram. Note the "degree shift" between the second and third columns.

\begin{tabular}{lll}
\hline & \multicolumn{2}{c}{ Interpretations of string diagrams } \\
\hline String diagram & Traced category $\mathscr{T}$ & Cob $_{/ \mathcal{O}}$ \\
\hline Wire label set, $\mathcal{O}$ & Objects, $\mathcal{O}:=\mathrm{Ob}(\mathscr{T})$ & Label set, $\mathcal{O}$ \\
Boxes, e.g. $€$ & Morphisms in $\mathscr{T}$ & Objects (oriented 0-mfds over $\mathcal{O}$ ) \\
String diagrams & Compositions in $\mathscr{T}$ & Morphisms (cobordisms over $\mathcal{O}$ ) \\
Nesting & Axioms of traced cats & Composition (of cobordisms) \\
\hline
\end{tabular}

In the last row above, each of the seven axioms of traced categories is vacuous from the cobordism perspective in the sense that both sides of the equation correspond to the same cobordism (up to diffeomorphism). For example, the axiom of superposition reads:

$$
\operatorname{Tr}_{X, Y}^{U}[f] \otimes g=\operatorname{Tr}_{X \otimes W, Y \otimes Z}^{U}[f \otimes g]
$$

for every $f: U \otimes X \rightarrow U \otimes Y$ and $g: W \rightarrow Z$, or diagramatically:
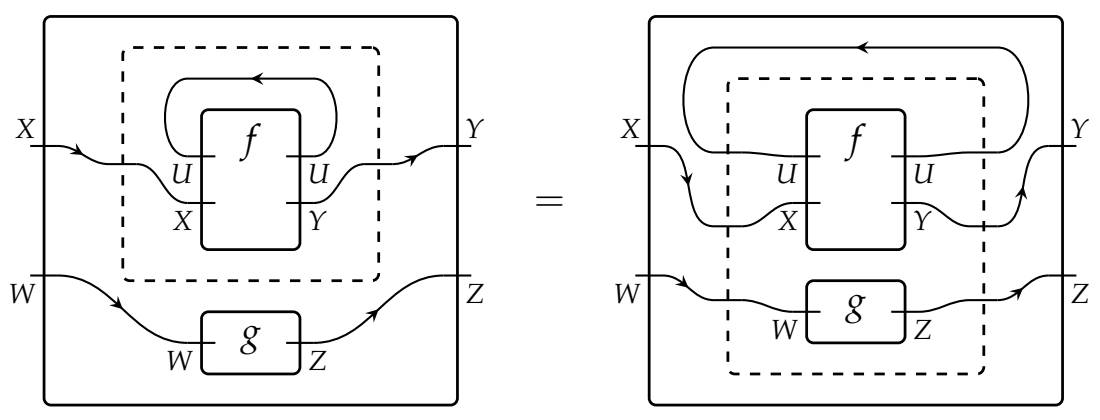
To make precise the relationship between these interpretations of string diagrams, we fix the set $\mathcal{O}$ of labels. Let TrCat denote the 1-category of traced categories and traced strict monoidal functors. Write $\operatorname{TrCat}_{\mathcal{O}}$ for the subcategory consisting of those traced categories $\mathscr{T}$ for which the monoid of objects is free on the set $\mathcal{O}$, with identity-on-objects functors $\mathscr{T} \rightarrow \mathscr{T}^{\prime}$ between them.

Theorem $\mathbf{0 .}$ There is an equivalence of 1-categories

$$
\mathrm{Cob}_{/ \mathcal{O}}-\mathrm{Alg} \simeq \operatorname{TrCat}_{\mathcal{O}}
$$

where, given any monoidal category $\mathcal{M}$, we denote by $\mathcal{M}-\mathbf{A l g}:=\operatorname{Lax}(\mathcal{M}$, Set $)$ the category of lax functors $\mathcal{M} \rightarrow$ Set and monoidal natural transformations.

To build intuition for this statement note that the same data are required, and the same conditions are satisfied, whether one is specifying a lax functor $P \in \mathbf{C o b}_{/ \mathcal{O}^{-}}-\mathbf{A l g}$ or a traced category $\mathscr{T} \in \operatorname{TrCat}_{\mathcal{O}}$ with objects freely generated by the set $\mathcal{O}$. First, for each box $X=\left(X^{-}, X^{+}\right)$that might appear in a string diagram, both $P: \mathbf{C o b}_{/ \mathcal{O}} \rightarrow$ Set and $\mathscr{T}$ require a set, $P(X)$ and $\operatorname{Hom}_{\mathscr{T}}\left(X^{-}, X^{+}\right)$, respectively. Second, for each string diagram, both $P$ and $\mathscr{T}$ require a function: an action on morphisms in the case of $P$ and a formula for performing the required compositions, tensors, and traces in the case of $\mathscr{T}$. The condition that $P$ is functorial corresponds to the fact that $\mathscr{T}$ satisfies the axioms of traced categories.

We will briefly specify how to construct a lax functor $P$ from a traced category $(\mathscr{T}, \otimes, I, \operatorname{Tr})$ whose objects are freely generated by $\mathcal{O}$. In what follows, we abuse notation slightly: given a relative set $\iota: Z \rightarrow \mathcal{O}$ we will use the same symbol $Z$ to denote the tensor $\bigotimes_{z \in Z} \iota(z)$ in $\mathscr{T}$. For an oriented 0-manifold $X=X^{-} \sqcup X^{+}$over $\mathcal{O}$, put $P(X):=$ $\operatorname{Hom}_{\mathscr{T}}\left(X^{-}, X^{+}\right)$. Given a cobordism $\Phi: X \rightarrow Y$, we need a function $P(\Phi): P(X) \rightarrow$ $P(Y)$. To specify it, note that for any cobordism $\Phi$ there exist $A, B, C, D, E \in \mathrm{Ob}(\mathscr{T})$ such that $X^{-} \cong C \otimes A, X^{+} \cong C \otimes B, Y^{-} \cong A \otimes D, Y^{+} \cong B \otimes D$, and $E$ is the set of floating loops in $\Phi$; thus $\Phi$ is essentially equivalent to the cobordism shown on the left side of (3).
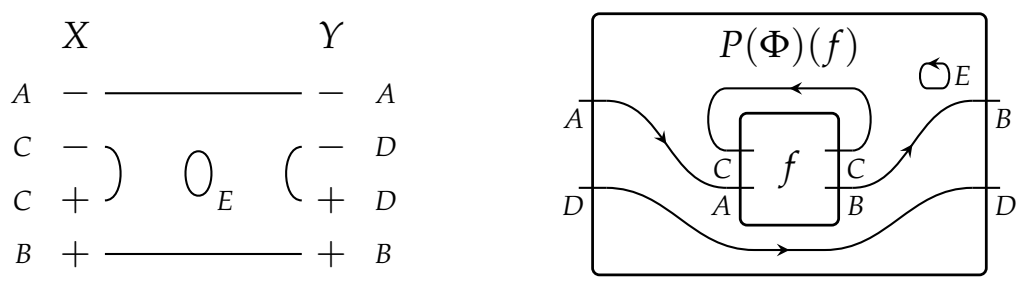

With the above notation, for $f \in P(X)$ we can follow the string diagram (right side of (3)) and define

$$
P(\Phi)(f):=\operatorname{Tr}_{A, B}^{C}[f] \otimes D \otimes \operatorname{Tr}_{I, I}^{E}[E],
$$

where we abuse notation and write $D$ and $E$ for the identity maps on these objects. One may easily check, using each axiom of the trace [12] in an essential way, that (4) defines an algebra over $\mathbf{C o b}_{/ \mathcal{O}}$. We will not prove Theorem 0 directly as indicated here; to specify our proof strategy we must introduce more notation. 


\subsection{The main results}

The equivalence (2) has two significant conceptual drawbacks. First, the object set of the traced category $\mathscr{T}$ is fixed; second, $\mathscr{T}$ is assumed to be freely generated by some set under tensor products and functors are assumed to be strict. We refer to this latter condition using the term objectwise-free. Much of the work in this paper goes towards relaxing these two conditions; for now we continue to assume objectwise-freeness.

To overcome the use of a fixed object set, we first explain what kind of object variance is appropriate. There is an adjunction

$$
\text { Set } \underset{\mathrm{U}_{\mathrm{T}}}{\stackrel{\mathrm{F}_{\mathrm{T}}}{\rightleftarrows}} \operatorname{TrCat}
$$

inducing a monad $\mathbf{T}_{\mathrm{T}}$ on Set, which is in fact isomorphic to the free monoid monad. This induces a canonical functor from TrCat to the Eilenberg-Moore category Set $^{\mathbf{T}_{\top}}$ of this monad, sending each traced category to its underlying monoid of objects. We define TrFrObCat $\subset$ TrCat to be the full subcategory spanned by the objectwise-free traced categories. In other words, TrFrObCat is the pullback of TrCat $\rightarrow$ Set $^{\mathbf{T}_{\mathrm{T}}}$ along the fully-faithful functor Set $_{\mathbf{T}_{\mathrm{T}}} \rightarrow$ Set $^{\mathbf{T}_{\mathrm{T}}}$ from the Kleisli category to the Eilenberg-Moore category, or equivalently along the inclusion of free monoids into all monoids.

The compact category $\mathbf{C o b}_{/ \mathcal{O}}$ clearly varies functorially in $\mathcal{O} \in$ Set, but it is not much harder to see that it is also functorial in $\mathcal{O} \in \mathbf{S e t}_{\mathbf{T}_{\mathbf{T}}}$. This gives rise to a functor

$$
\left(\operatorname{Cob}_{/ \bullet}\right): \operatorname{Set}_{\mathbf{T}_{\mathrm{T}}} \rightarrow \text { CpCat }
$$

to the category CpCat of compact categories and strict functors, sending $\mathcal{O}$ to $\mathrm{Cob}_{/ \mathcal{O}}$, the free compact category on $\mathcal{O}$ (e.g. see $[13,2])$. We can compose this with $\operatorname{Lax}(-$, Set $)$ to obtain a functor which we denote

$$
\left(\mathrm{Cob}_{/ \bullet}\right)-\mathrm{Alg}: \operatorname{Set}_{\mathbf{T}_{\mathrm{T}}}^{\mathrm{op}} \longrightarrow \text { Cat. }
$$

By applying the Grothendieck construction (denoted by $\int$ here) to (6), we obtain a fibration for which the fiber over a set $\mathcal{O}$ is equivalent (by Theorem 0 ) to $\operatorname{TrCat}_{\mathcal{O}}$.

Theorem A. There is an equivalence of 1-categories

$$
\int^{\mathcal{O} \in \operatorname{Set}_{\mathrm{T}_{\mathrm{T}}}}\left(\mathrm{Cob}_{/ \mathcal{O}}\right)-\mathrm{Alg} \stackrel{\simeq}{\rightarrow} \text { TrFrObCat. }
$$

This result, together with an analogous statement for compact categories, is proven in Section 3.5.

The fact that the traced categories appearing in Theorem A are assumed objectwisefree and the functors between them are strict is the second of two drawbacks mentioned above. To address it, we prove that the 2-category $\mathcal{T}$ rFrObCat, of objectwise-free traced categories and strict functors, is biequivalent to that of arbitrary traced categories and strong functors; see Corollary A.3.2. This result seems to be well-known to experts but is difficult to find in the literature. 
In the course of proving Theorem A, we will also establish generalizations characterizing lax functors out of arbitrary compact categories. In order to state this characterization, we prove (Theorem 2.3.15 and Proposition 3.3.2) that the well-known (bo, ff) factorization system of Cat restricts to a factorization system on TrCat; more precisely the left class consists of bijective-on-objects functors and the right class consists of fully faithful functors.

Write $\operatorname{TrCat}^{\text {bo }}$ for the full subcategory of the arrow category $\operatorname{TrCat}^{\rightarrow}$ spanned by the bijective-on-objects functors. The existence of the factorization system implies that the domain functor

$$
\text { dom: } \operatorname{TrCat}^{\text {bo }} \rightarrow \text { TrCat }
$$

is a fibration. For a fixed traced category $\mathscr{T}$, the fiber $\operatorname{TrCat}_{\mathscr{T} /}^{\text {bo }}:=\operatorname{dom}^{-1}(\mathscr{T})$ is the category of strict monoidal, bijective-on-objects functors from $\mathscr{T}$ to another traced category, with the evident commutative triangles as morphisms. Note that (with $\mathbf{F}_{\mathbf{T}}$ as in (5)) we have an isomorphism $\operatorname{TrCat}_{\mathcal{O}} \cong \operatorname{TrCat}_{\left(\mathbf{F}_{\mathrm{T}} \mathcal{O}\right) /}^{\mathrm{bo}}$.

Recall from [12] that traced categories can be thought of as full subcategories of compact categories: the Int construction applied to a traced category $\mathscr{T}$ builds the smallest compact category $\operatorname{Int}(\mathscr{T})$ of which $\mathscr{T}$ is a monoidal subcategory, we refer the reader to [12] for more details. Generalizing (2), we can give a complete characterization of lax functors out of such compact categories: for a fixed traced category $\mathscr{T}$ there is an equivalence of categories

$$
\operatorname{Lax}(\operatorname{Int}(\mathscr{T}), \text { Set }) \simeq \operatorname{TrCat}_{\mathscr{T} /}^{\text {bo }}
$$

In Section 3.3 we show that these equivalences glue together to form an equivalence of fibrations:

Theorem B. There is an equivalence of fibrations

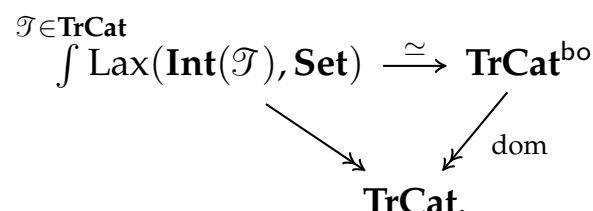

Theorem A will follow from this by restricting to just those $\mathscr{T} \in$ TrCat which are free on a set $\mathcal{O}$.

Our main tool in proving this result will be the 2-categorical notion of (proarrow) equipments, which we recall in Section 2. We will introduce what appears to be a new definition of monoidal profunctors, and the equipment thereof, in Section 3.

\subsection{Plan of the paper}

Section 2.1 reviews the definition of an equipment (or framed bicategory [20]), while Section 2.2 recalls monoids and bimodules in an equipment. We define exact equipments in Section 2.3, which are central to our proof strategy, and which we believe to be of independent interest. The material of this section is original, though some of it appeared in the earlier unpublished [19]. 
Section 3.1 briefly reviews monoidal, traced, and compact categories. In Section 3.2 we introduce our main objects of study, namely various equipments of monoidal profunctors (MnProf, TrProf, and $\mathbb{C}$ pProf), which we prove are exact in Section 3.3. Section 3.4 is devoted to the special properties of $\mathbb{C}$ pProf which are at the core of our results-indeed one might view the rest of the paper as a formal wrapper around the results in that section-and concludes with the proof of Theorem B. In Section 3.5 we deal with the issue of objectwise-freeness before proving Theorem A. Section 3.6 briefly records an interesting byproduct of the theory developed for the proofs of the main theorems: a (we believe) new characterization, up to biequivalence, of the 2-category of traced monoidal categories which makes no mention of a trace operation.

The appendix-Sections A.1, A.2, and A.3-contains material that is not essential for establishing the main results of the paper. The purpose of the appendix is to prove the biequivalence between the 2-category $\widetilde{\mathrm{MnCat}}$ of monoidal categories with arbitrary object set and strong functors, on the one hand, and the 2-category MnFrObCat of monoidal categories with free object set and strict functors, on the other. We do the same for traced and compact categories, all in Corollary A.3.2.

\section{Acknowledgments}

Thanks go to Steve Awodey and Ed Morehouse for suggesting we formally connect the operad-algebra picture in [18] to string diagrams in traced categories. We also thank Mike Shulman for many useful conversations, and Tobias Fritz, Justin Hilburn, Dmitry Vagner, and Christina Vasilakopoulou for helpful comments on drafts of this paper. Finally, we thank the anonymous referee whose suggestions were extremely helpful in making this paper more readable.

\section{Background on equipments}

One of the main technical tools used in our proofs of the main theorems is monoidal profunctors, which are profunctors between monoidal categories with extra structure. When studying monoidal/traced/compact categories together with their corresponding functors and natural transformations, a bit of 2-category theory is often useful. Analogously, when studying monoidal profunctors between such structured categories together with their relation to functors and natural transformations, it is helpful to make use of the theory of equipments.

In this section we review the material on equipments that will be needed in the sequel.

\subsection{Equipments}

A double category is a 2-category-like structure involving horizontal and vertical arrows, as well as 2-cells. An equipment (sometimes called a proarrow equipment or framed bicategory) is a double category satisfying a certain fibrancy condition. In this 
section, we will spell this out and give two relevant examples. An excellent reference is Shulman's paper [20]; see also [21] and [22].

Definition 2.1.1. A double category ${ }^{1} \mathrm{D}$ consists of the following data:

- A category $\mathbb{D}_{0}$, which we refer to as the vertical category of $\mathbb{D}$. For any two objects $c, d \in \mathbb{D}_{0}$, we will write $\mathbb{D}_{0}(c, d)$ for the set of vertical arrows from $c$ to $d$. We refer to objects of $\mathbb{D}_{0}$ as objects of $\mathbb{D}$.

- A category $\mathbb{D}_{1}$, equipped with two functors $L, R: \mathbb{D}_{1} \rightarrow \mathbb{D}_{0}$, called the left frame and right frame functors. Given an object $M \in \mathrm{Ob}\left(\mathbb{D}_{1}\right)$ with $c=\mathrm{L}(M)$ and $c^{\prime}=\mathrm{R}(M)$, we say that $M$ is a proarrow (or horizontal arrow) from $c$ to $c^{\prime}$ and write $M: c \rightarrow c^{\prime}$. A morphism $\phi: M \rightarrow N$ in $\mathbb{D}_{1}$ is called a 2-cell, and is drawn as follows, where $f=\mathrm{L}(\phi)$ and $f^{\prime}=\mathrm{R}(\phi)$ :

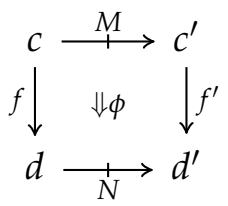

- A unit functor $U: \mathbb{D}_{0} \rightarrow \mathbb{D}_{1}$, which is a strict section of both $L$ and $R$, i.e. $L \circ U=$ $\operatorname{id}_{\mathbb{D}_{0}}=\mathrm{R} \circ \mathrm{U}$. We will often abuse notation by writing $c$ for the unit proarrow $\mathrm{U}(c): c \rightarrow c$, and similarly for vertical arrows.

- A functor $\odot: \mathbb{D}_{1} \times \mathbb{D}_{0} \mathbb{D}_{1} \rightarrow \mathbb{D}_{1}$, called horizontal composition, which is weakly associative and unital in the sense that there are coherent unitor and associator isomorphisms. See [20] for more details.

Given a double category $\mathbb{D}$ there is a strict 2-category called the vertical 2-category, denoted $\mathcal{V} \operatorname{ert}(\mathbb{D})$, whose underlying 1-category is $\mathbb{D}_{0}$ and whose 2-morphisms $f \Rightarrow f^{\prime}$ are defined to be 2-cells (7) where $M=\mathrm{U}(c)$ and $N=\mathrm{U}(d)$ are unit proarrows. There is also a horizontal bicategory, denoted $\mathcal{H} \operatorname{cor}(\mathbb{D})$, whose objects and 1-cells are the objects and horizontal arrows of $\mathbb{D}$, and whose 2-cells are the 2-cells of $\mathbb{D}$ of the form (7) such that $f=\mathrm{id}_{c}$ and $f^{\prime}=\mathrm{id}_{c^{\prime}}$.

A strong double functor $F: \mathbb{C} \rightarrow \mathbb{D}$ consists of functors $F_{0}: \mathbb{C}_{0} \rightarrow \mathbb{D}_{0}$ and $F_{1}: \mathbb{C}_{1} \rightarrow$ $\mathbb{D}_{1}$ commuting with the frames $L, R$, which preserve the unit $U$ and the horizontal composition $\odot$ up to coherent isomorphism.

Recall that a fibration of categories $p: \mathscr{E} \rightarrow \mathscr{B}$ is a functor with a lifting property: for every $f: b^{\prime} \rightarrow b$ in $\mathscr{B}$ and object $e \in \mathscr{E}$ with $p(e)=b$, there exists $e^{\prime} \rightarrow e$ over $f$ that is cartesian, i.e. universal in an appropriate sense. We denote fibrations of 1-categories using two-headed arrows $\rightarrow$.

\footnotetext{
${ }^{1}$ We will use many flavors of category in this paper, and we attempt to use different fonts to distinguish between them. We denote named 1-categories, monoidal categories, and operads using bold roman letters, e.g. Cob, and unnamed 1-categories with script, e.g. $\mathscr{C}$. For named 2-categories or bicategories we do almost the same, but change the font of the first letter to calligraphic, such as $\mathcal{T}$ rCat; for unnamed 2-categories we use (unbold) calligraphic, e.g. D. Finally, for double categories we make the first letter blackboard bold, whether named (e.g., Prof) or unnamed (e.g. D). A minor exception occurs almost immediately, however: two 1-categories appear as part of the structure of a double category $\mathbb{D}$, and we denote them as $\mathbb{D}_{0}, \mathbb{D}_{1}$ rather than using script font.
} 
Definition 2.1.2. An equipment is a double category $\mathbb{D}$ in which the frame functor

$$
(\mathrm{L}, \mathrm{R}): \mathbb{D}_{1} \rightarrow \mathbb{D}_{0} \times \mathbb{D}_{0}
$$

is a fibration. Given vertical morphisms $f: c \rightarrow d$ and $f^{\prime}: c^{\prime} \rightarrow d^{\prime}$ together with a proarrow $N: d \rightarrow d^{\prime}$, a cartesian morphism $M \rightarrow N$ in $\mathbb{D}_{1}$ over $\left(f, f^{\prime}\right)$ is a 2-cell

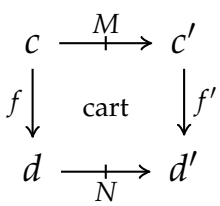

which we call a cartesian 2-cell. We refer to $M$ as the restriction of $N$ along $f$ and $f^{\prime}$, written $M=N\left(f, f^{\prime}\right)$.

For any vertical morphism $f: c \rightarrow d$ in an equipment $\mathbb{D}$, there are two canonical proarrows $\widehat{f}: c \rightarrow d$ and $\breve{f}: d \rightarrow c$, called respectively the companion and the conjoint of $f$, defined by restriction:

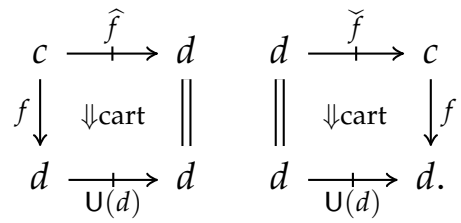

In [20], it is shown that all restrictions can be obtained by composing with companions and conjoints. In particular, $N\left(f, f^{\prime}\right) \cong \widehat{f} \odot N \odot \check{f}^{\prime}$ for any proarrow $N$. Moreover, $\widehat{f}$ and $\breve{f}$ form an adjunction in $\mathcal{H} \operatorname{Cor}(\mathbb{D})$; we denote the unit and counit by:

$$
\eta_{f}: \mathrm{U}(c) \rightarrow \widehat{f} \odot \check{f} \quad \text { and } \quad \epsilon_{f}: \check{f} \odot \widehat{f} \rightarrow \mathrm{U}(d)
$$

Recall that a pseudo-pullback of a cospan $A_{1} \stackrel{f_{1}}{\longrightarrow} A \stackrel{f_{2}}{\longleftarrow} A_{2}$ in a 2-category $\mathcal{C}$ is a diagram

$$
\begin{aligned}
& X \stackrel{g_{1}}{\longrightarrow} A_{2} \\
& g_{2} \downarrow \stackrel{\alpha}{\cong} \downarrow f_{2} \\
& A_{1} \underset{f_{1}}{\longrightarrow} A
\end{aligned}
$$

where the tuple $\left(X, g_{1}, g_{2}, \alpha\right)$ is universal, up to equivalence, for data of that shape. Although this definition makes sense for any 2-category $\mathcal{C}$, we will use it only in the special case described in the next paragraph.

Let $\mathcal{C}=\mathcal{C}$ at, the 2 -category of small categories. Suppose $f_{2}$ is a fibration and that the pullback square (10) strictly commutes, i.e. that $\alpha$ is the identity. It is a standard fact that a strict pullback of a fibration along an arbitrary functor is a fibration. Moreover, this strict pullback is also a pseudo-pullback. The upshot is that in (10), $g_{2}$ is a pseudopullback if and only if for any strict pullback $g_{2}^{\prime}$ of $f_{2}$ along $f_{1}$, the induced map $g_{2} \rightarrow g_{2}^{\prime}$ is an equivalence of fibrations. 
Definition 2.1.3. An equipment functor is a strong double functor between equipments (see Definition 2.1.1).

An equipment functor $F: \mathbb{C} \rightarrow \mathbb{D}$ is called a local equivalence if the following (strictly commuting) square is a pseudo-pullback of categories:

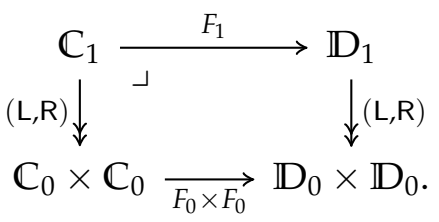

If moreover $F_{0}: \mathbb{C}_{0} \rightarrow \mathbb{D}_{0}$ is fully faithful, we say that $F$ is a fully faithful local equivalence.

Remark 2.1.4. As discussed above, if the square (11) is a strict pullback, it will be a pseudo-pullback, and hence a local equivalence. Any local equivalence can thus be replaced by an equivalent strict pullback. We will use this fact often; see Definition 2.1.5.

Also note that the frame fibration for $\mathbb{C}$ is equivalent to a functor $\mathbb{C}_{0} \times \mathbb{C}_{0} \rightarrow$ Cat, sending $(c, d)$ to $\mathcal{H o r}(\mathbb{C})(c, d)$ and similarly for $\mathbb{D}$. In this language, $F$ is a local equivalence if and only if the induced functors $\mathcal{H} \operatorname{or}(\mathbb{C})(c, d) \rightarrow \mathcal{H} \operatorname{or}(\mathbb{D})\left(F_{0}(c), F_{0}(d)\right)$ are equivalences of categories for every pair of objects $(c, d)$. The square (11) is a strict pullback precisely when these are isomorphisms of categories.

Definition 2.1.5. Let $\mathbb{D}$ be a double category and $F_{0}: \mathbb{C}_{0} \rightarrow \mathbb{D}_{0}$ be a functor. A strict pullback of the form (11) defines a double category with vertical category $\mathbb{C}_{0}$, which we denote $F_{0}^{*}(\mathbb{D})$.

If $\mathbb{D}$ is an equipment, $F_{0}^{*}(\mathbb{D})$ will be one as well since fibrations are stable under pullback. In this case we call $F_{0}^{*}(\mathbb{D})$ the equipment induced by $F_{0}$. By Remark 2.1.4, the induced equipment functor $F_{0}^{*}(\mathbb{D}) \rightarrow \mathbb{D}$ is a local equivalence.

The structured categories of interest in this paper can be conveniently organized using the notion of equipments. The prototype for all of these equipments is that of categories, functors, and profunctors as in the following example.

Example 2.1.6. The equipment $\mathbb{P r o f}$ is a double category whose vertical category $\operatorname{Prof}_{0}=$ Cat is the category of small 1 -categories and functors. Given categories $\mathscr{C}, \mathscr{C}^{\prime} \in \operatorname{Prof}_{0}$, a proarrow

$$
\mathscr{C} \stackrel{M}{\longrightarrow} \mathscr{C}^{\prime}
$$

in $\operatorname{Prof}_{1}$ is a profunctor, i.e. a functor $M: \mathscr{C}^{\mathrm{op}} \times \mathscr{C}^{\prime} \rightarrow$ Set. The left and right frame functors are given by $\mathrm{L}(M)=\mathscr{C}$ and $\mathrm{R}(M)=\mathscr{C}^{\prime}$. A 2-cell $\phi$ in Prof, as to the left, denotes a natural transformation, as to the right, in (12):
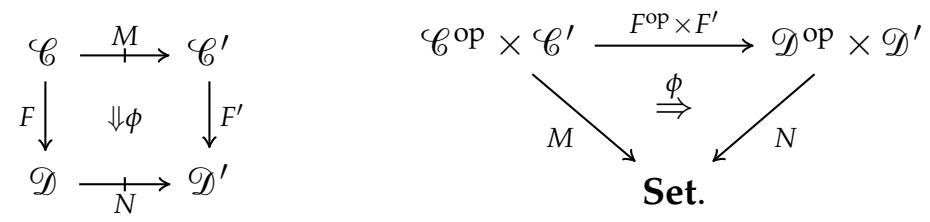
The unit functor U: Cat $\rightarrow \mathbb{P r o f}_{1}$ sends a category $\mathscr{C}$ to the hom profunctor Hom $_{\mathscr{C}}: \mathscr{C}^{\text {op }} \times$ $\mathscr{C} \rightarrow$ Set. Given two profunctors

$$
\mathscr{C} \stackrel{M}{\longrightarrow} \mathscr{D} \stackrel{N}{\longrightarrow} \mathscr{E},
$$

define the horizontal composition $M \odot N$ on objects $c \in \mathscr{C}$ and $e \in \mathscr{E}$ as the reflexive coequalizer of the diagram

$$
\coprod_{d_{1}, d_{2} \in \mathscr{D}} M\left(c, d_{1}\right) \times \mathscr{D}\left(d_{1}, d_{2}\right) \times N\left(d_{2}, e\right) \rightleftarrows \coprod_{d \in \mathscr{D}} M(c, d) \times N(d, e)
$$

where the two rightward maps are given by the right and left actions of $\mathscr{D}$ on $M$ and $N$ respectively, and the splitting is given by $\operatorname{id}_{d} \in \mathscr{D}(d, d)$. Given a profunctor $M: \mathscr{C} \rightarrow \mathscr{D}$ there are canonical isomorphisms Hom $_{\mathscr{C}} \odot M \cong M \cong M \odot$ Hom $_{\mathscr{D}}$ which can be viewed as giving an action of $\operatorname{Hom}_{\mathscr{C}}$ and of $\mathrm{Hom}_{\mathscr{D}}$ on $M$, from the left and right respectively.

At this point we have given Prof the structure of a double category. To see that $\mathbb{P}$ rof is an equipment, note that from a pair of functors $F: \mathscr{C} \rightarrow \mathscr{D}, F^{\prime}: \mathscr{C}^{\prime} \rightarrow \mathscr{D}^{\prime}$ and a profunctor $N: \mathscr{D} \rightarrow \mathscr{D}^{\prime}$ we may form the composite

$$
\mathscr{C} \text { op } \times \mathscr{C}^{\prime} \stackrel{F^{\mathrm{op}} \times F^{\prime}}{\longrightarrow} \mathscr{D}^{\mathrm{op}} \times \mathscr{D}^{\prime} \stackrel{N}{\longrightarrow} \text { Set, }
$$

denoted $N\left(F, F^{\prime}\right): \mathscr{C} \rightarrow \mathscr{C}^{\prime}$, such that

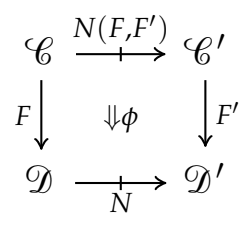

is a cartesian 2-cell. A simple Yoneda lemma argument yields $\operatorname{Vert}(\mathbb{P r o f}) \simeq$ Cat.

Remark 2.1.7. There is a strong analogy relating profunctors between categories with bimodules between rings. Besides being a useful source of intuition, we can also exploit this analogy to provide a convenient notation for working with profunctors.

If $M: \mathscr{C}^{\text {op }} \times \mathscr{D} \rightarrow$ Set is a profunctor, then for any element $m \in M(c, d)$ and morphisms $f: c^{\prime} \rightarrow c$ and $g: d \rightarrow d^{\prime}$, we can write $g \cdot m \in M\left(c, d^{\prime}\right)$ and $m \cdot f \in M\left(c^{\prime}, d\right)$ for the elements $M(\mathrm{id}, g)(m)$ and $M(f, \mathrm{id})(m)$ respectively. Thus we think of the functoriality of $M$ as providing left and right actions of $\mathscr{D}$ and $\mathscr{C}$ on the elements of M. The equations $(g \cdot m) \cdot f=g \cdot(m \cdot f), g^{\prime} \cdot(g \cdot m)=\left(g^{\prime} \circ g\right) \cdot m$, and $(m \cdot f) \cdot f^{\prime}=$ $m \cdot\left(f \circ f^{\prime}\right)$ clearly hold whenever they make sense.

The reflexive coequalizer (13) can be easily expressed in this notation: the elements of $(M \odot N)(c, e)$ are pairs $m \otimes n$ of elements $m \in M(c, d)$ and $n \in N(d, e)$ for some $d \in \mathscr{D}$ modulo the relation $(m \cdot f) \otimes n=m \otimes(f \cdot n)$, for $f \in \mathscr{D}$.

Finally, a 2-cell $\phi$ of the form (12) is function sending elements $m \in M\left(c, c^{\prime}\right)$ to elements $\phi(m) \in N\left(F c, F^{\prime} c^{\prime}\right)$ such that the equation $\phi(g \cdot m \cdot g)=F(g) \cdot \phi(m) \cdot F^{\prime}(f)$ holds whenever it makes sense. 


\subsection{Monoids and bimodules}

In any equipment $\mathbb{D}$, we can consider monads in the horizontal bicategory $\mathcal{H}$ or $(\mathbb{D})$. In [19], a definition of exact equipment was given axiomatizing a class of equipments in which there is a close connection between monads in $\mathcal{H} \operatorname{cor}(\mathbb{D})$ and the objects of $\mathbb{D}$. We will review exact equipments and the results needed here in Section 2.3, but first we collect the necessary definitions and results about monads in $\mathcal{H} \operatorname{Cor}(\mathbb{D})$, which we refer to as monoids in $\mathbb{D}$.

Definition 2.2.1. Denote by $\operatorname{Mon}(\mathbb{D})$ the category of monoids in $\mathbb{D}$. More precisely, the objects are monoids: 4-tuples $\left(c, M, i_{M}, m_{M}\right)$ consisting of an object $c$ of $\mathbb{D}$ and a proarrow $M: c \rightarrow c$, together with unit and multiplication cells
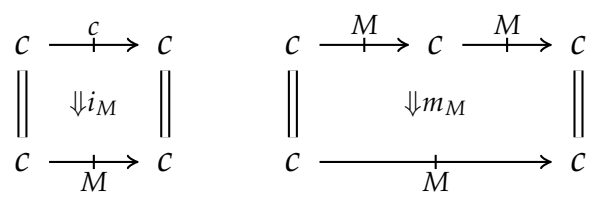

satisfying the evident unit and associativity axioms. The morphisms are monoid homomorphisms: pairs $(f, \vec{f})$ consisting of a vertical arrow $f: c \rightarrow d$ in $\mathbb{D}$ and a cell

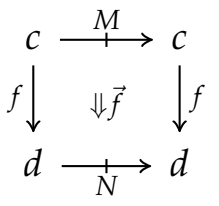

which respects the unit and multiplication cells of $M$ and $N$.

There is an evident forgetful functor $|\cdot|: \operatorname{Mon}(\mathbb{D}) \rightarrow \mathbb{D}_{0}$ sending a monoid $M: c \rightarrow c$ to its underlying object $|M|:=c$. The following result is also in [7].

Lemma 2.2.2. Let $\mathbb{D}$ be an equipment. The forgetful functor $|\cdot|: \operatorname{Mon}(\mathbb{D}) \rightarrow \mathbb{D}_{0}$ is a fibration and there is a morphism of fibrations

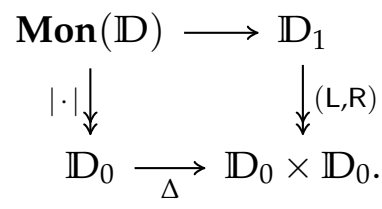

Proof. Let $f: c \rightarrow d$ be a vertical morphism of $\mathbb{D}$ and $N: d \rightarrow d$ a monoid in $\mathbb{D}$. Since the 2-cell defining the restriction of $N$ along $f$ is cartesian, there is an induced monoid structure on $N(f, f)$ which in particular makes this cartesian 2-cell a monoid homomorphism. The result follows.

Lemma 2.2.3. For a local equivalence $F: \mathbb{C} \rightarrow \mathbb{D}$, the induced square

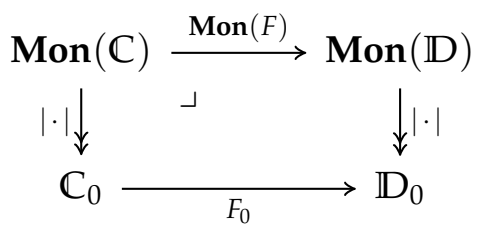


is a pseudo-pullback of categories.

Proof. By Remark 2.1.4, we may assume that the pullback (11) in Definition 2.1.3, realizing $F: \mathbb{C} \rightarrow \mathbb{D}$ as a local equivalence, is strict. It is then straightforward to check directly that the above square is again a strict pullback and hence a pseudo-pullback.

In all our cases of interest, $\operatorname{Mon}(\mathbb{D})$ becomes the vertical part of another equipment. The following is a standard construction; see [20].

Definition 2.2.4. Let $\mathbb{D}$ be an equipment with local reflexive coequalizers, i.e. such that each 1-category $\mathcal{H} \operatorname{or}(\mathbb{D})(c, d)$ has reflexive coequalizers and $\odot$ preserves reflexive coequalizers in each variable. The equipment $\operatorname{Mod}(\mathbb{D})$ of monoids and bimodules is defined as follows:

- The vertical category $\mathbb{M o d}(\mathbb{D})_{0}$ is the category $\operatorname{Mon}(\mathbb{D})$ of monoids in $\mathbb{D}$.

- The proarrows $B: M \rightarrow N$ are bimodules: triples $\left(B, l_{B}, r_{B}\right)$ consisting of a proarrow $B: c \rightarrow d$ in $\mathbb{D}$ and cells
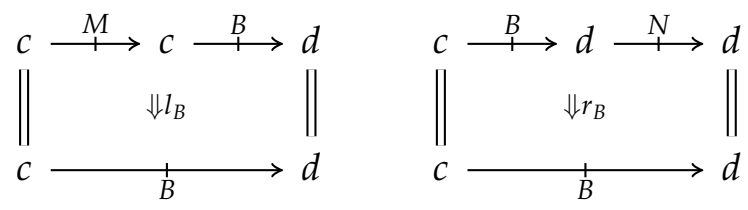

satisfying evident monoid action axioms.

- The horizontal composition $B_{1} \otimes_{M^{\prime}} B_{2}$ of bimodules $B_{1}: M \rightarrow M^{\prime}$ and $B_{2}: M^{\prime} \rightarrow M^{\prime \prime}$ is given by the reflexive coequalizer in $\mathcal{H} \operatorname{or}(\mathbb{D})\left(M, M^{\prime \prime}\right)$

$$
B_{1} \odot M^{\prime} \odot B_{2} \rightleftarrows B_{1} \odot B_{2} \longrightarrow B_{1} \otimes_{M^{\prime}} B_{2}
$$

together with the evident left $M$ and right $M^{\prime \prime}$ actions. Above, the splitting map $B_{1} \odot B_{2} \rightarrow B_{1} \odot M^{\prime} \odot B_{2}$ comes from the unit $i_{M^{\prime}}$ of the monoid.

- The 2-cells are bimodule homomorphisms: cells in $\mathbb{D}$

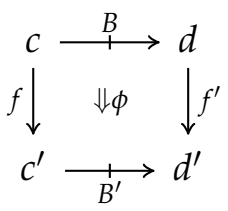

which are compatible with the various left and right monoid actions.

We will write ${ }_{M} \boldsymbol{B i m o d}_{N}:=\mathcal{H} \operatorname{Cor}(\operatorname{Mod}(\mathbb{D}))(M, N)$ to denote the 1-category of $(M, N)$ bimodules and bimodule morphisms.

The forgetful functor $|\cdot|: \operatorname{Mon}(\mathbb{D}) \rightarrow \mathbb{D}_{0}$ extends to a lax equipment functor $|\cdot|: \operatorname{Mod}(\mathbb{D}) \rightarrow \mathbb{D}$. We have not defined lax equipment functors-because we do not use them-and in particular we will we not use the equipment version of $|\cdot|$.

More importantly for our work, there is a local equivalence $U: \mathbb{D} \rightarrow \mathbb{M o d}(\mathbb{D})$ sending $c$ to the unit $c \rightarrow c$ with the trivial monoid structure. If $F: \mathbb{C} \rightarrow \mathbb{D}$ is an equipment functor, then there is an evident equipment functor $\operatorname{Mod}(F): \operatorname{Mod}(\mathbb{C}) \rightarrow$ $\operatorname{Mod}(\mathbb{D})$. In fact, we have the following which is immediate from the definitions. 
Lemma 2.2.5. If $F: \mathbb{C} \rightarrow \mathbb{D}$ is a local equivalence, then so is the induced functor $\mathbb{M o d}(F): \mathbb{M o d}(\mathbb{C}) \rightarrow$ $\operatorname{Mod}(\mathbb{D})$. If $F$ is a fully faithful local equivalence, then so is $\operatorname{Mod}(F)$.

\subsection{Exact equipments and bo, ff factorization}

For many equipments $\mathbb{D}$, monoids in $\mathbb{D}$ are category-like objects where the vertical and horizontal arrows in $\mathbb{M o d}(\mathbb{D})$ are (generalized or structured) functors and profunctors. The canonical example is the equipment of spans of sets whose objects, vertical, and horizontal arrows are sets, functions, and spans respectively. In this case, the equipment of monoids is equivalent to Prof, which may be easily verified directly (or see [16]).

One natural question is thus: what are monoids in equipments like Prof whose objects are already category-like? One might guess they would be higher-dimensional structures like double categories. However, it turns out that in these cases where $\mathbb{D}$ is an equipment of category-like objects, the equipment $\operatorname{Mod}(\mathbb{D})$ of monoids in $\mathbb{D}$ is very closely related to $\mathbb{D}$ itself. In other words, monoids in an equipment are category-like objects, but taking monoids in an already category-like equipment doesn't change much.

The article [19] proposed definitions of regular and exact equipments. For this paper we will only be concerned with exact equipments, which are an attempt to axiomatize the above property of equipments whose objects are category-like. In particular, given any monoid $M$ in an exact equipment, there is a corresponding object called the collapse of $M$ satisfying a certain vertical universal property. This collapse construction has formal similarities to the quotient of an equivalence relation, and it is because of this (informal) analogy that the names regular and exact where chosen. From this perspective, $\operatorname{Mod}(\mathbb{D})$ can be thought of as the exact completion of the equipment $\mathbb{D}$.

In this section we recall the definition of an exact equipment from [19] and review some basic properties. In Section 3.3 we will prove that the equipments of interest are exact. This will allow us to use the collapse construction as a crucial step in the connection between lax monoidal set valued functors-which we view as proarrows in exact equipments-with (traced or compact) monoidal categories-which will be objects of those exact equipments. We will also need to make use of fully-faithful/bijectiveon-objects factorization systems, which are shown in this section to exist in any exact equipment. (In [19] it is shown that this factorization system exists in any regular equipment, as the regular-epi/mono factorization system exists in any regular category).

Definition 2.3.1. Let $M: c \rightarrow c$ be a monoid in an equipment $\mathbb{D}$. An embedding of $M$ into an object $x \in \mathbb{D}_{0}$ is a monoid homomorphism $(f, \vec{f})$ from $M$ to the trivial monoid on $x$ :

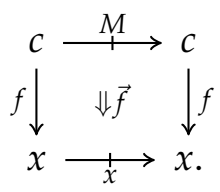

We will sometimes write an embedding as $(f, \vec{f}):(c, M) \rightarrow x$, or even just $f: M \rightarrow x$ when clear from context. We will write $\operatorname{Emb}(M, x)$ for the set of embeddings from $M$ to $x$. This defines a functor $\operatorname{Emb}: \operatorname{Mon}(\mathbb{D})^{\mathrm{op}} \times \mathbb{D}_{0} \rightarrow$ Set. 
Lemma 2.3.2. Suppose that $F: \mathbb{C} \rightarrow \mathbb{D}$ is a local equivalence induced by $F_{0}: \mathbb{C}_{0} \rightarrow \mathbb{D}_{0}$. Suppose $M \in \operatorname{Mon}(\mathbb{C})$ is a monoid and $x \in \mathbb{C}_{0}$ is an object. For $N=\operatorname{Mon}(F)(M)$ and $y=F_{0}(x)$ we have a pullback square in Set, natural in $M$ and $x$ :

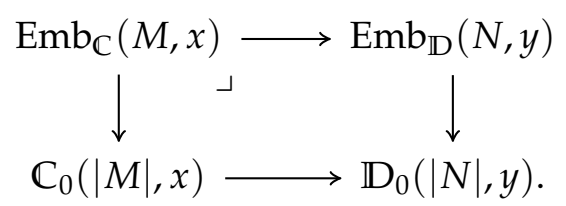

Definition 2.3.3. Let $M: c \rightarrow c$ be a monoid in an equipment $\mathbb{D}$. A collapse of $M$ is defined to be a universal embedding of $M$. That is, a collapse of $M$ is an object $\langle M\rangle \in \mathbb{D}_{0}$ together with an embedding

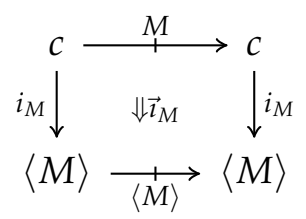

such that any other embedding of $M$ factors uniquely through $\vec{\imath}_{M}$ :

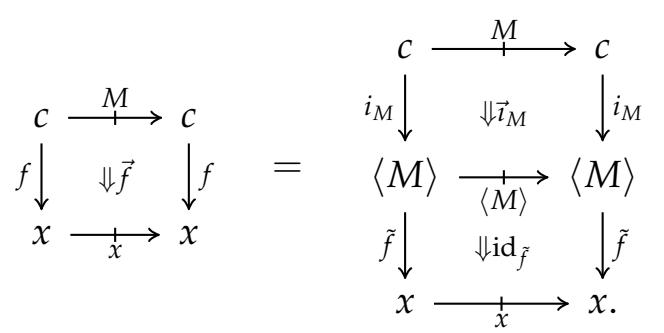

In other words, $\langle M\rangle$ represents the functor $\operatorname{Emb}(M,-): \mathbb{D}_{0} \rightarrow$ Set.

Example 2.3.4. Collapses of monoids always exist in Prof, and this is the prototypical example of collapse.

Consider a monoid $M: \mathscr{C} \rightarrow \mathscr{C}$ in Prof. The unit is a profunctor morphism $i: \operatorname{Hom}_{\mathscr{C}} \rightarrow M$. So for any $f: c \rightarrow d$ in $\mathscr{C}$ there is an element $i(f) \in M(c, d)$ such that

$$
g \cdot i(f) \cdot h=i(g \circ f \circ h)
$$

whenever this makes sense.

The multiplication $M \odot M \rightarrow M$ is an associative operation assigning to any elements $m_{1} \in M(c, d)$ and $m_{2} \in M(d, e)$ an element $m_{2} \bullet m_{1} \in M(c, e)$ satisfying the following equations whenever they make sense:

$$
\begin{gathered}
\left(f \cdot m_{2}\right) \bullet\left(m_{1} \cdot h\right)=f \cdot\left(m_{2} \bullet m_{1}\right) \cdot h \\
\left(m_{3} \cdot g\right) \bullet m_{1}=m_{3} \bullet\left(g \cdot m_{1}\right) \\
m \bullet i(f)=m \cdot f \quad \text { and } \quad i(g) \bullet m=g \cdot m
\end{gathered}
$$

Specifically, equations (18) and (19) simply say that • is a well-defined morphism $M \odot M \rightarrow M$, while (20) says that $\bullet$ is unital with respect to $i$. 
The collapse $\langle M\rangle$ of $M$ is the category with the same objects as $\mathscr{C}$, with morphisms $\langle M\rangle(c, d):=M(c, d)$, and with composition given by $\bullet$. The unit $i$ of $M$ gives a functor $i_{M}: \mathscr{C} \rightarrow\langle M\rangle$.

Remark 2.3.5. The equations (17)-(20) are actually overdetermined. It is easy to see that equations (18) and (19) follow from (20) and the associativity of $\bullet$. Thus, when proving that $\bullet: M \odot M \rightarrow M$ and $i:$ Hom $_{\mathscr{C}} \rightarrow M$ form a monoid, it suffices to prove (17), (20), and associativity of $\bullet$. These observations will be used to slightly simplify the proof of Proposition 3.4.9.

Remark 2.3.6. For any monoid $M: c \rightarrow c$, the companion $\widehat{i}_{M}: c \rightarrow\langle M\rangle$ (resp. the conjoint $\check{i}_{M}:\langle M\rangle \rightarrow c$ ) of the embedding $i_{M}: c \rightarrow\langle M\rangle$ has the structure of a left (resp. right) $M$-module. Indeed, the horizontal composition of $\vec{\imath}_{M}$ and the left hand cartesian 2-cell from (8) defining $\widehat{i}_{M}$ factors uniquely through some $l_{\hat{i}_{M}}$, as follows:

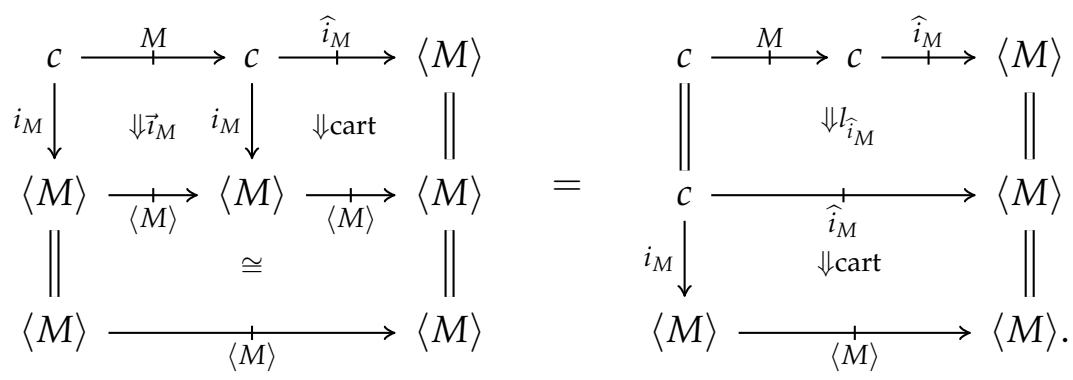

The right $M$-action on $\check{i}_{M}$ is obtained similarly.

Lemma 2.3.7. Let $M: c \rightarrow c$ and $N: d \rightarrow d$ be monoids in an equipment $\mathbb{D}$, and assume they admit collapses $\langle M\rangle$ and $\langle N\rangle$, respectively. Then restriction induces a functor

$$
\mathcal{H} \operatorname{Cor}(\mathbb{D})(\langle M\rangle,\langle N\rangle) \rightarrow{ }_{M} \operatorname{Bimod}_{N}
$$

Proof. For a proarrow $X:\langle M\rangle \rightarrow\langle N\rangle$ of $\mathbb{D}$, define $\tilde{X}: c \rightarrow d$ by the cartesian 2-cell

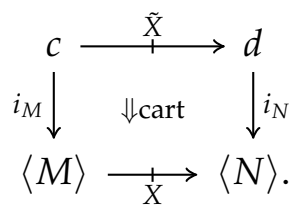

Then a 2-cell $X \Rightarrow Y$ immediately lifts to a 2-cell $\tilde{X} \Rightarrow \tilde{Y}$. Since (21) is cartesian, we obtain an equality
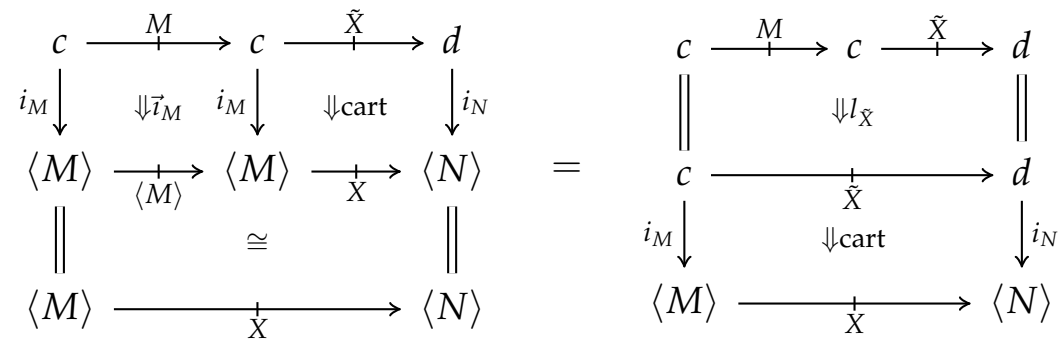

giving the action of $M$ on $\tilde{X}$. The action $r_{\tilde{X}}$ of $N$ on $\tilde{X}$ is obtained similarly, and one easily checks the axioms making $\tilde{X}$ an $(M, N)$-bimodule. 
Definition 2.3.8. [19, Proposition 5.4] An equipment $\mathbb{D}$ is exact if the following hold:

1. every monoid $M: c \rightarrow c$ has a collapse $\langle M\rangle$ with $\vec{\imath}_{M}$ cartesian;

2. for every pair of monoids $M$ and $N$ the restriction functor

$$
\mathcal{H} \operatorname{or}(\mathbb{D})(\langle M\rangle,\langle N\rangle) \stackrel{\simeq}{\longrightarrow}{ }_{M} \operatorname{Bimod}_{N}
$$

is an equivalence of categories.

Remark 2.3.9. The restriction functor (22) is clearly natural, giving a natural equivalence between pseudo-functors $\operatorname{Mon}(\mathbb{D})^{\mathrm{op}} \times \operatorname{Mon}(\mathbb{D})^{\mathrm{op}} \rightarrow$ Cat. Equivalently this gives an equivalence of fibrations, the inverse of which gives rise to a strictly-commuting pseudo-pullback square

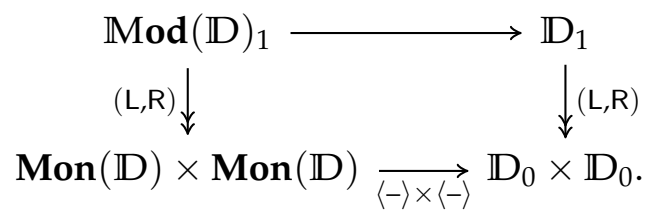

We will show in Proposition 2.3.12 that (under mild hypotheses) this preserves horizontal composition, thus defining a double functor and hence a local equivalence.

Example 2.3.10. It was proven in [19, Proposition 5.2] that for any equipment $\mathbb{D}$, its equipment $\operatorname{Mod}(\mathbb{D})$ of monoids and bimodules is exact. Thus Prof is exact, since there is an equivalence Prof $\cong \mathbb{M o d}($ Span $)$, where Span is the equipment of spans in Set; see [20].

Exact equipments arising in practice almost always have local reflexive coequalizers, and in this case it is possible to simplify the definition, as we show in Proposition 2.3.11. Recall from Remark 2.3.6 the natural $M$-module structures on the companion $\widehat{i}_{M}: c \rightarrow\langle M\rangle$ and conjoint $\breve{i}_{M}:\langle M\rangle \rightarrow c$ of the collapse embedding $i_{M}: c \rightarrow\langle M\rangle$. Recall also the notation $\mathrm{U}(a)$ from Definition 2.1.1, and $\eta, \epsilon$ from (9).

Proposition 2.3.11. Suppose $\mathbb{D}$ is an equipment with local reflexive coequalizers which satisfies Condition 1 of Definition 2.3.8. Then $\mathrm{D}$ satisfies Condition 2 if and only if for every monoid $M: c \rightarrow c$, the following diagram is a reflexive coequalizer in $\mathcal{H} \operatorname{Hor}(\mathbb{D})(\langle M\rangle,\langle M\rangle)$ :

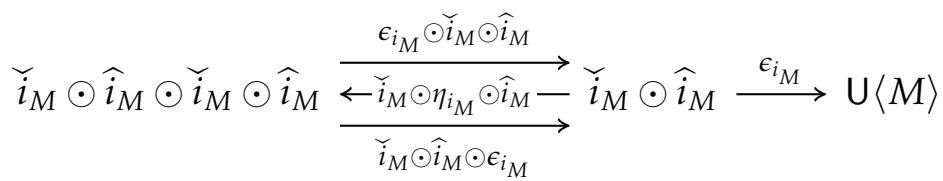

or, equivalently, $\check{i}_{M} \otimes_{M} \widehat{i}_{M} \cong \mathrm{U}\langle M\rangle$.

Proof. By Condition 1 of Definition 2.3.8, we have $M \cong \widehat{i}_{M} \odot \check{i}_{M}$, so the final equivalence is just the definition of horizontal composition in $\mathbb{M o d}(\mathbb{D})$; see Definition 2.2.4 and Remark 2.3.6. Below we will use the fact that $\otimes$ is defined as a reflexive coequalizer, and that, by definition of $\mathbb{D}$ having local reflexive coequalizers, $\odot$ preserves reflexive coequalizers in each variable. Finally, note that the restriction functor (22) is isomorphic 
to the functor $B \mapsto \widehat{i}_{M} \odot B \odot \breve{i}_{N}$, with the left and right actions given by the left action of $M$ on $\widehat{i}_{M}$ and right action of $N$ on $\breve{i}_{N}$.

Assuming $\breve{i}_{M} \otimes_{M} \widehat{i}_{M} \cong U\langle M\rangle$, we can construct an inverse to this restriction functor, sending an $(M, N)$-bimodule $B$ to $\check{i}_{M} \otimes_{M} B \otimes_{N} \widehat{i}_{N}$. It is easy to check that this gives an equivalence of categories:

$$
\begin{aligned}
\check{i}_{M} \otimes_{M}\left(\widehat{i}_{M} \odot B \odot \check{i}_{N}\right) \otimes_{N} \widehat{i}_{N} & \cong\left(\check{i}_{M} \otimes_{M} \widehat{i}_{M}\right) \odot B \odot\left(\check{i}_{N} \otimes_{N} \widehat{i}_{N}\right) \\
& \cong U\langle M\rangle \odot B \odot U\langle N\rangle \\
& \cong B
\end{aligned}
$$

and

$$
\begin{aligned}
\hat{i}_{M} \odot\left(\check{i}_{M} \otimes_{M} B \otimes_{N} \hat{i}_{N}\right) \odot \check{i}_{N} & \cong\left(\hat{i}_{M} \odot \check{i}_{M}\right) \otimes_{M} B \otimes_{N}\left(\widehat{i}_{N} \odot \check{i}_{N}\right) \\
& \cong M \otimes_{M} B \otimes_{N} N \\
& \cong B .
\end{aligned}
$$

Conversely, assuming the functor (22) is an equivalence of categories, then we can prove that $\breve{i}_{M} \otimes_{M} \widehat{i}_{M} \cong \mathrm{U}\langle M\rangle$ is an isomorphism by first applying the restriction functor:

$$
\begin{aligned}
\widehat{i}_{M} \odot\left(\check{i}_{M} \otimes_{M} \hat{i}_{M}\right) \odot \check{i}_{M} & \cong\left(\hat{i}_{M} \odot \check{i}_{M}\right) \otimes_{M}\left(\widehat{i}_{M} \odot \check{i}_{M}\right) \\
& \cong M \otimes_{M} M \\
& \cong M \\
& \cong \widehat{i}_{M} \odot \check{i}_{M} \\
& \cong \widehat{i}_{M} \odot U\langle M\rangle \odot \check{i}_{M} .
\end{aligned}
$$

Proposition 2.3.12. If $\mathbb{D}$ is an exact equipment with local reflexive coequalizers, then collapse induces an equipment functor $\langle-\rangle: \mathbb{M o d}(\mathbb{D}) \rightarrow \mathbb{D}$ which is a local equivalence.

Proof. It is easy to use the universal property of collapse to construct, from any monoid homomorphism $(f, \vec{f}):(c, M) \rightarrow(d, N)$, a vertical morphism $\langle f\rangle:\langle M\rangle \rightarrow\langle N\rangle$ in $\mathbb{D}$, thus defining a functor $\operatorname{Mon}(\mathbb{D}) \rightarrow \mathbb{D}_{0}$.

The functor $\langle-\rangle$ is defined on horizontal arrows and 2-cells as in Remark 2.3.9. It is straightforward to verify that this is a strong double functor, and hence a local equivalence, using the method of the proof of Proposition 2.3.11.

With these definitions in place we can now introduce two distinguished classes of vertical morphisms in an equipment $\mathbb{D}$. When $\mathbb{D}$ is exact, these will become the left and right classes in an orthogonal factorization system on $\mathcal{V e r t}(\mathbb{D})$.

Definition 2.3.13. [19, Definitions 4.3 and 4.5] Let $\mathbb{D}$ be an equipment and $f: c \rightarrow d$ a vertical morphism of $\mathbb{D}$. Consider the restriction square and unit square shown below:
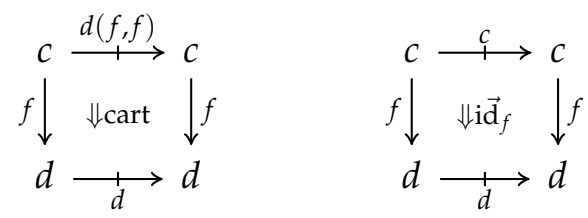
We say that $f$ is bo if the restriction square, where $d(f, f)$ has the induced monoid structure, is a collapse. We say that $f$ is ff if the unit square is cartesian.

In Section 3.2 we will define equipments of profunctors on monoidal categories, and we will verify their exactness directly in Section 3.3. The key ingredient in verifying that the equipment of traced profunctors is exact will be orthogonal factorization systems. Thus we briefly recall the notion of orthogonal factorization systems for 1-categories and strict 2-categories. Additional background on orthogonal factorization systems can be found in [3, Chapter 5.5]. The main result below is that exact equipments admit orthogonal factorization systems.

Definition 2.3.14. Let $\mathcal{V}$ be either Set or Cat, and suppose that $\mathscr{C}$ is a $\mathcal{V}$-enriched category. An orthogonal factorization system in $\mathscr{C}$ consists of two distinguished classes of morphisms, $(\mathscr{L}, \mathscr{R})$, with the following properties:

- Each morphism $f \in \mathscr{C}$ factors as $f=e \circ m$, where $m \in \mathscr{L}$ and $e \in \mathscr{R}$.

- If $m: a \rightarrow b$ in $\mathscr{L}$ and $e: c \rightarrow d$ in $\mathscr{R}$, then the left-hand square below is a pullback in $\mathcal{V}$ :
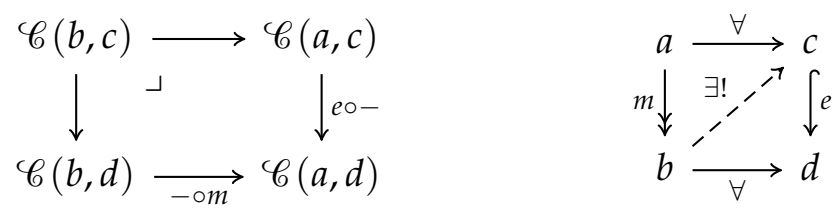

In particular, for all solid arrow squares, as in the right-hand diagram, there exists a unique diagonal filler. We say that $m$ is "left-orthogonal" to $e$, or that $e$ is "right-orthogonal" to $m$, and denote this relation as $m \nabla e$.

- If $m \nabla e$ for all $e \in \mathscr{R}$, then $m \in \mathscr{L}$. Likewise, if $m \nabla e$ for all $m \in \mathscr{L}$, then $e \in \mathscr{R}$.

As shown, we often indicate morphisms in $\mathscr{L}$ using a two-headed arrow and morphisms in $\mathscr{R}$ using a hooked arrow. ${ }^{2}$

Theorem 2.3.15. [19, Theorem 4.17] If an equipment $\mathbb{D}$ is exact, then the vertical 2-category $\operatorname{Vert}(\mathbb{D})$ admits a 2-orthogonal factorization system (bo,ff) as in Definition 2.3.13. In particular, there is an orthogonal factorization system (bo, ff) on the vertical 1-category $\mathbb{D}_{0}$.

In an exact equipment, there is a close connection between monoids and bo morphisms. This connection is formalized in Theorem 2.3.18 below, which is a key ingredient in the proofs of our main theorems.

Definition 2.3.16. Let $\mathbb{D}$ be an exact equipment. We define the equipment $\mathbb{D}^{\text {bo }}$ as follows: the vertical category $\mathbb{D}_{0}^{\text {bo }} \subseteq \mathbb{D}_{0}^{\rightarrow}$ is the full subcategory of the arrow category of $\mathbb{D}_{0}$ spanned by the arrows in the class bo. As such, we have functors dom, cod: $\mathbb{D}_{0}^{\text {bo }} \rightarrow$ $\mathbb{D}_{0}$. The rest of the structure of $\mathbb{D}^{\text {bo }}$ is defined by setting $\mathbb{D}^{\text {bo }}:=\operatorname{cod}^{*} \mathbb{D}$, i.e. by the

\footnotetext{
${ }^{2}$ We sometimes also use the two-headed arrow symbol $\rightarrow$ to indicate fibrations of categories (e.g. as we did in Theorem B or when defining the frame fibration for equipments, Definition 2.1.2). Whether we mean a bo map in an equipment or a fibration of categories should be clear from context.
} 
following strict pullback of categories (see Definition 2.1.5):

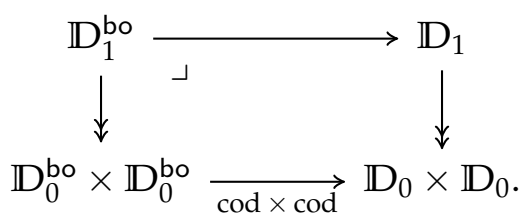

Section 3 begins with an outline of our proof of Theorem A as a sequence of equivalences. The last two results of the present section provide the step in that sequence which is not about traced or compact categories specifically, but in fact holds for any exact equipment. They form the bridge we need between profunctors and bijective-on-objects functors. They can be seen as a stronger statement of the idea (mentioned in the introduction to this section) that in an exact equipment $\mathbb{D}$, there is a very close connection between monoids in $\mathbb{D}$ and objects of $\mathbb{D}$.

Proposition 2.3.17. Let $\mathbb{D}$ be an exact equipment. There is an equivalence of fibrations on the left such that the triangle on the right also commutes:
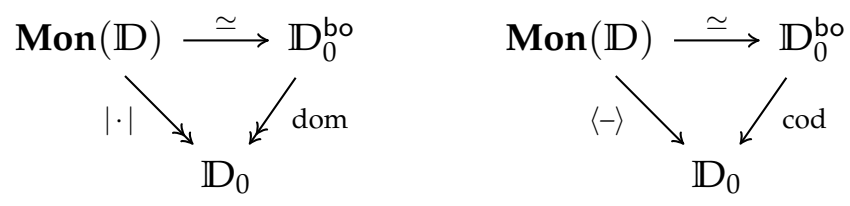

Proof. The functor dom: $\mathbb{D}_{0}^{\text {bo }} \rightarrow \mathbb{D}_{0}$ is a fibration via the factorization system in Theorem 2.3.15. The equivalence sends a monoid $(c, M)$ to the collapse morphism $i_{M}: c \rightarrow\langle M\rangle$, which is in bo by the exactness of $\mathbb{D}$. Since $\vec{\imath}_{M}$ is the universal embedding (16) of $M$, any monoid homomorphism $(f, \vec{f})$ gives rise to a unique $\tilde{f}$ such that

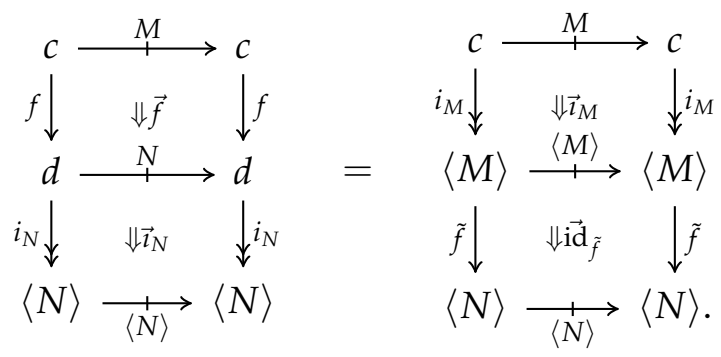

Moreover, the pair $(f, \tilde{f})$ defines a morphism of arrows $i_{M} \rightarrow i_{N}$ in $\mathbb{D}_{0}^{\text {bo }}$. By $[19$, Lemma $4.14]$, if $\vec{f}$ is cartesian then so is $\overrightarrow{i d}_{\tilde{f}}$, and clearly the converse also holds. It follows that the left triangle is a morphism of fibrations since $\vec{f}$ being cartesian over $f$ implies $(f, \tilde{f})$ is as well.

The inverse equivalence $\mathbb{D}_{0}^{\text {bo }} \rightarrow \operatorname{Mon}(\mathbb{D})$ sends a bo map $f: c \rightarrow d$ to the restriction $d(f, f)$ with its induced monoid structure.

Theorem 2.3.18. Let $\mathbb{D}$ be an exact equipment with local reflexive coequalizers. There is an equivalence of equipments

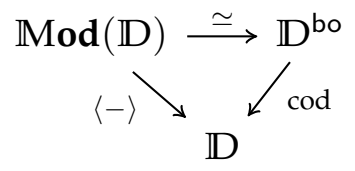


Proof. By Proposition 2.3.12 the equipment functor $\langle-\rangle: \mathbb{M o d}(\mathbb{D}) \rightarrow \mathbb{D}$ is a local equivalence, and cod: $\mathbb{D}^{\text {bo }} \rightarrow \mathbb{D}$ is a local equivalence by definition of $\mathbb{D}^{\text {bo }}$. It follows that the equivalence of fibrations from Proposition 2.3.17 extends to an equivalence of equipments.

\section{Equipments of monoidal profunctors}

With the needed material on general equipments out of the way, we now move on to topics specific to monoidal, traced monoidal, and compact closed categories, culminating in the proofs of Theorem A and Theorem B. In the introduction, we stated versions of these theorems for traced categories, but versions about compact categories seem to be their most natural and general statements. We will prove both.

After a brief review of monoidal, traced, and compact categories in Section 3.1, we define equipments $\mathbb{M n P r o f}$, TrProf, and $\mathbb{C}$ pProf in Section 3.2, whose objects are respectively monoidal, traced, and compact categories. Theorem B will be proven first through chains of equivalences of fibrations over CpCat and TrCat respectively as follows:

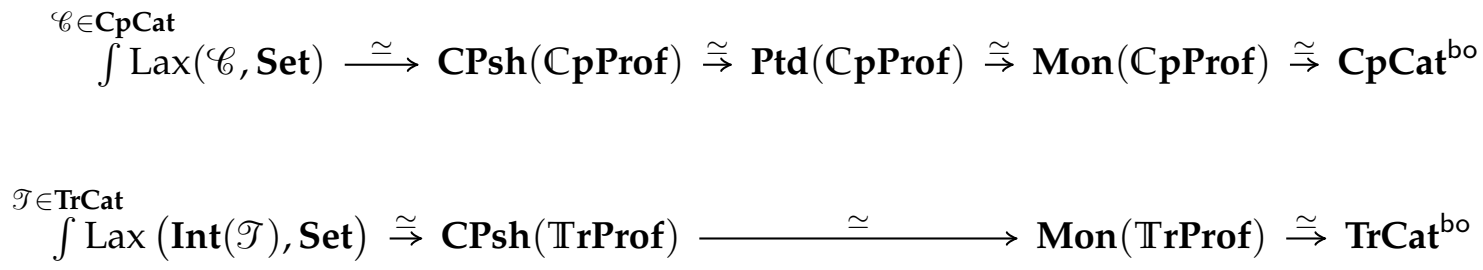

The leftmost equivalences follow trivially from the definition of CPsh, Definition 3.4.1. The rightmost equivalences will follow directly from Proposition 2.3.17 once we prove that TrProf and $\mathbb{C}$ pProf are exact in Section 3.3. The middle equivalences are specific to traced and compact categories; these are established in Section 3.4.

To prove Theorem A, we need to deal with the issue of objectwise-freeness. This is the purpose of Section 3.5. Finally in Section 3.6 we give our "traceless characterization of $\mathcal{T r C a t}^{\prime \prime}$.

\subsection{Monoidal, Compact, and Traced Categories}

We begin by reminding the reader of some categorical preliminaries: basic definitions and facts about monoidal, traced, and compact categories, lax and strong functors, and the Int construction. Standard references include [13], [11], and [12].

A strict monoidal category $\mathcal{M}$ is a category equipped with a functor $\otimes: \mathscr{M} \times \mathscr{M} \rightarrow \mathscr{M}$ and an object $I \in \mathscr{M}$, satisfying the usual monoid axioms. ${ }^{3}$ In other words, a strict monoidal category is a monoid object in the category Cat. Such a category $\mathcal{M}$ is symmetric if there are in addition natural isomorphisms

$$
\sigma_{X, Y}: X \otimes Y \rightarrow Y \otimes X
$$

\footnotetext{
${ }^{3}$ We also used the notation $\otimes$ to denote bimodule composition in Definition 2.2.4; hopefully the intended meaning of the symbol will be clear from context.
} 
satisfying equations $\sigma_{X, Y \otimes Z}=\left(\mathrm{id}_{X} \otimes \sigma_{X, Z}\right) \circ\left(\sigma_{X, Y} \otimes \mathrm{id}_{Z}\right)$ and $\sigma_{Y, X} \circ \sigma_{X, Y}=\mathrm{id}_{X \otimes Y}$.

Warning 3.1.1. Aside from the appendix, whenever we discuss monoidal categories in this article, we will mean symmetric strict monoidal categories.

Let $\mathscr{M}$ and $\mathcal{N}$ be monoidal categories. A functor $F: \mathscr{M} \rightarrow \mathcal{N}$ is called lax monoidal if it is equipped with coherence morphisms

$$
I_{\mathcal{N}} \stackrel{\mu}{\longrightarrow} F\left(I_{\mathcal{M}}\right) \quad \text { and } \quad F(X) \otimes_{\mathcal{N}} F(Y) \stackrel{\mu_{X, Y}}{\longrightarrow} F\left(X \otimes_{\mathcal{M}} Y\right)
$$

satisfying certain compatibility equations (see, e.g. [16, 4]). If all coherence morphisms are identities (resp. isomorphisms), then $F$ is strict (resp. strong). Let $\operatorname{Lax}(\mathscr{M}, \mathcal{N})$ denote the category of lax monoidal functors and monoidal transformations from $\mathcal{M}$ to $\mathcal{N}$.

Write $\mathcal{M n C a t}$ for the 2-category of strict symmetric monoidal categories, strict symmetric monoidal functors, and monoidal transformations. Let MnCat denote the underlying 1-category.

A compact category is a (symmetric) monoidal category $\mathscr{C}$ with the property that for every object $X \in \mathscr{C}$ there exists an object $X^{*}$ and morphisms $\eta_{X}: I \rightarrow X^{*} \otimes X$ and $\epsilon_{X}: X \otimes X^{*} \rightarrow I$ such that the following diagrams commute:
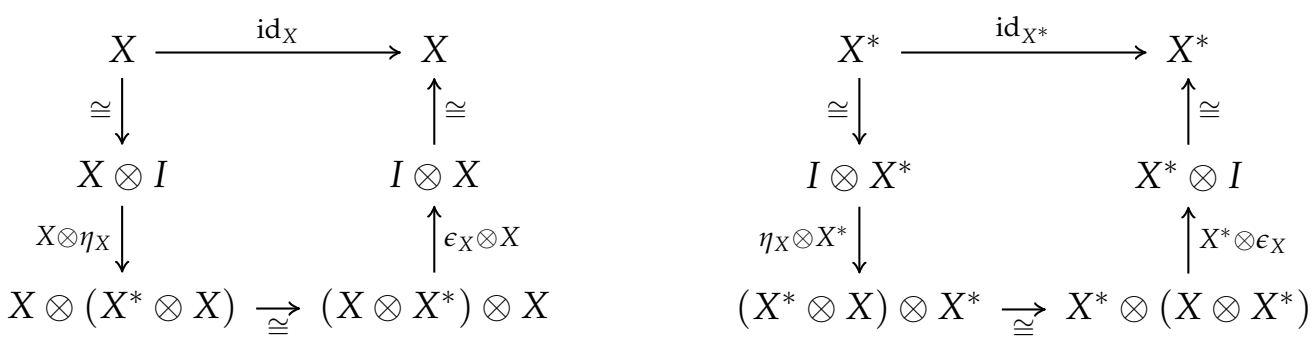

We will denote by $\mathcal{C}$ pat the full sub-2-category of $\mathcal{M}$ nCat spanned by the compact categories and write $\mathbf{U}_{\mathrm{CM}}$ : $\mathcal{E p C a t} \rightarrow \mathcal{M}$ nCat for the corresponding forgetful functor. Let $\mathrm{CpCat}$ denote the underlying 1-category.

Given a morphism $f: X \rightarrow Y$ in $\mathscr{C}$, we denote by $f^{*}: Y^{*} \rightarrow X^{*}$ the composite

$$
Y^{*} \stackrel{\eta_{X}}{\longrightarrow} X^{*} \otimes X \otimes Y^{*} \stackrel{f}{\rightarrow} X^{*} \otimes Y \otimes Y^{*} \stackrel{\epsilon_{Y}}{\rightarrow} X^{*} .
$$

It is easy to check that a strong functor $F: \mathscr{C} \rightarrow \mathscr{M}$ to a monoidal category preserves all duals that exist in $\mathscr{C}$, i.e. there is a natural isomorphism $F\left(c^{*}\right) \cong F(c)^{*}$. From this, it follows that if $F, G: \mathscr{C} \rightarrow \mathscr{C}^{\prime}$ are functors between compact categories, then any natural transformation $\alpha: F \rightarrow G$ is a natural isomorphism. Indeed, for any object $c \in \mathscr{b}$, the inverse of the $c$-component $\alpha_{c}: F c \rightarrow G c$ is given by the dual morphism $\left(\alpha_{c^{*}}\right)^{*}: G c \rightarrow F c$ to the dual component. Thus all 2-cells in $\mathbf{C p C a t}$ are invertible.

A trace structure on a (symmetric) monoidal category $\mathscr{T}$ is a collection of functions

$$
\operatorname{Tr}_{X, Y}^{U}: \operatorname{Hom}_{\mathscr{T}}(U \otimes X, U \otimes Y) \rightarrow \operatorname{Hom}_{\mathscr{T}}(X, Y)
$$

for $U, X, Y \in \mathrm{Ob}(\mathscr{T})$ satisfying seven equational axioms, we refer the reader to [12] for more details. If $\mathscr{T}$ and $\mathscr{U}$ are traced categories, then a (strict) traced functor is simply a strict symmetric monoidal functor which commutes with the trace operation. 
In [12], it is shown that every traced category $\mathscr{T}$ embeds as a full subcategory of a compact category $\operatorname{Int}(\mathscr{T})$ whose objects are pairs $\left(X^{-}, X^{+}\right) \in \mathrm{Ob}(\mathscr{T}) \times \mathrm{Ob}(\mathscr{T})$ with morphisms given by

$$
\operatorname{Hom}_{\operatorname{Int}(\mathscr{T})}\left(\left(X^{-}, X^{+}\right),\left(Y^{-}, Y^{+}\right)\right)=\operatorname{Hom}_{\mathscr{T}}\left(X^{-} \otimes Y^{+}, X^{+} \otimes Y^{-}\right)
$$

and with compositions computed using the trace of $\mathscr{T}$.

Remark 3.1.2. Traced categories were first defined in [12], which defines the 2-morphisms between traced functors to simply be monoidal transformations. However, this choice does not behave appropriately with the Int construction (for example Int would not be 2 -functorial). The error was corrected in [9], where it was shown that the appropriate 2-morphisms between traced functors are natural isomorphisms.

We denote by $\mathcal{T}$ rCat the corrected 2-category of traced categories (where 2-cells are invertible), and we denote its underlying 1-category by TrCat. Write $\mathbf{U}_{\mathrm{TM}}: \mathcal{T}$ rCat $\rightarrow$ $\mathcal{M n C a t}$ for the forgetful functor.

Every compact category $\mathscr{C}$ has a canonical trace structure, defined on a morphism $f: U \otimes X \rightarrow U \otimes Y$ morally (up to symmetries and identities) to be $\epsilon_{U} \circ f \circ \eta_{U}$. More precisely, one defines $\operatorname{Tr}_{X, Y}^{U}[f]$ to be the composite

$$
X \stackrel{\eta_{U} \otimes X}{\longrightarrow} U^{*} \otimes U \otimes X \stackrel{U^{*} \otimes f}{\longrightarrow} U^{*} \otimes U \otimes Y \stackrel{\sigma_{U^{*}, U} \otimes Y}{\longrightarrow} U \otimes U^{*} \otimes Y \stackrel{\epsilon_{U} \otimes Y}{\longrightarrow} Y
$$

Thus we have a functor $\mathbf{U}_{\mathrm{CT}}$ : $\mathcal{E p C a t} \rightarrow \mathcal{T}$ rCat. It is shown in [12] and [9] that this functor is the right half of a 2-adjunction

$$
\mathcal{T r C a t} \underset{\mathrm{U}_{\mathrm{CT}}}{\stackrel{\text { Int }}{\rightleftarrows}} \text { EpCat. }
$$

Note that $\mathbf{U}_{\mathrm{CM}}=\mathbf{U}_{\mathrm{CT}} \mathbf{U}_{\mathrm{TM}}$. In Section 3.6 we will be able to formally define the 2category $\mathcal{T}$ rCat without mentioning the trace structure (25) or the usual seven axioms, but instead in terms of the relationship between compact and monoidal categories.

Remark 3.1.3. We record the following facts, which hold for any traced category $\mathscr{T}$; each is shown in, or trivially derived from, [12]:

i. The component $\mathscr{T} \rightarrow \operatorname{Int}(\mathscr{T})$ of the unit of the adjunction (26) is fully faithful. It follows that Int: $\mathcal{T}$ rCat $\rightarrow \mathcal{C p C a t}$ is locally fully faithful.

ii. If $\mathscr{M}$ is a monoidal category and $F: \mathscr{M} \rightarrow \mathscr{T}$ is a fully faithful symmetric monoidal functor, then $\mathscr{M}$ has a unique trace for which $F$ is a traced functor.

iii. If $\mathscr{T}$ is compact then the counit $\operatorname{Int}(\mathscr{T}) \stackrel{\simeq}{\mathscr{T}}$ is an equivalence.

iv. Suppose that $\mathscr{T}^{\prime}$ is a traced category and that $F: \mathscr{T} \rightarrow \mathscr{T}^{\prime}$ is a traced functor. Then $F$ is bijective-on-objects (resp. fully faithful) if and only if $\operatorname{Int}(F)$ is.

\subsection{Monoidal profunctors}

Suppose $\mathscr{C}$ and $\mathscr{D}$ are monoidal categories. We define a monoidal profunctor $M$ from $\mathscr{C}$ to $\mathscr{D}$ to be an ordinary profunctor (see Example 2.1.6) $M: \mathscr{C}^{\mathrm{op}} \times \mathscr{D} \rightarrow$ Set which 
is equipped with a lax-monoidal structure, where Set is endowed with the cartesian monoidal structure. In the bimodule notation, this means that there is an associative operation assigning to any elements $m_{1} \in M\left(c_{1}, d_{1}\right)$ and $m_{2} \in M\left(c_{2}, d_{2}\right)$ an element $m_{1} \otimes m_{2} \in M\left(c_{1} \otimes c_{2}, d_{1} \otimes d_{2}\right)$ such that

$$
\left(f_{1} \cdot m_{1} \cdot g_{1}\right) \otimes\left(f_{2} \cdot m_{2} \cdot g_{2}\right)=\left(f_{1} \otimes f_{2}\right) \cdot\left(m_{1} \otimes m_{2}\right) \cdot\left(g_{1} \otimes g_{2}\right),
$$

as well as a distinguished element $I_{M} \in M(I, I)$ such that $I_{M} \otimes m=m=m \otimes I_{M}$ for any $m \in M(c, d)$. If moreover $m_{2} \otimes m_{1}=\sigma_{d_{1}, d_{2}} \cdot\left(m_{1} \otimes m_{2}\right) \cdot \sigma_{c_{1}, c_{2}}^{-1}$, then one says $M$ is symmetric monoidal. ${ }^{4}$

A monoidal profunctor morphism $\phi: M \rightarrow N$ is simply a monoidal transformation. Spelling this out in bimodule notation, $\phi$ is an ordinary morphism of profunctors such that $\phi\left(m_{1} \otimes m_{2}\right)=\phi\left(m_{1}\right) \otimes \phi\left(m_{2}\right)$ and $\phi\left(I_{M}\right)=I_{N}$.

We define a double category $\mathbb{M n P r o f}$ whose objects are (symmetric) monoidal categories, vertical arrows are strict (symmetric) monoidal functors, horizontal arrows are (symmetric) monoidal profunctors, and 2-cells are defined as in (12), requiring $\phi$ to be a monoidal transformation. It remains to check that the horizontal composition of monoidal profunctors is monoidal. This follows from the fact that reflexive coequalizers-namely the ones from (13) - commute with products in Set. Note that MnProf is in fact an equipment since the cartesian 2-cell (14) is a monoidal transformation if $N, F$, and $F^{\prime}$ are monoidal functors. We leave it as an exercise for the reader to check that there is an isomorphism of 2-categories $\mathcal{V e r t}(\mathbf{M n P r o f}) \cong \mathcal{M}$ nCat, i.e. that for any pair of strict symmetric monoidal functors $F, G: \mathscr{C} \rightarrow \mathscr{D}$, there is a bijection between monoidal transformations $\mathscr{C}(-,-) \rightarrow \mathscr{D}(F(-), G(-))$ and monoidal transformations $F \rightarrow G$.

The fully faithful functors $\mathrm{U}_{\mathrm{CM}}$ : CpCat $\rightarrow$ MnCat and Int: TrCat $\rightarrow$ CpCat, defined above, induce equipments $\mathbb{C}$ pProf $:=\mathbf{U}_{\mathrm{CM}}^{*}(\mathbb{M n P r o f})$ and TrProf $:=\mathbf{I n t}^{*}(\mathbb{C}$ pProf $)$ as in Definition 2.1.5. In particular, the vertical 1-categories of these equipments are given by

$$
\operatorname{MnProf}_{0}=\text { MnCat, } \operatorname{CpProf}_{0}=\text { CpCat }, \quad \operatorname{TrProf}_{0}=\text { TrCat. }
$$

It may seem strange at first to define a proarrow $\mathscr{T} \rightarrow \mathscr{T}^{\prime}$ between traced categories to be a monoidal profunctor $\operatorname{Int}(\mathscr{T}) \rightarrow \operatorname{Int}\left(\mathscr{T}^{\prime}\right)$. The next proposition serves as a first sanity check on this definition, and the remainder of this paper provides further support.

Proposition 3.2.1. There is an isomorphism of 2-categories, Vert $(\mathbb{T}$ rProf $) \cong \mathcal{T}$ rCat.

Proof. Clearly these 2-categories have the same underlying 1-category, so it suffices to show that there is a bijection $\mathcal{V e r t}(\operatorname{TrProf})(F, G) \cong \mathcal{T} \operatorname{rCat}(F, G)$ for any traced functors $F, G: \mathscr{T} \rightarrow \mathscr{T}^{\prime}$ which preserve units and composition. By the definition of TrProf, we have $\mathcal{V e r t}(\mathbb{T} \operatorname{rProf})(F, G)=\operatorname{CpCat}(\operatorname{Int}(F), \operatorname{Int}(G))$. The result then follows since Int is locally fully faithful [12].

Thus from the definitions and Proposition 3.2.1, we see that the vertical 2-categories of these equipments are as expected:

$$
\operatorname{Vert}(\mathbb{M n P r o f}) \cong \mathcal{M} \text { Cat }, \quad \mathcal{V e r t}(\mathbb{C} \text { Prof }) \cong \mathcal{C p C a t}, \quad \text { Vert }(\text { TrProf }) \cong \mathcal{T} \text { rCat }
$$

\footnotetext{
${ }^{4}$ We will generally suppress the word symmetric since all monoidal categories and monoidal profunctors are symmetric by assumption; see Warning 3.1.1.
} 
Proposition 3.2.2. Each of the equipments $\mathbb{M n P r o f}$, TrProf, and $\mathbb{C}$ Prof has local reflexive coequalizers.

Proof. It suffices to prove this for MnProf, since the other two are locally equivalent to it. For any monoidal category $\mathscr{M}$, the category of lax monoidal functors $\mathscr{M} \rightarrow$ Set is closed under reflexive coequalizers. This follows easily from the fact that reflexive coequalizers commute with finite products. (In fact, the same argument shows that the category $\mathbb{O}$-Alg of algebras for any colored operad $\mathbb{O}$ is closed under reflexive coequalizers.) Thus in particular the category of monoidal profunctors $\mathscr{M} \rightarrow \mathcal{N}$, i.e. the category of lax functors $\mathscr{M}^{\mathrm{op}} \times \mathcal{N} \rightarrow$ Set, is closed under reflexive coequalizers.

The fact that tensor product of monoidal profunctors preserves reflexive coequalizers follows from the fact that the tensor product itself is constructed as a reflexive coequalizer.

Remark 3.2.3. The equipments $\mathbb{M n P r o f}, \mathbb{T}$ rProf, and $\mathbb{C}$ Prof are in fact locally cocomplete. The category of profunctors $\mathscr{C} \rightarrow \mathscr{D}$ in any of these equipments is equivalent to the category of algebras for a monad on $\operatorname{Set}^{\mathscr{C}^{\text {op }} \times \mathscr{D}}$, and it is a general fact that if the category of algebras for a monad on a cocomplete category has reflexive coequalizers, then it has all colimits.

\subsection{MnProf, CpProf, and TrProf are exact}

Our next goal is to prove that the equipments MnProf, TrProf, and CpProf from Section 3.2 are all exact, as in Definition 2.3.8. We then discuss a few consequences of exactness: the (bo, ff) factorization systems (Proposition 3.3.6) and the relationship between monoids and bo-maps in these equipments (Corollary 3.3.7).

Proposition 3.3.1. The equipment $\mathbb{M n P r o f}$ is exact.

Proof. Suppose that $M: \mathscr{C} \rightarrow \mathscr{C}$ is a monoid in MnProf. One uses $M$ to construct a category $\langle M\rangle$ with the same objects as $\mathscr{C}$, and with hom sets defined by $\langle M\rangle(c, d):=$ $M(c, d)$ for any pair of objects $c, d \in \mathrm{Ob}(\mathscr{C})$. For any object $c$, the identity is provided by $i\left(\operatorname{id}_{\mathscr{C}}\right)$, while the multiplication $\bullet$ on $M$ defines composition in $\langle M\rangle$.

The unit of $M$ can also be used to construct an identity-on-objects functor $i_{M}: \mathscr{C} \rightarrow$ $\langle M\rangle$ and an embedding 2-cell $\vec{\imath}_{M}$ sending any element of $M$ to itself as a morphism of $\langle M\rangle$. It is easy to see that $\vec{\imath}_{M}$ is cartesian and that $\left(i_{M}, \vec{\imath}_{M}\right)$ is a collapse. The category $\langle M\rangle$ has a canonical monoidal structure, which on objects is just that of $\mathscr{C}$ and on morphisms is induced by the monoidal profunctor structure of $M$. It is also simple to verify the second part of Definition 2.3.8: an $(M, N)$-bimodule is precisely the data of a profunctor $\langle M\rangle \rightarrow\langle N\rangle$.

Proposition 3.3.2. The equipment $\mathbb{C}$ pProf is exact.

Proof. We can consider a monoid $M: \mathscr{C} \rightarrow \mathscr{C}$ in $\mathbb{C}$ pProf as a monoid in MnProf; it has a collapse embedding $i_{M}: M \rightarrow\langle M\rangle$ by Proposition 3.3.1. The collapse $\langle M\rangle$ is a monoidal category and, by Theorem $2.3 .18, i_{M}$ is a (strict symmetric monoidal) bo functor. But any strong monoidal functor preserves duals, so every object of $\langle M\rangle$ has a 
dual and hence $\langle M\rangle$ is compact. The map $\mathbf{U}_{\mathrm{CM}}: \mathbb{C}$ Prof $\rightarrow \mathbb{M n P r o f}$ is a fully faithful local equivalence and so $\langle M\rangle$ being a collapse in MnProf implies it is a collapse in CpProf.

We record the following consequence of Theorem 2.3.15 in the current notation.

Corollary 3.3.3. Each of the 2-categories $\mathcal{M n C a t}$ and $\mathcal{E p C a t}$ admits a 2-orthogonal (bo, ff) factorization system.

We abuse notation slightly and use the same name (bo,ff) for the factorization systems on different categories.

The exactness of TrProf is significantly more difficult to establish. We use the exactness of $\mathbb{C}$ pProf and the close relationship between TrProf and $\mathbb{C}$ pProf provided by the Int construction. We first prove a lemma which is needed for the proof. Recall the adjunction (26) and write $\eta_{\mathscr{T}}: \mathscr{T} \rightarrow \mathbf{U}_{\mathrm{CT}} \mathbf{I n t}(\mathscr{T})$ for the unit component on $\mathscr{T} \in \mathcal{T}$ rCat.

Lemma 3.3.4. Let $\mathscr{T}$ be a traced category, $\mathscr{C}$ a compact category, and $F: \operatorname{Int}(\mathscr{T}) \rightarrow \mathscr{C}$ a bijective-on-objects monoidal functor. Consider the factorization in MnCat of $\mathbf{U}_{\mathrm{CM}} F \circ \mathbf{U}_{\mathrm{TM}} \eta_{\mathcal{T}}$ into a bijective-on-objects $G$ followed by a fully faithful $H$, as follows:

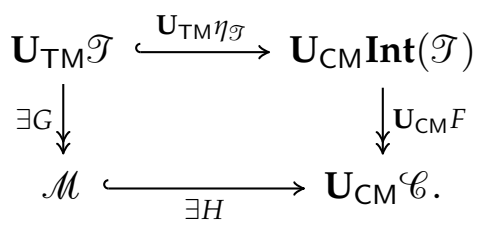

There is a unique trace structure on $\mathcal{M}$, i.e. a unique traced category $\mathscr{T}^{\prime}$ with $\mathbf{U}_{\mathrm{TM}} \mathscr{T}^{\prime}=\mathscr{M}$, such that the factorization lifts to TrCat:

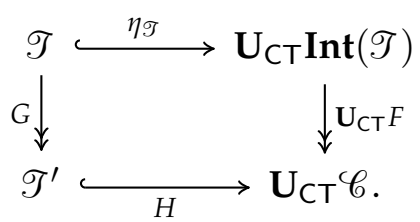

Moreover, there is an isomorphism $\alpha: \operatorname{Int}\left(\mathscr{T}^{\prime}\right) \cong \mathscr{C}$ such that $\mathbf{U}_{\mathrm{CT}} \alpha \circ \eta_{\mathscr{T}^{\prime}}=H$ and $\alpha \circ$ $\operatorname{Int}(G)=F$.

Proof. This derives mainly from basic properties of the Int construction; see Remark 3.1.3. Since $H: \mathscr{M} \rightarrow \mathbf{U}_{\mathrm{CM}} \mathscr{C}$ is fully faithful, the trace on $\mathbf{U}_{\mathrm{CT}} \mathscr{C}$ uniquely determines the desired trace structure on $\mathscr{T}^{\prime}$ by which $H$ is a traced functor. It also follows that $G$ respects the trace in $\mathscr{T}$ since $\mathbf{U}_{\mathrm{CT}} F \circ \eta_{\mathscr{T}}$ does.

For the final claim, consider the diagram

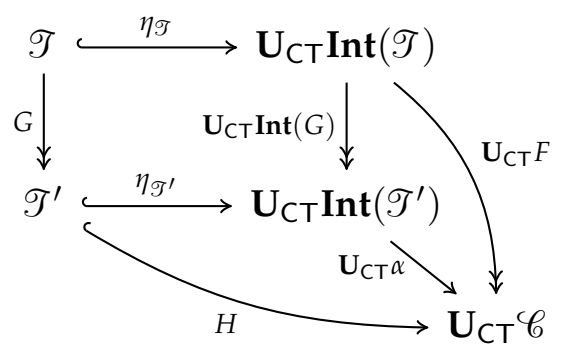


where $\alpha: \operatorname{Int}\left(\mathscr{T}^{\prime}\right) \rightarrow \mathscr{C}$ is the adjunct of $H$, which is fully faithful since $H$ is. Since $G$ is bo, $\mathbf{U}_{\mathrm{CT}} \operatorname{Int}(G)$ will be bo as well. But $\mathbf{U}_{\mathrm{CT}} F$ is bo, so $\mathbf{U}_{\mathrm{CT}} \alpha$ and hence $\alpha$ must be bo also. Since $\alpha$ is both $\mathrm{ff}$ and bo, it is an isomorphism, completing the proof.

Proposition 3.3.5. The equipment TrProf is exact.

Proof. Let $M: \mathscr{T} \rightarrow \mathscr{T}$ be a monoid in TrProf. By definition of $\mathbb{T r P r o f}$ this is a monoid $M: \operatorname{Int}(\mathscr{T}) \rightarrow \operatorname{Int}(\mathscr{T})$ in $\mathbb{C}$ Prof, so $M=\operatorname{Mon}(\operatorname{Int})(M)$ (in the language of Lemma 2.3.2). Define $\langle M\rangle_{C}$ and $\left(i_{M}, \vec{\imath}_{M}\right):(\operatorname{Int}(\mathscr{T}), M) \rightarrow\langle M\rangle_{C}$ to be the collapse embedding of $M$ in $\mathbb{C}$ pProf. Then applying Lemma 3.3.4 with $F=i_{M}$ gives a traced category $\langle M\rangle_{\mathrm{T}}$ and a bo functor $i_{M}^{\prime}: \mathscr{T} \rightarrow\langle M\rangle_{\mathrm{T}}$ as in the diagram below:

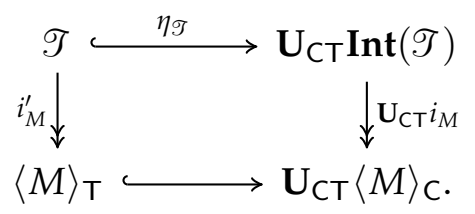

To see that $\langle M\rangle_{\mathrm{T}}$ is a collapse in TrProf we must establish the bijection $\operatorname{Emb}_{\operatorname{Tr}}\left(M, \mathscr{T}^{\prime}\right) \cong$ $\operatorname{TrCat}\left(\langle M\rangle_{\mathrm{T}}, \mathscr{T}^{\prime}\right)$, natural in the traced category $\mathscr{T}^{\prime}$.

Using the adjunction bijection and precomposition with the inverse of $\alpha: \operatorname{Int}\left(\langle M\rangle_{\mathrm{T}}\right) \cong$ $\langle M\rangle_{\mathrm{C}}$ from Lemma 3.3.4, we get an isomorphism

$$
\operatorname{TrCat}\left(\langle M\rangle_{\mathrm{T}}, \mathbf{U}_{\mathrm{C} T} \operatorname{Int}\left(\mathscr{T}^{\prime}\right)\right) \cong \mathbf{C p C a t}\left(\operatorname{Int}\left(\langle M\rangle_{\mathrm{T}}\right), \operatorname{Int}\left(\mathscr{T}^{\prime}\right)\right) \cong \mathbf{C p C a t}\left(\langle M\rangle_{\mathrm{C}}, \operatorname{Int}\left(\mathscr{T}^{\prime}\right)\right)
$$

This isomorphism is the top right morphism in the diagram

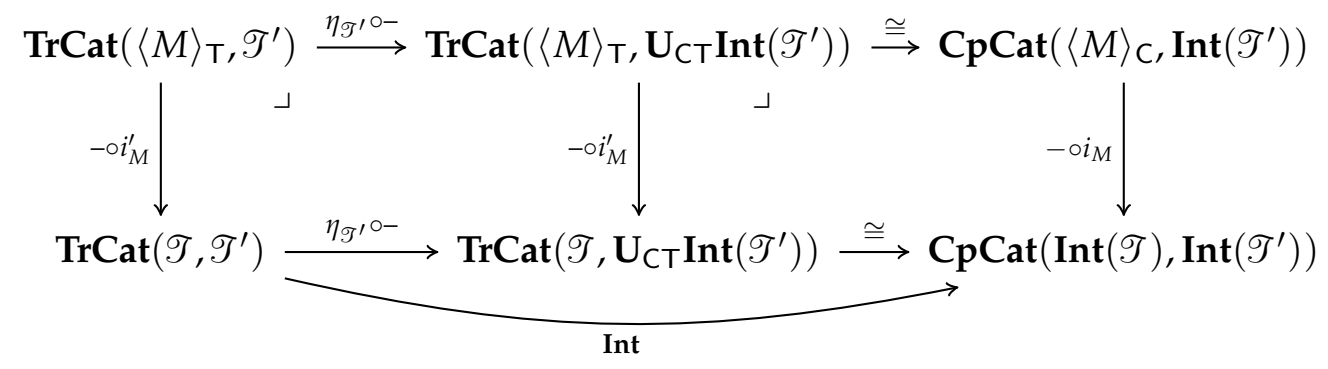

The right square commutes by the naturality of the (Int, $\mathbf{U}_{\mathrm{CT}}$ ) adjunction, together with the equality $i_{M}=\alpha \circ \operatorname{Int}\left(i_{M}^{\prime}\right)$ from Lemma 3.3.4. The left square is a pullback, by the orthogonality of $i_{M}^{\prime} \in$ bo and $\eta_{\mathscr{T}^{\prime}} \in \mathrm{ff}$, and the right square is a pullback because the top and bottom maps are isomorphisms. Hence the outer square is a pullback as well. Since $\langle M\rangle_{\mathrm{C}}$ is a collapse in $\mathbb{C}$ pProf, there is a bijection $\operatorname{Emb}_{\mathrm{Cp}}\left(M, \operatorname{Int}\left(\mathscr{T}^{\prime}\right)\right) \cong$ CpCat $\left(\langle M\rangle_{\mathrm{C}}, \operatorname{Int}\left(\mathscr{T}^{\prime}\right)\right)$ so by Lemma 2.3.2, the outer pullback produces the desired natural isomorphism $\operatorname{Emb}_{\operatorname{Tr}}\left(M, \mathscr{T}^{\prime}\right) \cong \operatorname{TrCat}\left(\langle M\rangle_{\mathrm{T}}, \mathscr{T}^{\prime}\right)$.

Since the trivial monoid on $\langle M\rangle_{\mathrm{T}}$ in TrProf is by definition the trivial monoid on $\operatorname{Int}\left(\langle M\rangle_{\mathrm{T}}\right) \cong\langle M\rangle_{\mathrm{C}}$ in $\mathbb{C}$ pProf, the collapse embedding $M \Rightarrow\langle M\rangle_{\mathrm{T}}$ is (after composition with the isomorphism $\alpha$ ) just the collapse $\left(i_{M}, \vec{\imath}_{M}\right)$ in CpProf, and hence is cartesian. Since the inclusion TrProf $\rightarrow \mathbb{C}$ pProf is a local equivalence and CpProf is exact, the second condition of Definition 2.3.8 follows immediately. 
Proposition 3.3.6. In $\mathbb{M n P r o f}$, $\mathbb{T r P r o f}$, and $\mathbb{C}$ Prof, a vertical map is $\mathrm{ff}$ (resp. bo) if and only if it is fully faithful (resp. bijective-on-objects) in the usual sense.

Proof. It is clear that the forgetful double functor $U: \mathbb{M n P r o f} \rightarrow \mathbb{P r o f}$ creates cartesian 2-cells: a 2-cell in $\mathbb{M n P r o f}$ is cartesian if and only if its underlying 2-cell in $\mathbb{P r o f}$ is cartesian. In particular, this implies that a vertical map in $\mathbb{M n P r o f}$ is ff if and only if its underlying map in Prof is ff, hence is fully faithful in the usual sense.

By the construction of collapses in $\mathbb{M n P r o f}$, it is easy to see that $U$ similarly creates collapse 2-cells. Thus a vertical map in $\mathbb{M n P r o f}$ is bo if and only if its underlying map in Prof is bo, hence is bijective-on-objects in the usual sense.

Because the forgetful double functor $\mathbf{U}_{\mathrm{CM}}$ : $\mathrm{CpProf} \rightarrow \mathbb{M n P r o f}$ is a fully faithful local equivalence, it follows that it too creates cartesian 2-cells, and from the construction of collapses in CpProf it also creates collapse 2-cells. Hence a vertical map in $\mathbb{C p P r o f}$ is in bo/ff if and only if its underlying map in $\mathbb{M n P r o f}$ is.

Likewise, Int: $\mathbb{T}$ rProf $\rightarrow \mathbb{C}$ PProf creates cartesian and collapse 2-cells. It only remains to show that a traced functor $F: \mathscr{T} \rightarrow \mathscr{T}^{\prime}$ is fully faithful (resp. bijective-onobjects) in the usual sense if and only if $\operatorname{Int}(F)$ is. For fully faithfulness, this follows easily from the fact that the unit $\eta: \mathscr{T} \rightarrow \mathbf{U}_{\mathrm{CT}} \mathbf{I n t} T$ is fully faithful. It is also clear that $\operatorname{Int}(F)$ is bijective-on-objects by construction when $F$ is. Finally, suppose $\operatorname{Int}(F)$ is bijective-on-objects. Because the unit $\eta$ is injective-on-objects, $F$ must be injectiveon-objects. If $x \in \mathscr{T}^{\prime}$ is any object, then there is an object $\left(t_{1}, t_{2}\right) \in \operatorname{Int} T$ such that $\operatorname{Int}(F)\left(t_{1}, t_{2}\right)=(x, I)$, but $\operatorname{Int}(F)\left(t_{1}, t_{2}\right)=\left(F t_{1}, F t_{2}\right)$, hence $F t_{1}=x$, showing that $F$ is also surjective-on-objects.

Finally, we record for reference the application of Theorem 2.3.18 to the exact equipments TrProf and CpProf.

Corollary 3.3.7. There are equivalences of equipments

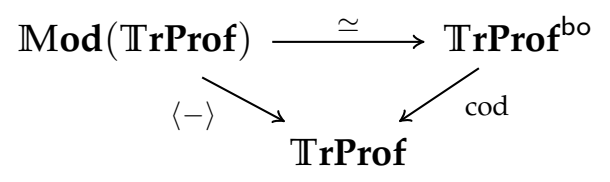

and

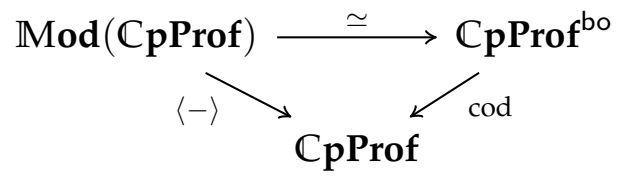

\subsection{Special properties of $\mathbb{C}$ Prof}

In Section 3.3, we saw that the equipments TrProf and $\mathbb{C}$ pProf are exact, connecting traced and compact categories to the machinery reviewed in Section 2. All that has come before can be seen as general machinery used to translate the statement of Theorem B into a more convenient form. In this section, we collect the properties unique to traced and compact categories, and use these to prove Theorem B via this translation.

Before we get to that, though, we must quickly say how we talk about (co)presheaves in the language of equipments.

\subsubsection{Internal copresheaves and endo-proarrows in an equipment}

Copresheaves on a category $\mathscr{C}$ can be identified with profunctors $1 \rightarrow \mathscr{C}$ in Prof. Motivated by this, we will think of proarrows $1 \rightarrow c$ in any equipment $\mathbb{D}$ with a 
terminal object 1 as "internal copresheaves" on the object $c$. For each object, there is a category of copresheaves $\mathcal{H} \operatorname{or}(\mathbb{D})(1, c)$. We can give a direct construction of the bifibration over $\mathbb{D}_{0}$ whose fiber over an object $c$ is the category of copresheaves on $c$ :

Definition 3.4.1. Let $\mathbb{D}$ be an equipment with a terminal object $1 \in \mathbb{D}_{0} .{ }^{5}$ We define the category $\operatorname{CPsh}(\mathbb{D})$, bifibered over $\mathbb{D}_{0}$, by the strict pullback of categories

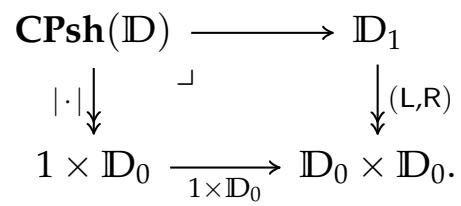

Lemma 3.4.2. Let $F: \mathbb{C} \rightarrow \mathbb{D}$ be an equipment functor. Suppose that $\mathbb{C}_{0}$ and $\mathbb{D}_{0}$ have terminal objects which are preserved by $F_{0}$. Then there is an induced morphism of fibrations

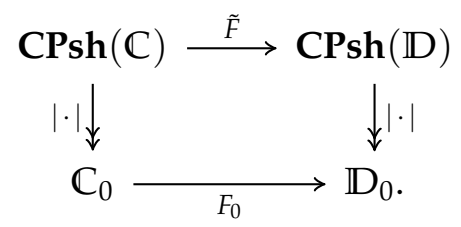

Moreover, if $F$ is a local equivalence, then (28) is a pseudo-pullback.

Proof. Consider the cube

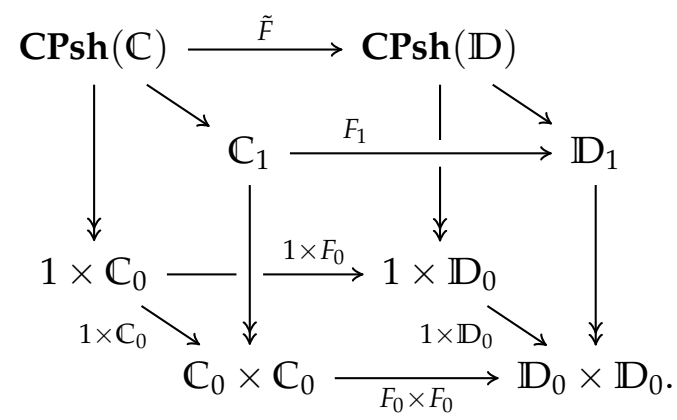

Since $F_{0}$ preserves terminal objects, the bottom face of the cube commutes. The left and right faces of the cube are strict pullbacks by definition, hence there is a unique $\tilde{F}$ making the cube commute.

If $F$ is a local equivalence, then the front face is a pseudo-pullback. The left and right faces are strict pullbacks along fibrations, hence pseudo-pullbacks (see Remark 2.1.4). It follows that the back face is a pseudo-pullback as well.

Definition 3.4.3. Given an equipment $\mathbb{D}$, we define the fibration of endo-proarrows by the strict pullback

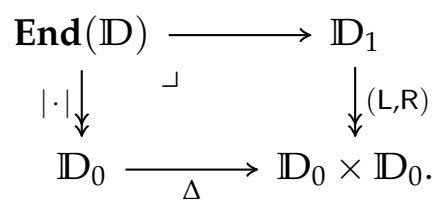

\footnotetext{
${ }^{5}$ In fact, such a definition makes sense for any object of $d \in \mathbb{D}_{0}$, but we will only use the case $d=1$.
} 
We also define a fibration $\operatorname{Ptd}(\mathbb{D}) \rightarrow \mathbb{D}_{0}$ whose objects are pointed endo-proarrows, i.e. endo-proarrows $M: c \rightarrow c$ in $\mathbb{D}$ equipped with a unit $i_{M}: U(c) \Rightarrow M$ as in (15) (but not a multiplication), and whose morphisms are 2-cells which preserve the units.

\subsubsection{Copresheaves coincide with monoids in $\mathbb{C}$ pProf and TrProf}

The component of the proof of Theorem B which is specific to traced and compact categories is the following equivalence of fibrations:

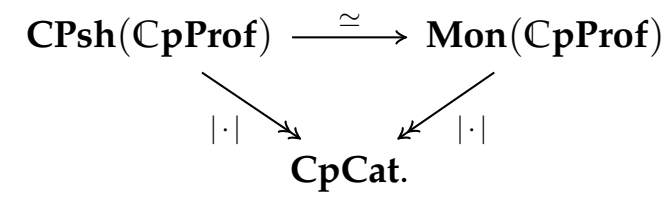

We prove this equivalence in this section (which implies the corresponding equivalence for traced categories). To do so we introduce a third fibration-that of pointed endoproarrows - and establish its equivalence with each of $\mathbf{C P s h}(\mathbb{C}$ pProf $)$ and $\mathbf{M o n}(\mathbb{C}$ pProf $)$ in Proposition 3.4.8 and Proposition 3.4.9 below. The introduction of $\operatorname{Ptd}(\mathbb{C}$ pProf $)$ is merely a convenient way of organizing the proof: recovering a copresheaf from a monoidal endo-profunctor requires only a unit (and not a multiplication), while for any monoidal endo-profunctor on a compact category, a unit extends uniquely to a multiplication.

Remark 3.4.4. One can think of compact categories as a categorification of groups, where duals of objects act like inverses of group elements. From this perspective, the results of this section can be seen as categorifications of basic facts from group theory.

We can think of profunctors between compact categories as playing the role of relations between groups which are stable under multiplication. Pointed endo-profunctors act like reflexive relations, and monoids in profunctors act like reflexive and transitive relations. In fact, one can define an equipment of groups, group homomorphisms, and equivariant relations, in which monoids are precisely reflexive transitive relations. It is easy to see that copresheaves, i.e. equivariant relations $1 \rightarrow G$, are the same as subgroups of $G$.

In this way, the equivalence $\operatorname{CPsh}(\mathbb{C}$ Prof $) \simeq \operatorname{Mon}(\mathbb{C}$ pProf $)$ categorifies the standard fact that a subgroup determines, and is determined by, the conjugacy congruence. The equivalence $\operatorname{Ptd}(\mathbb{C}$ pProf $) \simeq \operatorname{Mon}(\mathbb{C}$ prof $)$ would seem to be saying that every reflexive relation (stable under multiplication) on a group is in fact transitive, which while true is perhaps less familiar than the conjugacy relation.

Note that in the definition of a Mal'cev category (see [5]) the last property above is singled out as characterizing categories in which some amount of classical group theory can be developed. By analogy, we might think of this section as proving that $\mathbb{C}$ pProf is a "Mal'cev equipment".

It will be helpful to work out what a monoid in MnProf looks like using the bimodule notation for profunctors. A unit for a monoidal profunctor $M: \mathscr{C} \rightarrow \mathscr{C}$ is a unit $i: \operatorname{Hom}_{\mathscr{C}} \rightarrow M$ as in Example 2.3.4 where

$$
i\left(\mathrm{id}_{I_{\mathscr{C}}}\right)=I_{M} \quad \text { and } \quad i(f \otimes g)=i(f) \otimes i(g)
$$


for any morphisms $f$ and $g$ in $\mathscr{C}$. Similarly, the multiplication $\bullet$ on $M$ must satisfy

$$
\begin{gathered}
I_{M} \bullet I_{M}=I_{M} \\
\left(m_{2} \otimes m_{2}^{\prime}\right) \bullet\left(m_{1} \otimes m_{1}^{\prime}\right)=\left(m_{2} \bullet m_{1}\right) \otimes\left(m_{2}^{\prime} \bullet m_{1}^{\prime}\right)
\end{gathered}
$$

for any $m_{1} \in M(c, d), m_{1}^{\prime} \in M\left(c^{\prime}, d^{\prime}\right), m_{2} \in M(d, e)$, and $m_{2}^{\prime} \in M\left(d^{\prime} e^{\prime}\right)$, in addition to the requirements from Example 2.3.4.

Remark 3.4.5. Equation (30) follows immediately from (20) and the identification $i\left(\operatorname{id}_{I_{\mathscr{G}}}\right)=I_{M}$. Thus, to prove that $i$ and $\bullet$ form a monoid in MnProf, it suffices to show (29) and (31), in addition to the requirements discussed in Remark 2.3.5.

The next lemma shows that for any compact category $\mathscr{C}$ the standard natural equivalence $\operatorname{Hom}_{\mathscr{C}}(a, b) \simeq \operatorname{Hom}_{\mathscr{C}}\left(I, a^{*} \otimes b\right)$ can be extended to any pointed monoidal endo-profunctor on $\mathscr{C}$.

Lemma 3.4.6. Let $\mathscr{C}$ be a compact category. Given any pointed endo-profunctor $i$ : $\operatorname{Hom}_{\mathscr{C}} \rightarrow$ $M$ in $\operatorname{Ptd}(C \mathbf{C P r o f})$, there is a natural bijection $M(a, b) \cong M\left(I, a^{*} \otimes b\right)$ for any objects $a, b \in \mathscr{C}$.

Proof. Given $m \in M(a, b)$, we can construct an element

$$
\left(i\left(\operatorname{id}_{a^{*}}\right) \otimes m\right) \cdot \eta_{a} \in M\left(I, a^{*} \otimes b\right) .
$$

Conversely, given $m^{\prime} \in M\left(I, a^{*} \otimes b\right)$, we can construct an element

$$
\left(\epsilon_{a} \otimes \mathrm{id}_{b}\right) \cdot\left(i\left(\mathrm{id}_{a}\right) \otimes m^{\prime}\right) \in M(a, b) .
$$

It is simple to check that this defines a natural bijection.

With the fibration $|\cdot|:$ End (CpProf) $\rightarrow$ CpCat from Definition 3.4.3, we can define the functors

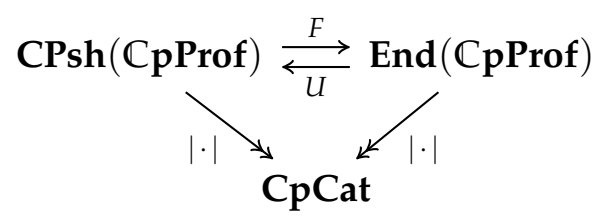

where $F M: \mathscr{C}^{\text {op }} \times \mathscr{C} \rightarrow$ Set is defined by $F M(a, b):=M\left(a^{*} \otimes b\right)$ while $U N: \mathscr{C} \rightarrow$ Set is given by $U N(a):=N(I, a)$. It is simple to check that $F$ and $U$ are morphisms of fibrations, i.e. that they preserve cartesian morphisms.

Proposition 3.4.7. The functor F: CPsh(CpProf $) \rightarrow \operatorname{End}(\mathbb{C} p P r o f)$ factors through $\operatorname{Ptd}(\mathbb{C}$ Prof $)$.

Proof. Let $M: \mathscr{C} \rightarrow$ Set be an object in CPsh (CpProf). Since $M$ is a monoidal profunctor, there is a given unit element $I_{M} \in M(I)$. Thus given any $f: c \rightarrow d$ in $\mathscr{C}$, we can define the element $i(f) \in F M(c, d)=M\left(c^{*} \otimes d\right)$ via

$$
i(f):=\left(\left(\operatorname{id}_{c^{*}} \otimes f\right) \circ \eta_{c}\right) \cdot I_{M} .
$$

It is easy to check that this construction of a unit $i$ is functorial. 
Thus, we have induced functors $F, U: \mathrm{CPsh}(\mathrm{C}$ Prof $) \leftrightarrows \operatorname{Ptd}(\mathbb{C}$ Prof $)$ giving the diagram

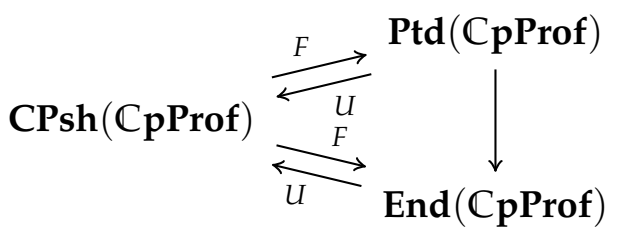

in which the triangle involving the $F^{\prime}$ s and the triangle involving the $U^{\prime}$ s both commute.

Proposition 3.4.8. The functors $F$ and $U$ from (33) form an equivalence of fibrations

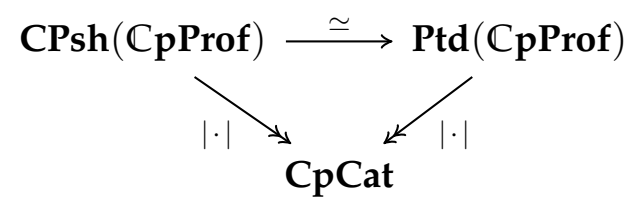

Proof. If $M \in \mathbf{C P s h}(\mathbb{C}$ pProf), i.e. $M$ is a lax functor $\mathscr{C} \rightarrow$ Set for some compact $\mathscr{C}$, then $U(F M)(a)=(F M)(I, a)=M\left(I^{*} \otimes a\right) \cong M(a)$ for any $a \in \mathscr{C}$. On the other hand, given $N \in \operatorname{Ptd}\left(\right.$ CpProf), we have $F(U N)(a, b)=N\left(I, a^{*} \otimes b\right)$, and the equivalence follows from Lemma 3.4.6.

To make the proof of Proposition 3.4.9 easier to follow, we make use of an extension of the standard string diagrams for (compact) monoidal categories to monoidal profunctors, as well as monoids in MnProf. We summarize the use of these string diagrams in Table 1. We will only use these diagrams in the proof of Proposition 3.4.9, and there only informally, as an aid to follow the rigorous equational proofs.

Proposition 3.4.9. The forgetful functor $\operatorname{Mon}(\mathbb{C}$ Prof $) \rightarrow \operatorname{Ptd}(C p P r o f)$ is an equivalence of fibrations over CpCat.

Proof. It is clear that this forgetful functor, which we refer to as $U$ in the proof, is a morphism of fibrations, so we must show that $U$ is an equivalence of categories.

To define an inverse functor $U^{-1}$, consider an object of $\operatorname{Ptd}(\mathbf{C p P r o f})$, i.e. a profunctor $N: \mathscr{C} \rightarrow \mathscr{C}$ with basepoint $i: \operatorname{Hom}_{\mathscr{C}} \rightarrow N$. We can define a multiplication on $N$ by the formula

$$
n_{2} \bullet n_{1}:=\left(\epsilon_{d} \otimes \mathrm{id}_{e}\right) \cdot\left(n_{1} \otimes i\left(\mathrm{id}_{d^{*}}\right) \otimes n_{2}\right) \cdot\left(\mathrm{id}_{c} \otimes \eta_{d}\right)
$$

for any $n_{1} \in N(c, d)$ and $n_{2} \in N(d, e)$, or in picture form:

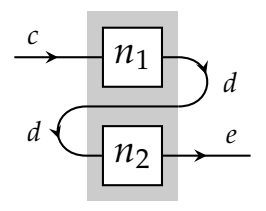

It is straightforward to check that this multiplication is associative. Remark 3.4.5 says that, in order to show that $N$ together with $i$ and $\bullet$ define an object in Mon(CpProf), 


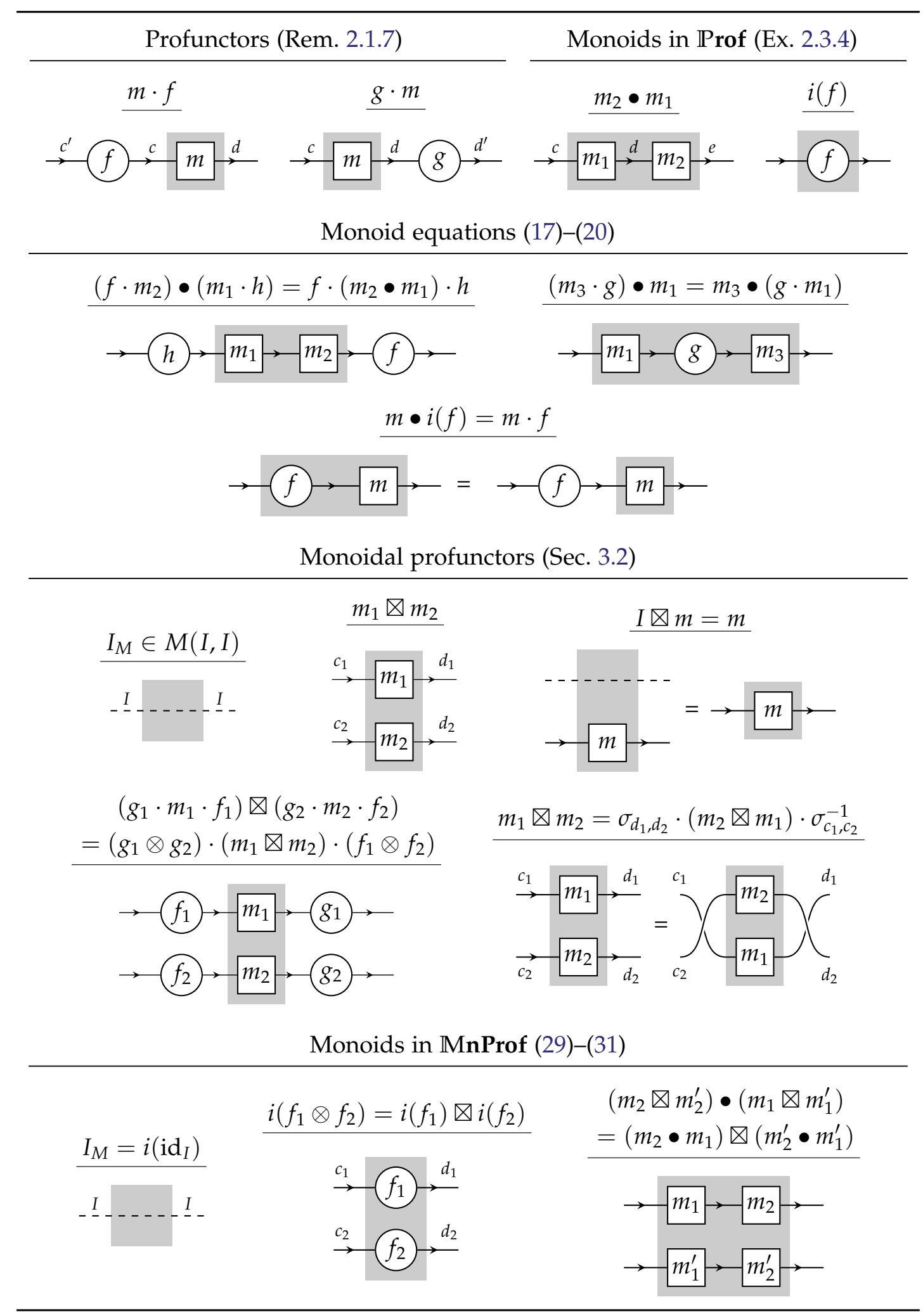

Table 1: String diagrams for structured profunctors. 
we must additionally show that this multiplication satisfies the equations (20) and (31). We will begin by showing that $n \bullet i(f)=n \cdot f$ for any $n \in N(d, e)$ and $f: c \rightarrow d$ :

$$
\begin{aligned}
& \text { re } \\
& \begin{aligned}
n \bullet i(f) & =\left(\epsilon_{d} \otimes \mathrm{id}_{e}\right) \cdot\left(i(f) \otimes i\left(\mathrm{id}_{d^{*}}\right) \otimes n\right) \cdot\left(\mathrm{id}_{c} \otimes \eta_{d}\right) \\
& =\left(\epsilon_{d} \otimes \mathrm{id}_{e}\right) \cdot\left(i\left(f \otimes \mathrm{id}_{d^{*}}\right) \otimes n\right) \cdot\left(\mathrm{id}_{c} \otimes \eta_{d}\right) \\
& =\left(\left(\epsilon_{d} \cdot i\left(f \otimes \mathrm{id}_{d^{*}}\right)\right) \otimes\left(\mathrm{id}_{e} \cdot n\right)\right) \cdot\left(\mathrm{id}_{c} \otimes \eta_{d}\right) \\
& =\left(i\left(\epsilon_{d} \circ\left(f \otimes \mathrm{id}_{d^{*}}\right)\right) \otimes\left(n \cdot \mathrm{id}_{d}\right)\right) \cdot\left(\mathrm{id}_{c} \otimes \eta_{d}\right) \\
& =\left(i\left(\mathrm{id}_{I}\right) \otimes n\right) \cdot\left(\left(\left(\epsilon_{d} \circ\left(f \otimes \mathrm{id}_{d^{*}}\right)\right) \otimes \mathrm{id}_{d}\right) \circ\left(\mathrm{id}_{c} \otimes \eta_{d}\right)\right) \\
& =\left(I_{N} \otimes n\right) \cdot\left(\left(\epsilon_{d} \otimes \mathrm{id}_{d}\right) \circ\left(\mathrm{id}_{d} \otimes \eta_{d}\right) \circ\left(f \otimes \mathrm{id}_{I}\right)\right) \\
& =\left(I_{N} \otimes n\right) \cdot\left(f \otimes \mathrm{id}_{I}\right) \\
& =n \cdot f .
\end{aligned}
\end{aligned}
$$

The equation $i(f) \bullet n=f \cdot n$ follows similarly, so we have verified (20).

Finally, we must check (31). Recall that this says

$$
\left(n_{2} \otimes n_{2}^{\prime}\right) \bullet\left(n_{1} \otimes n_{1}^{\prime}\right)=\left(n_{2} \bullet n_{1}\right) \otimes\left(n_{2}^{\prime} \bullet n_{1}^{\prime}\right)
$$

for any $n_{1} \in N(c, d), n_{1}^{\prime} \in N\left(c^{\prime}, d^{\prime}\right), n_{2} \in N(d, e)$, and $n_{2}^{\prime} \in N\left(d^{\prime}, e^{\prime}\right)$, which we prove below:

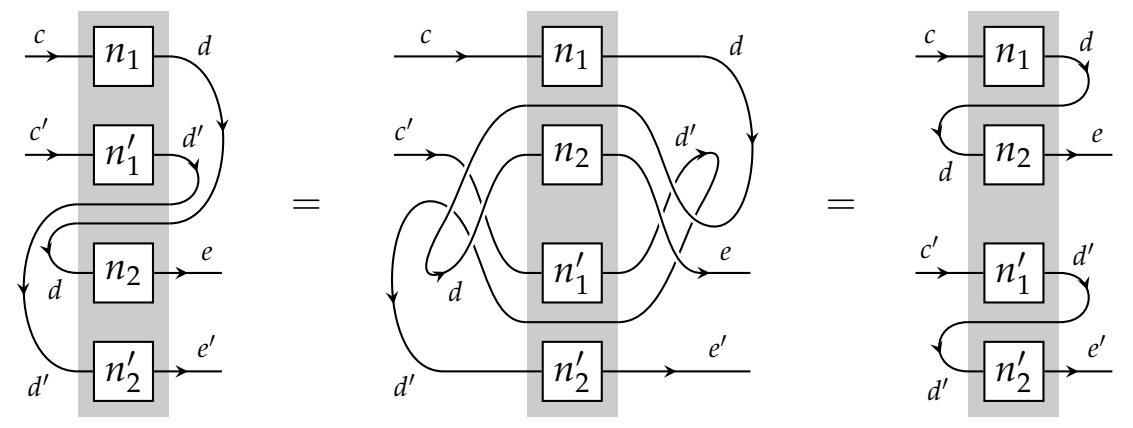

$$
\begin{aligned}
&\left(n_{2} \otimes\right.\left.n_{2}^{\prime}\right) \bullet\left(n_{1} \otimes n_{1}^{\prime}\right) \\
&=(\left(\epsilon_{d \otimes d^{\prime}} \otimes \mathrm{id}_{e \otimes e^{\prime}}\right) \cdot\left(\left(n_{1} \otimes n_{1}^{\prime}\right) \otimes i\left(\mathrm{id}_{d^{*} \otimes d^{\prime *}}\right) \otimes\left(n_{2} \otimes n_{2}^{\prime}\right)\right) \cdot\left(\mathrm{id}_{c \otimes c^{\prime}} \otimes \eta_{d \otimes d^{\prime}}\right) \\
&=(\left.\left(\epsilon_{d} \otimes \mathrm{id}_{e \otimes I \otimes e^{\prime}}\right) \circ\left(\mathrm{id}_{d} \otimes \sigma_{I, d^{*} \otimes e} \otimes \mathrm{id}_{e^{\prime}}\right)\right) \\
& \cdot\left[n_{1} \otimes\left(\epsilon_{d^{\prime}} \cdot\left(n_{1}^{\prime} \otimes i\left(\mathrm{id}_{d^{\prime *}}\right)\right)\right) \otimes\left(\left(i\left(\mathrm{id}_{d *}\right) \otimes n_{2}\right) \cdot \eta_{d}\right) \otimes n_{2}^{\prime}\right] \\
& \cdot\left(\left(\mathrm{id}_{c} \otimes \sigma_{I, c^{\prime} \otimes d^{\prime *}} \otimes \mathrm{id}_{d^{\prime}}\right) \circ\left(\mathrm{id}_{c \otimes I \otimes c^{\prime}} \otimes \eta_{d^{\prime}}\right)\right) \\
&=\left(\epsilon_{d} \otimes \mathrm{id}_{e \otimes I \otimes e^{\prime}}\right) \cdot\left[n_{1} \otimes\left(\left(i\left(\mathrm{id}_{d *}\right) \otimes n_{2}\right) \cdot \eta_{d}\right) \otimes\left(\epsilon_{d^{\prime}} \cdot\left(n_{1}^{\prime} \otimes i\left(\mathrm{id}_{d^{\prime *}}\right)\right)\right) \otimes n_{2}^{\prime}\right] \\
& \quad \cdot\left(\mathrm{id}_{c \otimes I \otimes c^{\prime}} \otimes \eta_{d^{\prime}}\right)
\end{aligned}
$$




$$
\begin{aligned}
= & \left(\epsilon_{d} \otimes \mathrm{id}_{e} \otimes \epsilon_{d^{\prime}} \otimes \mathrm{id}_{e^{\prime}}\right) \cdot\left[\left(n_{1} \otimes i\left(\mathrm{id}_{d^{*}}\right) \otimes n_{2}\right) \otimes\left(n_{1}^{\prime} \otimes i\left(\mathrm{id}_{d^{\prime *}}\right) \otimes n_{2}^{\prime}\right)\right] \\
& \cdot\left(\mathrm{id}_{c} \otimes \eta_{d} \otimes \mathrm{id}_{c^{\prime}} \otimes \eta_{d^{\prime}}\right) \\
= & {\left[\left(\epsilon_{d} \otimes \mathrm{id}_{e}\right) \cdot\left(n_{1} \otimes i\left(\mathrm{id}_{d^{*}}\right) \otimes n_{2}\right) \cdot\left(\mathrm{id}_{c} \otimes \eta_{d}\right)\right] } \\
& \otimes\left[\left(\epsilon_{d^{\prime}} \otimes \mathrm{id}_{e^{\prime}}\right) \cdot\left(n_{1}^{\prime} \otimes i\left(\mathrm{id}_{d^{\prime *}}\right) \otimes n_{2}^{\prime}\right) \cdot\left(\mathrm{id}_{c^{\prime}} \otimes \eta_{d^{\prime}}\right)\right] .
\end{aligned}
$$

Thus we have shown that the multiplication $\bullet$ defines a monoid $U^{-1}(N)$.

To define $U^{-1}$ on morphisms, suppose that $M \in \mathcal{M} \operatorname{nProf}(\mathscr{C}, \mathscr{C})$ is another monoidal profunctor with unit, and that $\phi: M \rightarrow N$ is a monoidal profunctor morphism which preserves units. Then $\phi$ also preserves the canonical multiplications:

$$
\begin{aligned}
\phi\left(n_{2} \bullet n_{1}\right) & =\phi\left[\left(\epsilon_{d} \otimes \mathrm{id}_{e}\right) \cdot\left(n_{1} \otimes i\left(\mathrm{id}_{d^{*}}\right) \otimes n_{2}\right) \cdot\left(\mathrm{id}_{c} \otimes \eta_{d}\right)\right] \\
& =\left(\epsilon_{d} \otimes \mathrm{id}_{e}\right) \cdot \phi\left(n_{1} \otimes i_{M}\left(\mathrm{id}_{d^{*}}\right) \otimes n_{2}\right) \cdot\left(\mathrm{id}_{c} \otimes \eta_{d}\right) \\
& =\left(\epsilon_{d} \otimes \mathrm{id}_{e}\right) \cdot\left(\phi\left(n_{1}\right) \otimes \phi\left(i_{M}\left(\mathrm{id}_{d^{*}}\right)\right) \otimes \phi\left(n_{2}\right)\right) \cdot\left(\mathrm{id}_{c} \otimes \eta_{d}\right) \\
& \left.=\left(\epsilon_{d} \otimes \mathrm{id}_{e}\right) \cdot\left(\phi\left(n_{1}\right) \otimes i_{N}\left(\mathrm{id}_{d^{*}}\right)\right) \otimes \phi\left(n_{2}\right)\right) \cdot\left(\mathrm{id}_{c} \otimes \eta_{d}\right) \\
& =\phi\left(n_{2}\right) \bullet \phi\left(n_{1}\right)
\end{aligned}
$$

Clearly $U \circ U^{-1}=\mathrm{id}_{\operatorname{Ptd}(\mathrm{CpProf})}$. For the other direction, consider a monoid $M: \mathscr{C} \rightarrow \mathscr{C}$ with unit $i$ and multiplication $\star$. Then the multiplication $\bullet$ defined above in fact coincides with $\star$ :

$$
\begin{aligned}
& \stackrel{c}{\rightarrow} n 1 \stackrel{d}{\rightarrow} n 2 \rightarrow e \\
& =\stackrel{\stackrel{c}{\rightarrow} n_{1} \rightarrow d}{\rightarrow n_{2} \rightarrow \rightarrow^{e}}=\stackrel{\left.\stackrel{c}{\rightarrow} n_{1}\right]^{d}}{{ }_{d} n_{2} \rightarrow^{e}} \\
& n_{2} \star n_{1}=n_{2} \star\left[\left(\left(\epsilon_{d} \otimes \mathrm{id}_{d}\right) \circ\left(\mathrm{id}_{d} \otimes \eta_{d}\right)\right) \cdot n_{1}\right] \\
& =\left[n_{2} \cdot\left(\epsilon_{d} \otimes \mathrm{id}_{d}\right)\right] \star\left[\left(\mathrm{id}_{d} \otimes \eta_{d}\right) \cdot n_{1}\right] \\
& =\left(i\left(\epsilon_{d}\right) \otimes n_{2}\right) \star\left(n_{1} \otimes i\left(\eta_{d}\right)\right) \\
& =\left(\epsilon_{d} \otimes \mathrm{id}_{e}\right) \cdot\left[\left(i\left(\mathrm{id}_{d}\right) \otimes i\left(\mathrm{id}_{d^{*}}\right) \otimes n_{2}\right) \star\left(n_{1} \otimes i\left(\mathrm{id}_{d^{*}}\right) \otimes i\left(\mathrm{id}_{d}\right)\right)\right] \\
& \cdot\left(\mathrm{id}_{c} \otimes \eta_{d}\right) \\
& =\left(\epsilon_{d} \otimes \mathrm{id}_{e}\right) \cdot\left(n_{1} \otimes i\left(\mathrm{id}_{d^{*}}\right) \otimes n_{2}\right) \cdot\left(\mathrm{id}_{c} \otimes \eta_{d}\right) \\
& =n_{2} \bullet n_{1}
\end{aligned}
$$

Thus $U^{-1} \circ U=\mathrm{id}_{\operatorname{Mon}(\mathrm{CpProf})}$, and $U$ is an equivalence (in fact, isomorphism) of categories.

\subsubsection{Deducing Theorem B}

We now have all the pieces in place to prove Theorem B. Recall that we use the notation $\int$ to denote the Grothendieck construction. 
Theorem B. There are equivalences of fibrations
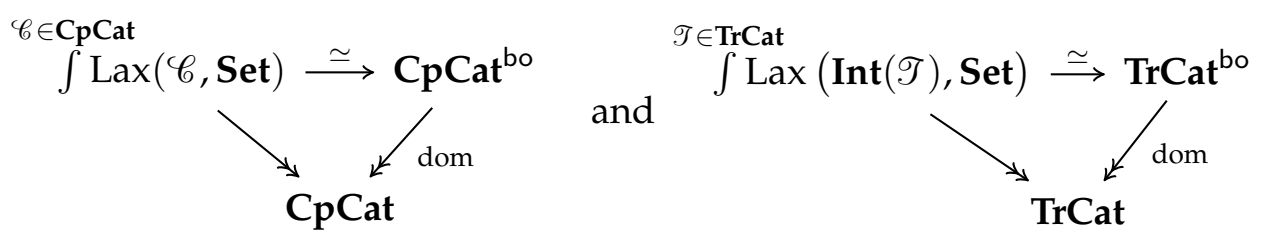

Proof. Essentially by definition, we have isomorphisms of fibrations

$$
\int \operatorname{LeCpCat}(\mathscr{C}, \text { Set }) \stackrel{\cong}{\rightrightarrows} \operatorname{Psh}(\text { CpProf }) \quad \text { and } \quad \int^{\mathscr{T} \in \operatorname{TrCat}} \operatorname{Lax}(\operatorname{Int}(\mathscr{T}), \operatorname{Set}) \stackrel{\cong}{\rightarrow} \operatorname{Psh}(\operatorname{TrProf})
$$

over CpCat and TrCat, respectively. Since CpProf and TrProf are exact by Proposition 3.3.2 and 3.3.5, we may apply Proposition 2.3.17 to get equivalences of fibrations over CpCat and TrCat:

$$
\operatorname{Mon}(\mathbb{C} \text { prof }) \stackrel{\simeq}{\rightarrow} \mathrm{CpCat}^{\text {bo }} \text { and } \operatorname{Mon}(\text { TrProf }) \stackrel{\simeq}{\rightarrow} \operatorname{TrCat}^{\text {bo }}
$$

It then suffices to prove that there are equivalences of fibrations over CpCat and TrCat:

$$
\operatorname{CPsh}(\mathbb{C} \text { pProf }) \stackrel{\simeq}{\rightarrow} \operatorname{Mon}(\operatorname{CpProf}) \text { and } \operatorname{CPsh}(\mathbb{T} \text { rProf }) \stackrel{\simeq}{\rightarrow} \operatorname{Mon}(\mathbb{T} \text { rProf })
$$

the first of which is a direct consequence of Propositions 3.4.8 and 3.4.9.

For the second equivalence, we have that Int: TrProf $\rightarrow \mathbb{C}$ pProf is a local equivalence, and it preserves the terminal object. Thus using Lemma 2.2.3 and Lemma 3.4.2 we construct the desired equivalence CPsh(TrProf $) \rightarrow$ Mon(TrProf) as the pullback along Int of the equivalence $\mathbf{C P s h}(\mathbb{C}$ prof $) \rightarrow \operatorname{Mon}(\mathbb{C}$ Prof $)$.

\subsection{Objectwise-freeness}

If we momentarily denote the free traced category on a set $\mathcal{O}$ as $F(\mathcal{O})$, a corollary of Theorem B is an isomorphism $\operatorname{Lax}(\operatorname{Int}(F(\mathcal{O}))$, Set $) \cong \operatorname{TrCat}_{F(\mathcal{O}) /}^{\text {bo }} \cong \operatorname{TrCat}_{\mathcal{O}}$, where $\operatorname{TrCat}_{\mathcal{O}}$ is the category (defined in the introduction) of traced categories whose monoid of objects is free on the set $\mathcal{O}$. This was called Theorem 0 in the introduction. Our goal in the present section is to prove Theorem A, which allows the set $\mathcal{O}$ to vary. In order to handle the cases of traced and compact categories uniformly, we first formalize what it means for an object in a general exact equipment to itself be objectwise-free.

Consider an exact equipment $\mathbb{D}$, and let dom: $\mathbb{D}_{0}^{\text {bo }} \rightarrow \mathbb{D}_{0}$ denote the domain fibration. Suppose we are given an adjunction to a category $\mathscr{S}$ :

$$
\mathscr{S} \underset{U}{\stackrel{F}{\rightleftarrows}} \mathbb{D}_{0}
$$

Let $T=U F$ be the monad on $\mathscr{S}$ corresponding to this adjunction, and write $\mathscr{I}_{T}$ for the Kleisli category for $T$, i.e. the full subcategory of free objects $F s$ in $\mathbb{D}_{0}$. Let $k_{T}: \mathscr{I}_{T} \rightarrow \mathbb{D}_{0}$ 
denote the inclusion, and define $k_{T}^{\text {bo }}$ to be the strict pullback of $k_{T}$ along dom:

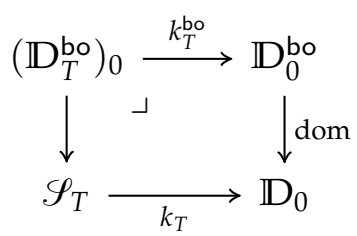

Definition 3.5.1. The fully faithful functors $k_{T}: \mathscr{S}_{T} \rightarrow \mathbb{D}_{0}$ and $k_{T}^{\text {bo }}:\left(\mathbb{D}_{T}^{\text {bo }}\right)_{0} \rightarrow \mathbb{D}_{0}^{\text {bo }}$ induce equipments $\mathbb{D}_{T}:=k_{T}^{*} \mathbb{D}$ and $\mathbb{D}_{T}^{\text {bo }}:=\left(k_{T}^{\text {bo }}\right)^{*} \mathbb{D}^{\text {bo }}$ (as in Definition 2.1.5), as well as fully faithful local equivalences, which we denote

$$
\varphi_{T}: \mathbb{D}_{T} \rightarrow \mathbb{D} \quad \text { and } \quad \varphi_{T}^{\text {bo }}: \mathbb{D}_{T}^{\text {bo }} \rightarrow \mathbb{D}^{\text {bo }} .
$$

We can now apply the previous abstract definition to define equipments of objectwisefree monoidal, compact, and traced categories, preparing for the proof of Theorem A. Consider the free-forgetful adjunctions ${ }^{6}$

$$
\text { Set } \underset{U_{M}}{\stackrel{F_{M}}{\rightleftarrows}} \text { MnCat Set } \underset{U_{C}}{\stackrel{F_{C}}{\rightleftarrows}} \text { CpCat } \text { Set } \underset{U_{T}}{\stackrel{F_{T}}{\rightleftarrows}} \text { TrCat }
$$

and write $\mathbf{T}_{M}, \mathbf{T}_{C}$, and $\mathbf{T}_{T}$ for the corresponding monads on Set. Note that $\mathbf{T}_{M}$ and $\mathbf{T}_{\mathrm{T}}$ are both isomorphic to the free monoid monad, while $\mathbf{T}_{\mathrm{C}}$ is isomorphic to the free monoid-with-involution monad. ${ }^{7}$ Following Definition 3.5.1, we have equipments:

$$
\mathbb{F} \text { MnProf }:=\mathbb{M n P r o f}_{\mathrm{T}_{\mathrm{M}}} \quad \mathbb{F} \text { CpProf }:=\operatorname{CpProf}_{\mathrm{T}_{\mathrm{C}}} \quad \mathbb{F T r P r o f}:=\operatorname{TrProf}_{\mathrm{T}_{\mathrm{T}}}
$$

The functor FMnProf $\rightarrow \mathbb{M n P r o f}$ is a fully faithful local equivalence, meaning it can be identified with the full sub-equipment of $\mathbb{M n P r o f}$ spanned by the monoidal categories which are free on a set; similarly for $\mathbb{F C p P r o f}$ and $\mathbb{F T r P r o f}$. We will write

$$
\begin{aligned}
\text { FMnCat } & :=\text { FMnProf }_{0}=\text { Set }_{\mathrm{T}_{\mathrm{M}}} \\
\text { FCpCat } & :=\mathbb{F C p P r o f}_{0}=\text { Set }_{\mathrm{T}_{\mathrm{C}}} \\
\text { FTrCat } & :=\mathbb{F T r P r o f}_{0}=\text { Set }_{\mathrm{T}_{\mathrm{T}}}
\end{aligned}
$$

for the vertical 1-categories. Note that each of these categories has a terminal object.

Similarly, we can define

$$
\begin{aligned}
& \text { MnFrObCat }:=\text { Vert }\left(\mathbb{M n P r o f}_{\mathrm{T}_{\mathrm{M}}}^{\mathrm{bo}}\right) \quad \text { EpFrObCat }:=\text { Vert }\left(\operatorname{CpProf}_{\mathrm{T}_{\mathrm{C}}}^{\text {bo }}\right) \\
& \mathcal{T} \text { rFrObCat }:=\text { Vert }\left(\operatorname{TrProf}_{\mathbf{T}_{\mathrm{T}}}^{\text {bo }}\right) \text {. }
\end{aligned}
$$

The functor MnProf $\mathbf{T}_{\mathbf{M}}^{\text {bo }} \rightarrow \mathbb{M n P r o f}^{\text {bo }}$ is also a fully faithful local equivalence. Hence $\mathcal{M n F r O b C a t}$ is the full sub-2-category of $\mathcal{M}$ nCat ${ }^{\text {bo }}$ spanned by those bijective-on-objects monoidal functors $\mathscr{M}^{\prime} \rightarrow \mathscr{M}$ for which $\mathscr{M}^{\prime}=\mathbf{F}_{\mathrm{M}}(\mathcal{O})$ is free on a set. Note that this is equivalent to the full sub-2-category of $\mathcal{M n C a t}$ spanned by those monoidal categories $\mathscr{M}$ which are objectwise-free, i.e. whose underlying monoid of objects is free. Likewise for $\mathcal{E p F r O b C a t}$ and $\mathcal{T}$ rFrObCat.

\footnotetext{
6 These three adjunctions in fact extend to 2-adjunctions; see Corollary A.1.4.

${ }^{7}$ Note that $\mathbf{T}_{\mathrm{M}}$ is not the free-commutative-monoid monad, even though the objects of MnCat are symmetric monoidal categories, because the symmetries are encoded by natural isomorphisms, not equalities.
} 
Remark 3.5.2. We have defined a 2-category $\mathcal{M n F r O b C a t}$ of objectwise-free monoidal categories, which are also known as (colored) PROPs (see, e.g. [8] for more on PROPs). However, the morphisms between PROPs are more restrictive than those defined above, because they must "send colors to colors". To define an equipment of PROPs, consider the functor $\mathbf{F}_{\mathrm{M}}$ : Set $\rightarrow$ MnCat and let $\mathbb{P R O P}:=\mathbf{F}_{\mathrm{M}}^{*} \mathbf{M n P r o f}$ be the induced equipment. Similarly, one can define traced and compact (colored) PROPs as $\mathbf{F}_{\mathrm{T}}^{*} \mathrm{TrProf}$ and $\mathbf{F}_{\mathrm{C}}^{*} \mathrm{C}$ pProf respectively.

Although we will not prove it here, one can prove a variant of Theorem A, namely that there are equivalences of categories

$$
\int^{\mathcal{O} \in \text { Set }}\left(\mathrm{Cob}_{/ \mathcal{O}}\right)-\mathbf{A l g} \rightarrow \text { CpPROP } \text { and } \int^{\mathcal{O} \in \text { Set }}\left(\mathrm{Cob}_{/ \mathcal{O}}\right)-\mathbf{A l g} \rightarrow \text { TrPROP. }
$$

See [10] for another approach to compact PROPs.

The proof of Theorem A depends on connecting monoids in FCpProf and FTrProf to $\mathcal{C p F r O b C a t}$ and $\mathcal{T}$ rFrObCat. We first prove this connection in the abstract setting, then specialize to the cases of interest in Corollary 3.5.4.

Proposition 3.5.3. With the setup as in Definition 3.5.1, suppose also that $\mathbb{D}$ is exact and has local reflexive coequalizers. There is a commutative diagram of equipments, in which the vertical functors are equivalences and the horizontal functors are local equivalences:

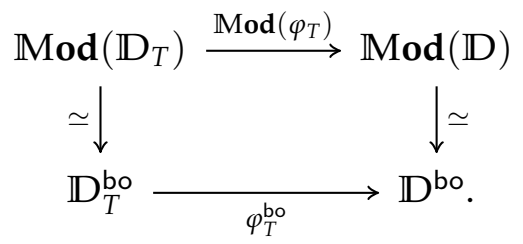

Suppose moreover that $U($ bo $) \subseteq$ iso $(\mathscr{S})$, the set of isomorphisms in $\mathscr{S}$. Then the following composite is a fully faithful local equivalence:

$$
\operatorname{Mod}\left(\mathbb{D}_{T}\right) \stackrel{\operatorname{Mod}\left(\varphi_{T}\right)}{\longrightarrow} \operatorname{Mod}(\mathbb{D}) \stackrel{\langle-\rangle}{\longrightarrow} \mathbb{D} .
$$

Proof. By Lemma 2.2.5, $\operatorname{Mod}\left(\varphi_{T}\right): \operatorname{Mod}\left(\mathbb{D}_{T}\right) \rightarrow \mathbb{M o d}(\mathbb{D})$ is a fully faithful local equivalence. The remainder of the first claim follows from Theorem 2.3.18 and the definition of $\mathbb{D}_{T}^{\text {bo }}$.

For the second claim, assume $U($ bo $) \subseteq$ iso $(\mathscr{S})$. From Theorem 2.3.18 and the first part of the proposition, it suffices to consider the composition

$$
\mathbb{D}_{T}^{\text {bo }} \stackrel{\varphi_{T}^{\text {bo }}}{\longrightarrow} \mathbb{D}^{\text {bo }} \stackrel{\text { cod }}{\longrightarrow} \mathbb{D} \text {. }
$$

By definition, both $\varphi_{T}^{\text {bo }}$ and cod are local equivalences, hence the composition is also.

To see that $\left(\operatorname{cod} \varphi_{T}^{\text {bo }}\right)_{0}$ is fully faithful, consider a pair of objects $p: F s \rightarrow D$ and $p^{\prime}: F s^{\prime} \rightarrow D^{\prime}$ in $\left(\mathbb{D}_{T}^{\text {bo }}\right)_{0}$, and a vertical morphism $f: D \rightarrow D^{\prime}$ in $\mathbb{D}_{0}$. In the square

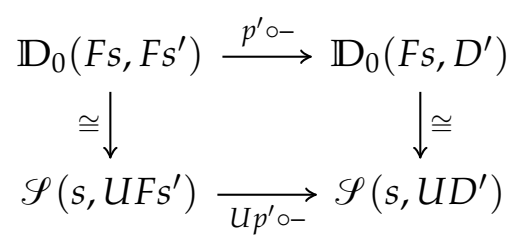


which commutes by naturality of the adjunction bijection, the bottom function is a bijection since $U\left(p^{\prime}\right)$ is an isomorphism for any $p^{\prime} \in$ bo. Hence the top function is a bijection, which shows that there exists a unique lift of $f$ to a morphism in $\left(\mathbb{D}_{T}^{\text {bo }}\right)_{0}$ :

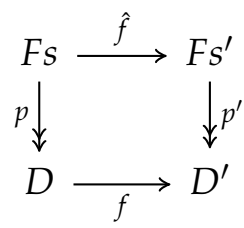

as desired.

Corollary 3.5.4. There are fully faithful local equivalences of equipments, in the left column, and equivalences of 2-categories, in the right column:

$$
\begin{aligned}
& \operatorname{Mod}(\mathbb{F} M n P r o f) \rightarrow \mathbb{M n P r o f} \quad \mathcal{M} \text { on }(\mathbb{F M n P r o f}) \simeq \mathcal{M} \text { nFrObCat } \\
& \operatorname{Mod}(\mathbb{F T r P r o f}) \rightarrow \mathbb{T} \text { rProf } \quad \mathcal{M} \text { Ton }(\text { FTrProf }) \simeq \mathcal{T} \text { rFrObCat } \\
& \operatorname{Mod}(\mathbb{F C} \text { pProf }) \rightarrow \text { CpProf } \quad \mathcal{M} \text { on }(\text { FCpProf }) \simeq \text { epFrObCat. }
\end{aligned}
$$

Proof. The left column comes from the second part of Proposition 3.5.3, while the right column follows by applying Vert to the equivalence $\operatorname{Mod}\left(\mathbb{D}_{T}\right) \simeq \mathbb{D}_{T}^{\text {bo }}$ in the first part of Proposition 3.5.3.

The last piece needed for the proof of Theorem $\mathrm{A}$ is to show that the equivalence between copresheaves and monoids in CpProf and TrProf proven in Section 3.4 restricts to FCpProf and FTrProf.

Lemma 3.5.5. There are equivalences of fibrations

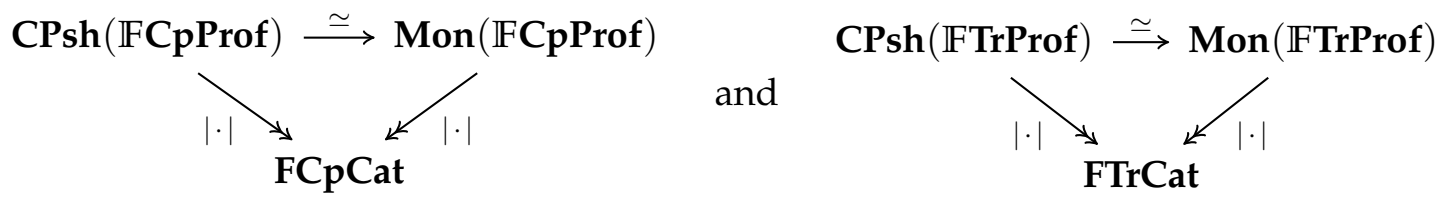

Proof. The equipment functor $\varphi_{\mathrm{C}}: \mathbb{F C p P r o f} \rightarrow \mathbb{C}$ PProf (resp. $\varphi_{\mathrm{T}}:$ FTrProf $\rightarrow$ TrProf) is by definition a local equivalence, and it preserves terminal objects in the vertical category. Thus using Lemma 2.2.3 and Lemma 3.4.2 we construct the desired equivalence $\operatorname{CPsh}(\mathbb{F C p P r o f}) \rightarrow \operatorname{Mon}(\mathbb{F C p P r o f})$ as the pullback along $\varphi_{\mathrm{C}}$ of the equivalence $\operatorname{CPsh}(\mathbb{C}$ pProf $) \rightarrow$ Mon(CpProf) from (34), and similarly for the traced case.

Finally, we are ready to prove Theorem A.

Theorem A. There are equivalences of 1-categories

$$
\int^{\mathcal{O} \in \operatorname{Set}_{\mathrm{T}_{\mathrm{C}}}}\left(\mathrm{Cob}_{/ \mathcal{O}}\right)-\mathrm{Alg} \rightarrow \text { CpFrObCat and } \int^{\mathcal{O} \in \operatorname{Set}_{\mathrm{T}_{\mathrm{T}}}}\left(\mathrm{Cob}_{/ \mathcal{O}}\right)-\mathrm{Alg} \rightarrow \text { TrFrObCat. }
$$


Proof. First note that, essentially by definition (as well as the fact that $\mathbf{C o b} / \mathcal{O}$ is the free compact category on the set $\mathcal{O}$; see $[13,2])$, there are isomorphisms of fibrations
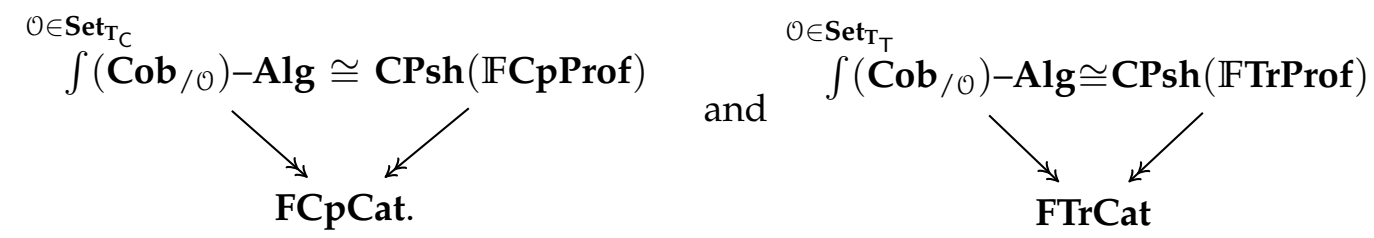

By Lemma 3.5.5, we have equivalences of 1-categories

$$
\operatorname{CPsh}(\mathbb{F C p P r o f}) \simeq \operatorname{Mon}(\mathbb{F C p P r o f}) \quad \text { and } \quad \operatorname{CPsh}(\mathbb{F T r P r o f}) \simeq \operatorname{Mon}(\mathbb{F T r P r o f})
$$

The result now follows from Corollary 3.5.4, which provides equivalences of 2-categories:

$$
\mathcal{M} \operatorname{Mon}(\mathbb{F C p P r o f}) \simeq \mathcal{C p F r O b C a t} \text { and } \mathcal{M} \operatorname{Mon}(\mathbb{F T r P r o f}) \simeq \mathcal{T} \text { rFrObCat }
$$

\subsection{A traceless characterization of $\widetilde{\mathcal{T} \text { rCat }}$}

In this final section, we briefly record a construction of a 2-category bi-equivalent to the 2category $\widetilde{\mathcal{T} \text { rCat }}$ of traced categories with strong functors between them. A distinguishing feature of this construction is that it makes no mention of a trace operation, nor anything akin to the usual traced category axioms. It is a direct consequence of the machinery used to prove our main theorems, and is - to the best of our knowledge-a new result.

The forgetful functors between the categories of structured monoidal categories commute with the underlying set functors, i.e. the following diagram commutes:

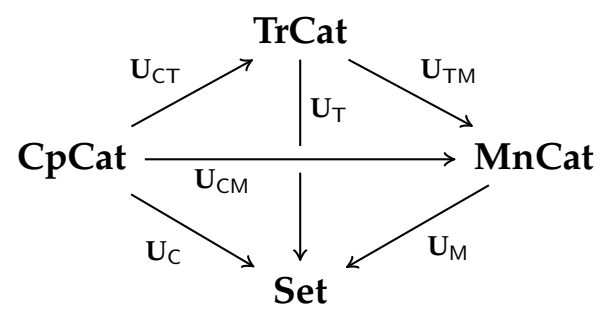

Because the functor $\mathbf{U}_{\mathrm{CM}}$ : CpCat $\rightarrow$ MnCat commutes with the right adjoints of the adjunctions to Set, i.e. $\mathbf{U}_{M} \mathbf{U}_{C M}=\mathbf{U}_{C}$, it induces a monad morphism $\alpha: \mathbf{T}_{M} \rightarrow \mathbf{T}_{C}$ (i.e. a natural transformation $\alpha: \mathbf{T}_{\mathrm{M}} \rightarrow \mathbf{T}_{\mathrm{C}}$ compatible with the units and multiplications), given by the composition of the natural transformations

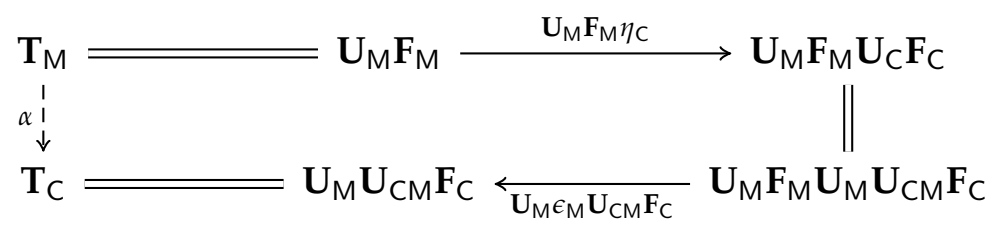

The component $\alpha_{\mathcal{O}}$ of this transformation is simply the evident inclusion of the free monoid on a set $\mathcal{O}$ into the free monoid-with-involution on $\mathcal{O}$. The monad map $\alpha$ induces a functor between the Kleisli categories:

$$
\mathbf{F}_{\mathrm{MC}}: \operatorname{Set}_{T_{\mathrm{M}}} \rightarrow \operatorname{Set}_{T_{\mathrm{C}}} .
$$


Because the monads $\mathbf{T}_{\mathrm{M}}$ and $\mathbf{T}_{\mathrm{T}}$ are in fact isomorphic, we have

$$
\text { FTrCat }=\text { Set }_{T_{\mathrm{T}}} \cong \operatorname{Set}_{\mathrm{T}_{\mathrm{M}}}=\text { FMnCat. }
$$

The following proposition defines the 2-category $\widetilde{\mathcal{T} \text { rCat }}$ of traced categories, and strong functors, purely in terms of $\mathbb{C}$ pProf, MnProf, and the adjunctions $\mathbf{F}_{\mathbf{M}} \dashv \mathbf{U}_{\mathbf{M}}$ and $\mathbf{F}_{\mathrm{C}} \dashv \mathbf{U}_{\mathrm{C}}$. In particular, it does not involve any explicit mention of the trace structure defined in [12]. However, it does use the main result of the appendix, Corollary A.3.2.

Proposition 3.6.1. Consider the functor FMnCat $\stackrel{\mathrm{F}_{\mathrm{MC}}}{\longrightarrow} \mathrm{FCpCat} \stackrel{{ }_{\mathrm{c}}}{\rightarrow} \mathrm{CpCat}$ and the induced equipment $\mathbb{F}:=\left(k_{\mathrm{C}} \circ \mathbf{F}_{\mathrm{MC}}\right)^{*}(\mathbf{C}$ Prof $)$. There is a fully faithful local equivalence $\mathbb{M o d}(\mathbb{F}) \rightarrow$ $\mathbb{T r P r o f}$ and an equivalence of 2 -categories $\mathcal{M} \operatorname{Mon}(\mathbb{F}) \simeq \widehat{\mathcal{T} \text { rCat. }}$

Proof. By combining the definitions of FTrProf and TrProf, it is easy to see that the following square is a pullback:

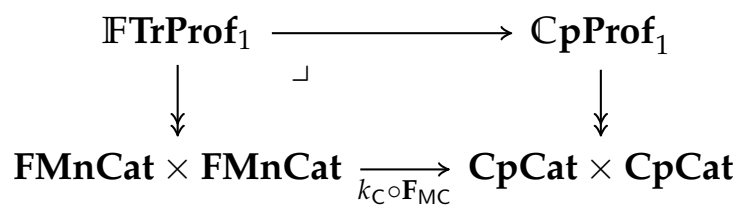

Thus we have an equivalence $\mathbb{F} \simeq \mathbb{F}$ TrProf, and the result follows by Corollary 3.5.4 and Corollary A.3.2.

\section{A Appendix}

This section is mostly independent from the rest of the paper. It is really only used to prove Corollary A.3.2, the three biequivalences

$$
\mathcal{M n F r O b C a t} \rightarrow \widetilde{\mathcal{M n C a t}} \quad \mathcal{T} \text { rFrObCat } \rightarrow \widetilde{\mathcal{T} \text { rCat }} \quad \text { epFrObCat } \rightarrow \widetilde{\text { epCat. }}
$$

Here, $\mathcal{M}$ nFrObCat (resp. $\mathcal{T}$ rFrObCat and $\mathcal{C p F r O b C a t )}$ is the 2-category of objectwisefree monoidal (resp. traced and compact) categories and strict functors between them (see Section 3.5), whereas $\widetilde{\mathcal{M} \text { Cat }}$ (resp. $\widetilde{\mathcal{T} \text { rCat }}$ and $\widetilde{\text { CpCat }}$ ) is the 2-category of monoidal (resp. traced and compact) categories with arbitrary objects and strong functors between them. This result will not be new to experts, but we found it difficult to find in the literature.

\section{A.1 Arrow objects and mapping path objects}

Definition A.1.1. Let $a$ be an object in a 2-category $\mathcal{C}$. An arrow object of $a$ is an object $a^{2}$ together with a diagram

$$
a^{2} \underset{\frac{\operatorname{cod}}{\Downarrow_{k}}}{\operatorname{dom}} a
$$


which is universal among such diagrams: any diagram as on the left below factors uniquely as on the right

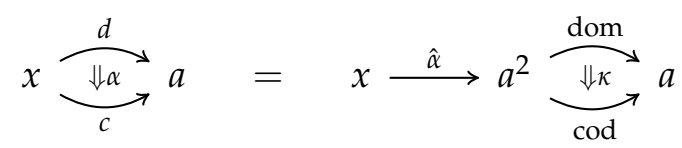

Moreover, given a commutative square in $\mathcal{C}(x, a)$, i.e. another $d^{\prime}: x \rightarrow a, c^{\prime}: x \rightarrow a$, $\alpha^{\prime}: d^{\prime} \Rightarrow c^{\prime}$ as on the left above, and 2-cells $\beta: d \Rightarrow d^{\prime}$ and $\gamma: c \Rightarrow c^{\prime}$ such that $\alpha^{\prime} \circ \beta=$ $\gamma \circ \alpha$, there is a unique $(\beta, \gamma): \hat{\alpha} \Rightarrow \hat{\alpha}^{\prime}$ such that $\operatorname{dom}(\beta, \gamma)=\beta$ and $\operatorname{cod}(\beta, \gamma)=\gamma$.

We say that $\mathcal{C}$ has arrow objects if an arrow object $a^{2}$ exists for each object $a \in \mathscr{C}$.

Example A.1.2. The 2-categories $\mathcal{C a t}, \mathcal{C a t} \cong, \widetilde{\mathcal{M n C a t}}, \widetilde{\mathcal{T} \text { rCat }}$, and $\widetilde{\mathcal{E p C a t}}$ have arrow objects. Clearly for an object $\mathscr{A} \in \mathcal{C}$ at, the usual arrow category $\mathscr{A}^{2}$ of arrows and commutative squares, has the necessary universal property. Similarly, the arrow category of $\mathscr{A}$ in Cat $\cong$ is the category whose objects are isomorphisms in $\mathcal{A}$, and whose morphisms are commutative squares (in which the other morphisms need not be isomorphisms).

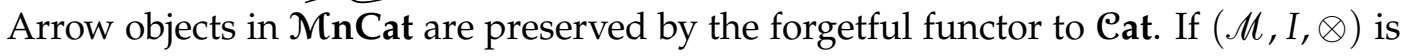
a monoidal category then the arrow object $\mathscr{M}^{2}$ (in Cat) has a natural monoidal product

$$
\mathscr{M}^{2} \times \mathscr{M}^{2} \cong(\mathscr{M} \times \mathscr{M})^{2} \stackrel{\otimes^{2}}{\longrightarrow} \mathscr{M}^{2},
$$

and monoidal unit given by the identity $\operatorname{map} \mathrm{id}_{I}$ on the unit of $\mathcal{M}$. The maps dom, cod: $\mathcal{M}^{2} \rightarrow \mathcal{M}$ are strict monoidal functors, and the transformation $\kappa:$ dom $\rightarrow$ cod is monoidal as well. Suppose given a diagram of strong monoidal functors:

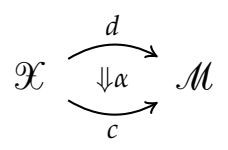

The universal properties of the arrow object $\mathscr{M}^{2}$ in Cat guarantee that the induced functor $\hat{\alpha}: \mathscr{X} \rightarrow \mathscr{M}^{2}$ is strong monoidal. Note that if $d, c$ are strict monoidal functors then $\hat{\alpha}$ will be as well.

The 2-category $\mathrm{CpCat}$ also has arrow objects, and they are preserved by the forgetful functor $\widehat{\mathcal{E p C a t}} \rightarrow$ Cat $\cong$. Recall from Section 3.1 that every natural transformation between compact categories is an isomorphism. Thus for a compact category $\mathscr{C}$, the arrow category $\mathscr{C}^{2}$ has as objects the isomorphisms $a \cong b$ in $\mathscr{C}$, and as morphisms the commuting squares. This is compact: the dual of $f: a \rightarrow b$ is $\left(f^{-1}\right)^{*}: a^{*} \rightarrow b^{*}$.

The 2-morphisms between traced categories are also defined to be isomorphisms

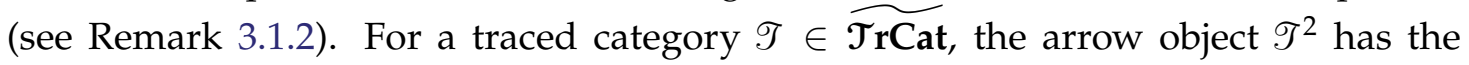
isomorphisms in $\mathscr{T}$ as objects and commuting squares as morphisms; i.e. here too arrow objects are preserved by the 2-functor $\widetilde{\mathcal{T} \text { rCat }} \rightarrow$ Cat $\cong$. To see the traced structure of $\mathscr{T}^{2}$, suppose given objects $a: A \stackrel{\cong}{\rightarrow} A^{\prime}, b: B \stackrel{\cong}{\rightarrow} B^{\prime}$, and $u: U \stackrel{\cong}{\rightarrow} U^{\prime}$, as well as a morphism 
$(f, g): a \otimes u \rightarrow b \otimes u$ as in the diagram to the left
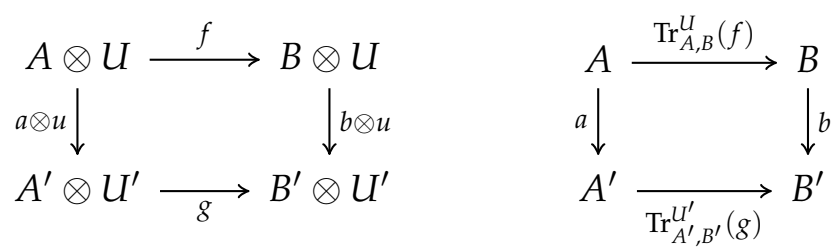

Composing with $\mathrm{id}_{B^{\prime}} \otimes u^{-1}$, we have

$$
\left(\mathrm{id}_{B^{\prime}} \otimes u^{-1}\right) \circ(b \otimes u) \circ f=\left(\mathrm{id}_{B^{\prime}} \otimes u^{-1}\right) \circ g \circ(a \otimes u)
$$

as morphisms $A \otimes U \rightarrow B^{\prime} \otimes U$. The commutativity of the right-hand diagram in (40) follows from this equation and the axioms of traced categories [12].

Lemma A.1.3. Let $R: \mathcal{C} \rightarrow \mathcal{D}$ be a 2-functor, and suppose that $\mathcal{C}$ has arrow objects. Then $R$ has a left 2-adjoint if and only if $R$ has a left 1-adjoint and $R$ preserves arrow objects.

Proof. First suppose $R$ has a left 1-adjoint $L$ and preserves arrow objects. We want to show that given morphisms $f, g: D \rightarrow R C$ in $\mathcal{D}$ and a 2-cell $\alpha: f \Rightarrow g$, there is a unique $\alpha^{\prime}$ in $\mathrm{C}$ such that $R\left(\alpha^{\prime}\right) \eta_{D}=\alpha$. From the 1-adjunction, we know there are unique $f^{\prime}, g^{\prime}: L D \rightarrow C$ such that $R f^{\prime} \circ \eta_{D}=f$ and $R g^{\prime} \circ \eta_{D}=g$. Using the arrow object $R\left(C^{2}\right)=(R C)^{2}$, there is a unique morphism $\hat{\alpha}: D \rightarrow R C^{2}$ such that $\kappa_{R C} \hat{\alpha}=\alpha$. Using the 1 -adjunction again, there is a unique $\hat{\alpha}^{\prime}: L D \rightarrow C^{2}$ such that $R \hat{\alpha}^{\prime} \circ \eta_{D}=\hat{\alpha}$. Finally, we let $\alpha^{\prime}:=\kappa_{C} \hat{\alpha}^{\prime}$, and check

$$
R\left(\alpha^{\prime}\right) \eta_{D}=R\left(\kappa_{C}\right) R\left(\hat{\alpha}^{\prime}\right) \eta_{D}=\kappa_{R C} \hat{\alpha}=\alpha .
$$

It is clear that this $\alpha^{\prime}$ is the unique such 2-cell.

Conversely, it is easy to check that if $R$ has a left 2-adjoint, then $R$ preserves arrow objects (right adjoints preserve limits).

The following result was promised above; see (36) and footnote 6.

Corollary A.1.4. There are 2-adjunctions

$$
\mathrm{F}_{\mathrm{M}}: \text { eat } \leftrightarrows \widetilde{\mathcal{M} \text { nCat }}: \mathrm{U}_{\mathrm{M}} \quad \mathrm{F}_{\mathrm{T}}: \text { eat } \cong \widetilde{\mathcal{T r C a t}}: \mathrm{U}_{\mathrm{T}} \quad \mathrm{F}_{\mathrm{C}}: \text { Cat } \cong \leftrightarrows \widetilde{\text { epCat }}: \mathrm{U}_{\mathrm{C}}
$$

that extend the 1-adjunctions constructed in [2].

Proof. Let $R$ be either $\mathbf{U}_{\mathrm{M}}, \mathbf{U}_{\mathrm{T}}$, or $\mathbf{U}_{\mathrm{C}}$. Its underlying 1-functor has a left adjoint, constructed in [2]. We showed in Example A.1.2 that $\widetilde{\mathcal{M n C a t}}, \widetilde{\mathcal{T} \text { rCat }}$, and $\widetilde{\text { epCat }_{\text {pave }}}$ arrow objects, which are preserved by $R$. The result follows by Lemma A.1.3.

Definition A.1.5. Let $f: a \rightarrow b$ be a morphism in a 2-category C. A mapping path object of $f$ is an object $P(f)$ together with a diagram

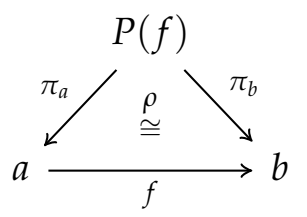


where $\rho$ is an isomorphism, which is universal among such diagrams: any diagram as on the left below, in which $\alpha$ is an isomorphism, factors uniquely as on the right

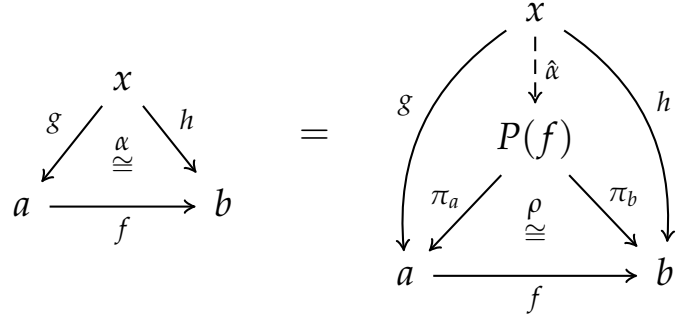

Moreover, given another $g^{\prime}: x \rightarrow a, h^{\prime}: x \rightarrow b, \alpha^{\prime}: f g^{\prime} \cong h^{\prime}$ as on the left above, and isomorphisms $\beta: g \cong g^{\prime}$ and $\gamma: h \cong h^{\prime}$ such that $\alpha^{\prime} \circ f \beta=\gamma \circ \alpha$, there is a unique isomorphism $(\beta, \gamma): \hat{\alpha} \cong \hat{\alpha}^{\prime}$ such that $\pi_{a}(\beta, \gamma)=\beta$ and $\pi_{b}(\beta, \gamma)=\gamma$.

We say that $\mathcal{C}$ has mapping path objects if a mapping path object $P(f)$ exists for each morphism $f: a \rightarrow b$ in $\mathscr{C}$.

Example A.1.6. The 2-categories Cat, eat $\cong \widetilde{\mathcal{M n C a t}}, \widetilde{\mathcal{T} \text { rCat }}$, and $\widetilde{\text { epCat }}$ have mapping path objects. For a morphism $F: \mathscr{A} \rightarrow \mathscr{B}$ in Cat, the mapping path category $P(F)$ is a cousin to the comma category $\left(F \downarrow \mathrm{id}_{\mathscr{B}}\right)$ : the objects are triples

$$
\mathrm{Ob}(P(F)):=\{(A, B, i) \mid A \in \mathrm{Ob}(\mathscr{A}), B \in \mathrm{Ob}(\mathscr{B}), i: F(A) \stackrel{\cong}{\rightarrow} B \text { is an isomorphism }\}
$$

and a morphism $(A, B, i) \rightarrow\left(A^{\prime}, B^{\prime}, i^{\prime}\right)$ in $P(F)$ consists of a pair of morphisms $A \rightarrow A^{\prime}$ in $\mathscr{A}$ and $B \rightarrow B^{\prime}$ in $\mathscr{B}$ such that the evident diagram commutes. The 2-category $\mathcal{C} \cong$ has exactly the same mapping path objects as Cat.

The mapping path object of a strong functor $F: \mathscr{A} \rightarrow \mathscr{B}$ between monoidal, traced, or compact categories exists and is preserved by the forgetful functors to $\mathcal{C}$ at and $\mathbf{C a t} \cong$. In the monoidal case, the mapping path object $P(F)$ of the functor between underlying categories has a canonical monoidal structure, e.g.,

$$
(A, B, i) \otimes\left(A^{\prime}, B^{\prime}, i^{\prime}\right):=\left(A \otimes A^{\prime}, B \otimes B^{\prime},\left(i \otimes i^{\prime}\right) \circ \mu_{A, A^{\prime}}^{-1}\right)
$$

where $\mu_{A, A^{\prime}}$ is the coherence isomorphism for $F$. The projection functors $A \stackrel{\pi_{A}}{\longleftarrow} P(F) \stackrel{\pi_{B}}{\longrightarrow}$ $B$ are strict. Given a diagram

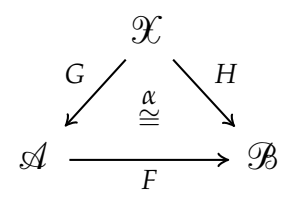

in which $G$ and $H$ are strong (resp. strict) monoidal functors, the induced functor $\hat{\alpha}: \mathscr{X} \rightarrow P(F)$, given on objects by $x \mapsto\left(G(x), H(x), \alpha_{x}\right)$, will be strong (resp. strict) as well.

If $\mathscr{A}$ and $\mathscr{B}$ are traced categories and $F$ is a traced functor, one obtains a canonical trace structure on the monoidal category $P(F)$ in a manner similar to that shown in Example A.1.2. If $\mathscr{A}$ and $\mathscr{B}$ are compact categories, then the mapping path monoidal category $P(F)$ is naturally compact: the dual of $(A, B, i)$ is $\left(A^{*}, B^{*},\left(i^{-1}\right)^{*}\right)$. 
Remark A.1.7. The arrow objects and mapping path objects for the 2-categories $\widetilde{\mathcal{M n C a t}}$ $\widetilde{\mathcal{T} \text { rCat, }}$ and $\widetilde{\text { epCat }}$ were discussed in Examples A.1.2 and A.1.6. Each has a notion of cone, in fact a certain weighted limit cone in Cat, though we will not discuss that notion here. We mentioned in passing that the structure morphisms for that cone are strict monoidal functors and that they "preserve and jointly detect" strictness in the sense of Definition A.2.1 below. In particular, the 2-categories $\mathcal{M}$ nCat, $\mathcal{T}$ rCat, and $\mathcal{E p C a t}$ also have arrow objects and mapping path objects, and the inclusions of strict-into-strong (e.g. MnCat $\rightarrow \widehat{\mathcal{M n C a t}}$ ) preserve them. Looking back at Examples A.1.2 and A.1.6, we see that the forgetful functors

$$
\mathrm{U}_{\mathrm{M}}: \mathcal{M n C a t} \rightarrow \text { Cat } \quad \mathrm{U}_{\mathrm{T}}: \mathcal{T} \text { rCat } \rightarrow \text { Cat } \cong \quad \mathrm{U}_{\mathrm{C}}: \mathcal{C} \text { pat } \rightarrow \text { Cat } \cong
$$

preserve arrow objects and mapping path objects.

Definition A.1.8. A morphism $f: a \rightarrow b$ in a 2-category $\mathcal{C}$ is fully faithful if the functor $f^{*}: \mathcal{C}(x, a) \rightarrow \mathfrak{C}(x, b)$, induced by composition with $f$, is fully faithful for every $x$. That is, $f$ is fully faithful if, for every diagram

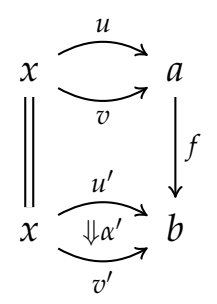

such that $f u=u^{\prime}$ and $f v=v^{\prime}$, there exists a unique $\alpha: u \Rightarrow v$ such that $f \alpha=\alpha^{\prime}$.

A morphism $f: a \rightarrow b$ in a 2-category $\mathcal{C}$ is bijective-on-objects if it is left orthogonal to every fully faithful morphism.

Definition A.1.9. Say that a morphism $f: a \rightarrow b$ in a 2-category $\mathcal{C}$ is a surjective equivalence if it can be extended to an adjoint equivalence $g \dashv f$ in which the unit is the identity. That is, there is a morphism $g: b \rightarrow a$ and 2-cell $\epsilon: g f \cong 1_{a}$ such that $f g=1_{b}$, $\epsilon g=1_{g}$, and $f \epsilon=1_{f}$.

Lemma A.1.10. Let $f: a \rightarrow b$ and $g: b \rightarrow a$ be morphisms in a 2-category such that $f g=1_{b}$. Then $f$ (together with $g$ ) is a surjective equivalence if and only if $f$ is fully faithful in the sense of Definition A.1.8.

Proof. Suppose $g \dashv f$ is a surjective equivalence. Then for any $x, f^{*}: \mathcal{C}(x, a) \rightarrow \mathcal{C}(x, b)$ is an equivalence of categories, hence fully faithful. Thus $f$ is fully faithful.

Conversely suppose $f$ is fully faithful. Then because $f g f=f=f 1_{a}$, there is a unique $\epsilon: g f \Rightarrow 1_{a}$ such that $f \epsilon=1_{f}$. It is easy to check that $\epsilon$ is an isomorphism, and that $\epsilon g=1_{g}$.

Lemma A.1.11. For any morphism $f: a \rightarrow b$ with a mapping path object $P(f)$, the projection $\pi_{a}: P(f) \rightarrow a$ is a surjective equivalence, hence fully faithful. 
Proof. By the universal property of $P(f)$ there is a unique morphism $s: a \rightarrow P(f)$ such that

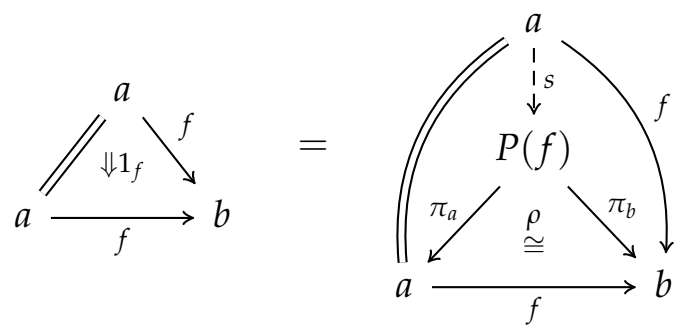

Because $\pi_{a} s \pi_{a}=\pi_{a}$ and $\pi_{b} s \pi_{a}=f \pi_{a} \cong \pi_{b}$, we can use the 2-dimensional universality of $P(f)$ to obtain a unique isomorphism $\epsilon: s \pi_{a} \cong 1_{P(f)}$ such that $\pi_{a} \epsilon=1_{\pi_{a}}$ and $\pi_{b} \epsilon=\rho$. By 2-dimensional universality once more, we obtain $\epsilon S=1_{s}$ from the following facts

$$
\pi_{a} \epsilon S=1_{\pi_{a}} s=\pi_{a} 1_{s} \quad \text { and } \quad \pi_{b} \epsilon S=\rho s=1_{f}=\pi_{b} 1_{s} .
$$

It follows from Lemma A.1.10 that $\pi_{a}$ is fully faithful.

\section{A.2 Strict vs. strong morphisms}

Between monoidal categories, there are several notions of functor: strict, strong, lax, and colax. While researchers tend to be most interested in the 2-category $\widehat{\mathcal{M n C a t}}$ of monoidal categories and strong functors, and similarly $\widetilde{\mathcal{T} \text { rCat }}$ and $\widetilde{\mathcal{E} \text { pCat, the strict }}$ functors are theoretically important. In this section, we will present a formal framework which abstracts our examples of interest, and which provides tools for working with and connecting strict and strong morphisms.

In the case of monoidal categories, there is an inclusion $\iota: \mathcal{M n C a t} \rightarrow \widetilde{\mathcal{M n C a t}}$ as well as a forgetful functor $\widehat{\mathcal{M n C a t}} \rightarrow$ Cat. The cases of traced and compact monoidal categories are similar, except there we can factor the forgetful functor through the 2category (or, if one prefers, the $(2,1)$-category) $\mathcal{C} a t \cong$ of categories, functors, and natural isomorphisms. In these examples, we will want to be able to represent strong functors in terms of strict ones, by means of a left adjoint to the inclusion of strict into strong. In Definition A.2.2 we will enumerate properties which are sufficient to prove the existence of this left adjoint, and which are satisfied by all of our motivating examples; see Example A.2.4.

Definition A.2.1. Let $\mathcal{D}_{s}$ and $\tilde{\mathcal{D}}$ be 2-categories and let $\iota: \mathcal{D}_{s} \rightarrow \tilde{\mathcal{D}}$ be a 2-functor that is identity-on-objects, faithful, and locally fully faithful. We say that the triple $\left(\mathcal{D}_{s}, \tilde{\mathcal{D}}, i\right)$ has mapping path objects if $\tilde{\mathcal{D}}$ has mapping path objects as in Definition A.1.5 such that

- for any $f: a \rightarrow b$ in $\tilde{\mathcal{D}}$, the structure morphisms $a \stackrel{\pi_{a}}{\longleftarrow} P(f) \stackrel{\pi_{b}}{\longrightarrow} b$ are in $\mathcal{D}_{s}$, and

- the pair $\left(\pi_{a}, \pi_{b}\right)$ preserves and jointly detects morphisms in $\mathcal{D}_{s}$ in the following sense: for any morphism $\ell: x \rightarrow P(f)$ in $\tilde{\mathcal{D}}$, we have that $\ell$ is in $\mathcal{D}_{s}$ if and only if the compositions $\pi_{a} \circ \ell$ and $\pi_{b} \circ \ell$ are in $\mathcal{D}_{s}$.

We say that the triple $\left(\mathcal{D}_{s}, \tilde{\mathcal{D}}, \iota\right)$ has arrow objects if the analogous conditions hold. 
For the following definition, one may keep in mind the case $\mathcal{D}_{s}=\mathcal{M n C a t}, \tilde{\mathcal{D}}=$ $\widetilde{\mathcal{M n C a t}}$, and $\mathcal{C}=$ Cat. See Example A.2.4 below.

Definition A.2.2. Let $\mathcal{D}_{s}, \tilde{\mathcal{D}}$, and $\mathcal{C}$ be 2-categories, and let $U: \tilde{\mathcal{D}} \rightarrow \mathcal{C}$ and $\iota: \mathcal{D}_{s} \rightarrow \tilde{\mathcal{D}}$ be 2 -functors. We say that the collection $\left(\mathcal{D}_{s}, \tilde{\mathcal{D}}, \mathcal{C}, U, \iota\right)$ admits strong morphism classifiers if it satisfies the following properties:

1. The 2-category $\mathcal{D}_{s}$ has a bijective-on-objects/fully faithful factorization.

2. The functor $\iota$ is identity-on-objects, faithful, and locally fully faithful.

3. The triple $\left(\mathcal{D}_{s}, \tilde{\mathcal{D}}, \iota\right)$ has both arrow objects and mapping path objects (Definition A.2.1).

4. The functor $U \iota: \mathcal{D}_{s} \rightarrow \mathcal{C}$ has a left 2-adjoint $F$.

5. The functor $U \iota$ preserves fully faithful morphisms (equivalently, $F$ preserves bijective-on-objects morphisms).

6. The functor $U$ preserves mapping path objects.

7. The functor $U$ reflects identity 2-cells.

8. The pair $(U \iota, U)$ creates surjective equivalences: given any morphism $f: A \rightarrow B$ in $\mathcal{D}_{s}$ and surjective equivalence $g \dashv U \iota(f)$ in $\mathcal{C}$, there is a unique surjective equivalence $\tilde{g} \dashv \iota f$ in $\tilde{\mathcal{D}}$ such that $U \tilde{g}=g$.

Remark A.2.3. Other than those involving bijective-on-objects or fully faithful morphisms, all of the properties enumerated in Definition A.2.2 (namely, Properties 2, 3, 4, 6, 7, and 8 ) hold whenever $\mathcal{D}_{s}$ is the 2-category of strict algebras and strict morphisms for a 2-monad on $\mathcal{C}$, and $\tilde{\mathcal{D}}$ is the 2-category of strict algebras and pseudo-morphisms. While our main examples can be seen to be algebras for some 2-monad, we have found it easier to isolate just those properties we needed to prove Theorem A.2.5.

This section was strongly inspired by [6] and [15].

Example A.2.4. Suppose that the collection $\left(\mathcal{D}_{s}, \tilde{\mathcal{D}}, \mathcal{C}, l, U\right)$ is defined as in one of the following cases:

- $\mathcal{D}_{s}=\mathcal{M n C a t}, \quad \tilde{\mathcal{D}}=\widetilde{\mathcal{M} \text { Cat }}, \quad \mathcal{C}=$ eat, where $\iota: \mathcal{D}_{s} \rightarrow \tilde{\mathcal{D}}$ is the inclusion and $U: \tilde{\mathcal{D}} \rightarrow \mathcal{C}$ is the forgetful functor;

- $\mathcal{D}_{s}=\mathcal{T}$ rCat $, \quad \tilde{\mathcal{D}}=\widetilde{\mathcal{T} \text { rCat }}, \quad \mathcal{C}=$ Cat $\cong$, where $\iota: \mathcal{D}_{s} \rightarrow \tilde{\mathcal{D}}$ is the inclusion and $U: \tilde{\mathcal{D}} \rightarrow \mathcal{C}$ is the forgetful functor; or

- $\mathcal{D}_{s}=$ epCat $, \quad \tilde{\mathcal{D}}=\widetilde{\text { epCat }}, \quad \mathcal{C}=$ eat $\cong$, where $\iota: \mathcal{D}_{s} \rightarrow \tilde{\mathcal{D}}$ is the inclusion and $U: \tilde{\mathcal{D}} \rightarrow \mathcal{C}$ is the forgetful functor.

We will now show that in each case the collection admits strong morphism classifiers.

Property 1 is proved as Proposition 3.3.6 and the exactness of MnProf, TrProf, and CpProf; see Section 3.3. Property 2 is obvious for $\mathcal{M n C a t}$ and CpCat, and by definition (see Remark 3.1.2) for $\mathcal{T}$ rCat. Property 3 is shown in Remark A.1.7. Property 4 is shown in Corollary A.1.4. Property 5 is a consequence of Proposition 3.3 .6 and Propositions 3.3.1, 3.3.2, and 3.3.5. Property 6 is shown in Remark A.1.7. Property 7 is obvious: if $\alpha: F \rightarrow G$ is a 2-cell in $\mathcal{D}_{s}$ whose underlying natural transformation (in Cat) 
is the identity then it is the identity. It remains to prove Property 8; we first treat the case $\mathcal{D}_{s}=$ MnCat.

Suppose that $F: A \rightarrow B$ is a strict monoidal functor and that there is a surjective equivalence $g \dashv U \iota(F)$ in Cat. Let $f: a \rightarrow b$ denote $U \iota(F)$, so $g: b \rightarrow a$. By Definition A.1.9, we have a 2-cell $\epsilon: g f \cong 1_{a}$ and equalities $1_{b}=f g, \epsilon g=1_{g}$ and $f \epsilon=1_{f}$. A strong functor $G: B \rightarrow A$ with $U G=g$ of course acts the same as $g$ on objects and morphisms. Thus it suffices to give the coherence isomorphisms $\mu: I_{A} \cong G\left(I_{B}\right)$ and $\mu_{x, y}: G(x) \otimes G(y) \stackrel{\cong}{\rightrightarrows} G(x \otimes y)$ for objects $x, y \in B$, which satisfy the required equations. Define $\mu$ to be the composite $I_{A} \stackrel{\epsilon^{-1}}{\longrightarrow} g f\left(I_{A}\right)=g\left(I_{B}\right)$, and define $\mu_{x, y}$ to be the composite

$$
g x \otimes g y \stackrel{\epsilon^{-1}}{\longrightarrow} g f(g x \otimes g y)=g(f g x \otimes f g y)=g(x \otimes y) .
$$

The requisite equations can be checked by direct computation, though they actually follow from a more general theory (doctrinal adjunctions); see [14].

Property 8 holds for the case $\mathcal{D}_{s}=\mathcal{C p}$ Cat because it is a full subcategory of $\mathcal{M}$ nCat. For the case $\mathcal{D}_{s}=\mathcal{T}$ rCat, suppose given a strict traced functor $F: A \rightarrow B$, and let $G: B \rightarrow A$ be the associated monoidal functor constructed above. To see that it is traced, note that $B \stackrel{G}{\rightarrow} A \stackrel{F}{\rightarrow} B$ is the identity, so $G$ is fully faithful, and the result follows from Remark 3.1.3.

Since $\iota$ is identity on objects, we often suppress it for convenience. We draw ordinary arrows $\cdot \rightarrow \cdot$ for morphisms in $\mathcal{D}_{s}$ and snaked arrows, $\cdot \rightsquigarrow \cdot$ for morphisms in $\tilde{\mathcal{D}}$.

Theorem A.2.5. Suppose that $\left(\mathcal{D}_{S}, \tilde{\mathcal{D}}, \mathcal{C}, U, \iota\right)$ admits strong morphism classifiers. Then the functor $\iota$ has a left 2-adjoint $Q: \tilde{D} \rightarrow \mathcal{D}_{s}$. The counit $q_{A}: Q A \rightarrow A$ of this adjunction is given by factoring the counit $\epsilon_{A}$ of the $F \dashv U$ เ adjunction:

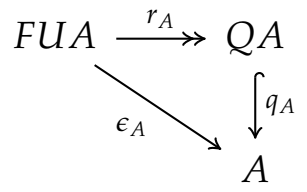

Proof. Define $Q, r$, and $q$ as in (41). We will begin by showing that $q_{A}$ is a surjective equivalence for any $A$, whose inverse $p_{A}: A \rightsquigarrow Q A$ will become the unit of the $Q \dashv \iota$ adjunction. We write $U$ to denote $U \iota$, in a minor abuse of notation. Because $U$ creates surjective equivalences, it suffices to show that $U q_{A}$ is a surjective equivalence. But $q_{A}$ is fully faithful by construction, so $U q_{A}$ is fully faithful, hence by Lemma A.1.10 it suffices to construct a section of $U q_{A}$. We can easily check that $U r_{A} \circ \eta_{U A}$ is such a section:

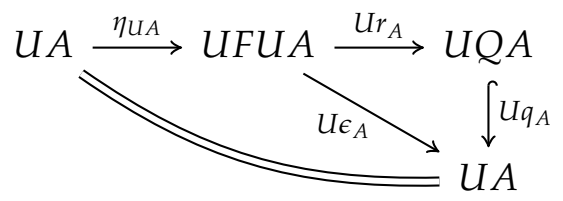

Thus there is a unique surjective equivalence $p_{A} \dashv q_{A}$ in $\tilde{\mathcal{D}}$ such that $U p_{A}=U r_{A} \circ \eta U A$.

We next must show that the morphism $p_{A}: A \rightsquigarrow Q A$ has the following universal property: for any morphism $f: A \rightsquigarrow B$ in $\tilde{\mathcal{D}}$, there is a unique morphism $f^{\prime}: Q A \rightarrow B$ 
in $\mathcal{D}_{s}$ for which $f=f^{\prime} \circ p_{A}$. From this, it follows that $Q$ extends to a 1-functor which is left adjoint to $\iota$. It then follows from Lemma A.1.3 that $Q$ extends to a 2-functor which is left 2-adjoint to $l$, completing the proof of the theorem.

First, given an $f: A \rightsquigarrow B$, define a morphism $\tilde{f}: F U A \rightarrow P(f)$ in $\mathcal{D}_{s}$ as the adjoint of the section $s_{U f}: U A \rightarrow U(P(f))=P(U f)$ defined as in Lemma A.1.11, i.e., $\tilde{f}:=$ $\epsilon_{P(f)} \circ F\left(s_{U f}\right)$. It follows by adjointness that the following diagram in $\mathcal{D}_{s}$ commutes:

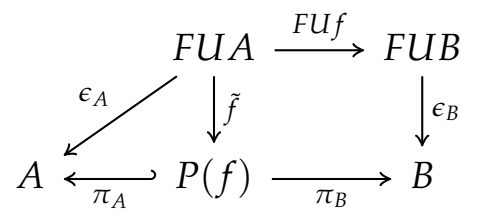

Then by orthogonality there is a unique morphism $\tilde{f}$ in the diagram

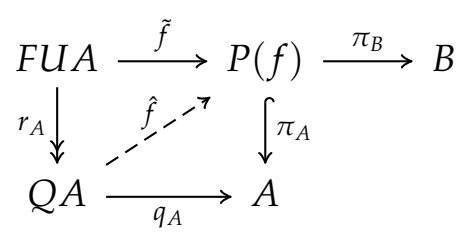

making the square commute, and we define $f^{\prime}:=\pi_{B} \circ \hat{f}$.

We next must check that our definition of $f^{\prime}$ satisfies $f=f^{\prime} \circ p_{A}$. We can construct an isomorphism 2-cell $f \cong f^{\prime} \circ p_{A}$ :

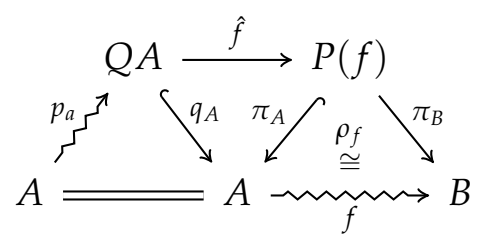

We can check directly that the underlying 2-cell of $\rho_{f} \hat{f} p_{a}$ is the identity on $U f$,

$$
\begin{aligned}
U\left(\rho_{f} \hat{f} p_{a}\right) & =\rho_{U f} U(\hat{f}) U\left(r_{A}\right) \eta_{U A} \\
& =\rho_{U f} U(\tilde{f}) \eta_{U A} \\
& =\rho_{U f} S_{U f} \\
& =1_{U f} .
\end{aligned}
$$

Since $U$ reflects identity 2-cells, it follows that $\rho_{f} \hat{f} q_{A}$ is the identity, $f=f^{\prime} \circ p_{A}$.

Finally, we need to verify that if $f^{\prime \prime}: Q A \rightarrow B$ is any other strict morphism such that $f=f^{\prime \prime} \circ p_{A}$, then $f^{\prime \prime}=f^{\prime}$. We begin by factoring $f^{\prime \prime}=\pi_{B} \circ \hat{f}^{\prime \prime}$ :

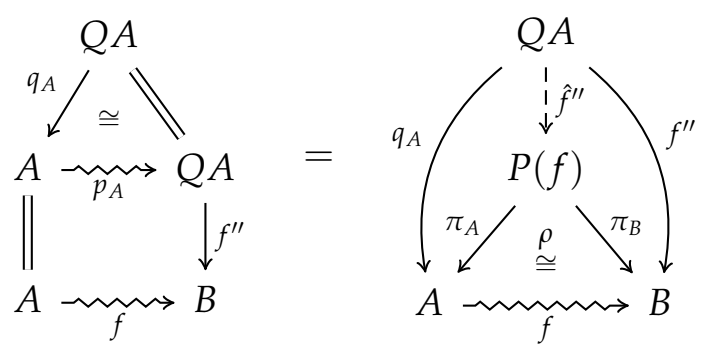


It will then suffice to show that the diagram

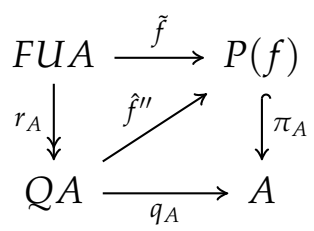

commutes, as then $\hat{f}^{\prime \prime}=\hat{f}$ by orthogonality, and $f^{\prime \prime}=\pi_{B} \hat{f}^{\prime \prime}=\pi_{B} \hat{f}=f^{\prime}$. The lower triangle $\pi_{A} \circ \hat{f}^{\prime \prime}=q_{A}$ follows directly from (42). To show that the upper triangle $\tilde{f}=$ $\hat{f}^{\prime \prime} \circ r_{A}$ commutes, it suffices to check equality of the adjoints $s_{U f}=U\left(\hat{f}^{\prime \prime} \circ r_{A}\right) \circ \eta_{U A}$. We will check this using the universal property of the mapping path object $U P(f)=P(U f)$ by showing that $\rho_{U f} U\left(\hat{f}^{\prime \prime} r_{A}\right) \eta_{U A}=\rho_{U f} \mathcal{S}_{U f}$ :
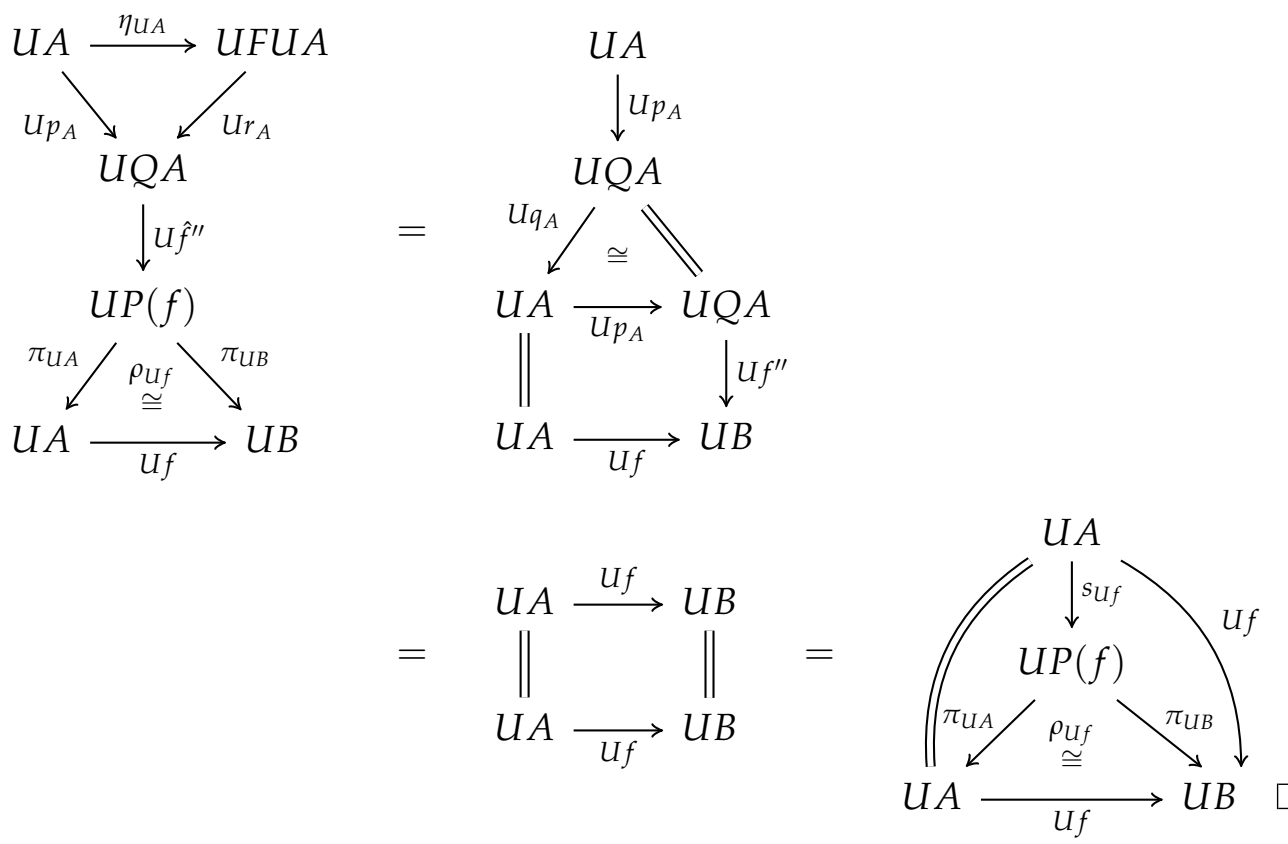

Example A.2.6. Consider the case of Theorem A.2.5 applied to the case where $\mathcal{D}_{s}=$ $\mathcal{M}$ Cat, $\tilde{\mathcal{D}}=\widehat{\mathcal{M} \text { Cat }}$, and $\mathcal{C}=$ Cat. Given any monoidal category $A$, we construct the monoidal category $Q A$ by factoring the counit:

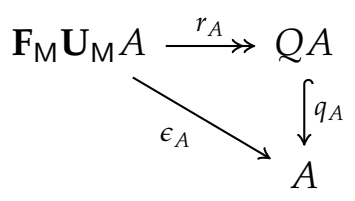

Concretely, this says that the underlying monoid of objects of $Q A$ is the free monoid on $\mathrm{Ob}(A)$, and that given two elements $\left[x_{1}, \ldots, x_{n}\right]$ and $\left[y_{1}, \ldots, y_{m}\right]$ of the free monoid, the hom set is defined

$$
Q A\left(\left[x_{1}, \ldots, x_{n}\right],\left[y_{1}, \ldots, y_{m}\right]\right):=A\left(x_{1} \otimes \cdots \otimes x_{n}, y_{1} \otimes \cdots \otimes y_{m}\right)
$$

Theorem A.2.5 then says that strong monoidal functors out of $A$ are the same as strict monoidal functors out of $Q A$, or more precisely that for any monoidal category $B$ there is an isomorphism of categories $\widehat{\mathcal{M n C a t}}(A, B) \cong \mathcal{M}$ nCat $(Q A, B)$. 
The cases of $\mathcal{T}$ Cat and $\mathcal{E}$ pat are analogous.

\section{A.3 Objectwise-free monoidal, traced, and compact categories}

Our next goal is to show, continuing the assumptions of the Theorem A.2.5, that $\tilde{\mathcal{D}}$ is 2-equivalent to the full subcategory of $\mathcal{D}_{s}$ spanned by those objects which are "objectwise-free". To make this precise, we will further assume we have a 1-category $\mathscr{S}$, together with a fully faithful functor Disc: $\mathscr{S} \rightarrow \mathcal{C}_{0}$ into the underlying category of $\mathcal{C}$ with right adjoint $\mathrm{Ob}$, such that a morphism $f$ in $\mathcal{C}_{0}$ is bo if and only if $\mathrm{Ob} f$ is an isomorphism. The reader may recognize this situation from Proposition 3.5.3. We will write $\mathcal{D}_{\text {FrOb }}$ for the full sub-2-category of $\mathcal{D}_{s}$ spanned by those objects $A$ for which there exists an object $s \in \mathscr{S}$ and a bo morphism $F(\operatorname{Disc}(s)) \rightarrow A$. Then we have that:

Theorem A.3.1. The following composition is a biequivalence of 2-categories:

$$
\mathcal{D}_{\mathrm{FrOb}} \hookrightarrow \mathcal{D}_{S} \stackrel{\iota}{\rightarrow} \tilde{\mathcal{D}}
$$

Proof. We first need to show that $\iota$ induces equivalences of categories $\mathcal{D}_{\mathcal{S}}(A, B) \cong$ $\tilde{\mathcal{D}}(\iota A, \iota B)$ for any $A$ and $B$ which are objectwise-free. In fact, this will hold as long as $A$ is objectwise-free.

If $A \in \mathcal{D}_{s}$ is objectwise-free, then there exists an object $s \in \mathscr{S}$ and a bo morphism $f: F(\operatorname{Disc}(s)) \rightarrow A$ in $\mathcal{D}_{s}$. By the $F \dashv U$ adjunction ${ }^{8}$, there is a unique morphism $\tilde{f}: \operatorname{Disc}(s) \rightarrow U A$ such that $f=\epsilon_{A} \circ F f$. Factoring $\epsilon_{A}=q_{A} \circ r_{A}$ as in Theorem A.2.5, we obtain by orthogonality a unique lift $p_{A}^{\prime} \in \mathcal{D}_{S}$ in the square

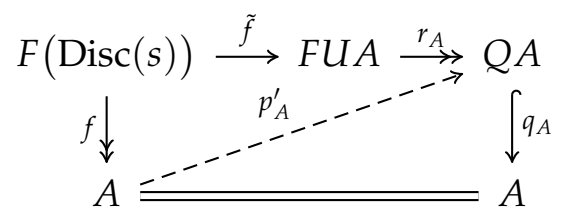

By Lemma A.1.10, it follows that $q_{A}$ is an equivalence in $\mathcal{D}_{s}$. Hence composition with $q_{A}$ induces the left equivalence in

$$
\mathcal{D}_{s}(A, B) \stackrel{\simeq}{\longrightarrow} \mathcal{D}_{s}(Q \iota A, B) \stackrel{\cong}{\longrightarrow} \tilde{\mathcal{D}}(\iota A, \iota B)
$$

and it is easy to check that the composition is precisely $\iota$ on hom categories.

Finally, to prove essential surjectivity, consider an object $A \in \tilde{\mathcal{D}}$. We know that in the factorization

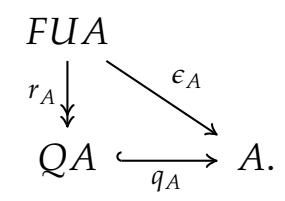

$\iota q_{A}$ is an equivalence in $\tilde{\mathcal{D}}$. We will be done if we can show that $Q A$ is objectwise-free.

\footnotetext{
${ }^{8}$ Note that we continue to commit the abuse of notation writing $U$ for $U \iota$.
} 
Consider the counit $\epsilon_{U A}: \operatorname{Disc}(\mathrm{Ob}(U A)) \rightarrow U A$. Because Disc is fully faithful, it follows that $\mathrm{Ob}\left(\epsilon_{U A}\right)$ is an isomorphism, hence $\epsilon_{U A} \in$ bo, and therefore $F\left(\epsilon_{U A}\right) \in$ bo as well. Thus we can take the composition

$$
F(\operatorname{Disc}(\mathrm{Ob}(U A))) \stackrel{F\left(\epsilon_{U A}\right)}{\longrightarrow} F U A \stackrel{r_{A}}{\longrightarrow} Q A
$$

showing that $Q A$ is objectwise-free.

Corollary A.3.2. The canonical inclusions

$$
\begin{aligned}
\text { MnFrObCat } & \rightarrow \widehat{\mathcal{M} \text { nCat }} \\
\mathcal{T r F r O b C a t} & \rightarrow \widehat{\mathcal{T} \text { rCat }} \\
\text { EpFrObCat } & \rightarrow \widehat{\text { EpCat }}
\end{aligned}
$$

are biequivalences of 2-categories.

Proof. Let $\mathscr{S}=$ Set, and let Disc: $\mathscr{S} \leftrightarrows$ Cat :Ob be the discrete adjunction. Note that a morphism $f$ in Cat is bo if and only if $\mathrm{Ob}(f)$ is an isomorphism. The result follows by Example A.2.4 and Theorem A.3.1.

\section{Bibliography}

[1] S. Abramsky, Retracing some paths in process algebra, CONCUR'96: Concurrency Theory (1996), pp. 1-17.

[2] S. Abramsky, Abstract scalars, loops, and free traced and strongly compact closed categories, Proceedings of CALCO (2005), pp. 1-31.

[3] F. Borceux, Handbook of Categorical Algebra: Volume 1, Basic Category Theory, Cambridge University Press (1994).

[4] F. Borceux, Handbook of Categorical Algebra: Volume 2, Categories and Structures, Cambridge University Press (1994).

[5] F. Borceux, D. Bourn, Mal'cev, protomodular, homological and semi-abelian categories, Mathematics and Its Applications, 566, Kluwer (2004).

[6] J. Bourke, Two-dimensional monadicity, Advances in Mathematics, 252 (2014), pp. 708-747.

[7] T.M. Fiore, N. Gambino, J. Kock, Monads in double categories, J. Pure Appl. Algebra, 215 (2011), no. 6, pp. 1174-1197.

[8] P. Hackney, M. Robertson, On the category of props, Preprint: http://arxiv . org/abs/1207.2773v2 (2012).

[9] M. Hasegawa, S-Y. Katsumata, A note on the biadjunction between 2-categories of traced monoidal categories and tortile monoidal categories, Mathematical Proceedings of the Cambridge Philosophical Society, 148 (2010), no. 1. 
[10] A. Joyal; J. Kock, Feynman graphs, and nerve theorem for compact symmetric multicategories. Electronic Notes in Theoretical Computer Science 270 (2011), 105-113. Preprint: http://arxiv.org/abs/0908.2675 (2009).

[11] A. Joyal; R. Street, Braided tensor categories, Adv. Math. 102 (1993), no. 1, $20 \ddot{i} \varepsilon_{i}-78$.

[12] A. Joyal, R. Street, and D. Verity, Traced monoidal categories, Mathematical Proceedings of the Cambridge Philosophical Society, 119 (1996), no. 3, pp. 447468.

[13] G.M. Kelly, M.L. Laplaza, Coherence for compact closed categories, Journal of Pure and Applied Algebra, 19 (1980), pp. 193-213.

[14] G.M. Kelly, Doctrinal adjunction, Category Seminar (Proc. Sem., Sydney, 1972/1973), Lecture Notes in Math., 420 (1974) Springer, Berlin, pp. 257-280.

[15] S. Lack, Homotopy-theoretic aspects of 2-monads, Journal of Homotopy and Related Structures, 2 (2007), no. 2, pp. 229-260.

[16] T. Leinster, Higher Operads, higher categories, London Mathematical Society Lecture Note Series, 298, Cambridge University Press, Cambridge (2004).

[17] K. Ponto and M. Shulman, Traces in symmetric monoidal categories, Expositiones Mathematicae, 32 (2014), no. 3, pp. 248-273.

[18] D. Rupel and D. I. Spivak, The operad of temporal wiring diagrams: Formalizing a graphical language for discrete-time processes, Preprint: http://arxiv.org/ abs/1307.6894 (2013).

[19] P. Schultz, Regular and exact (virtual) double categories, Preprint: http: //arxiv . org/abs/1505.00712 (2015).

[20] M. Shulman, Framed bicategories and monoidal fibrations, Theory and Applications of Categories, 20 (2008), no. 18, pp. 650-738.

[21] R. J. Wood, Abstract proarrows. I. Cahiers Topologie Géom. Différentielle, 23 (1982), no. 3, pp. 279-290.

[22] R. J. Wood. Proarrows. II. Cahiers Topologie Géom. Différentielle, 26 (1985), no. 2, pp. 135-168. 